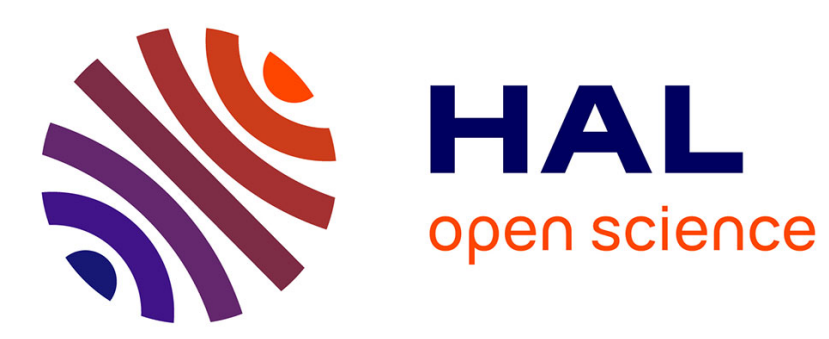

\title{
Paraconsistent OWL and Related Logics
}

Frederick Maier, Yue Ma, Pascal Hitzler

\section{To cite this version:}

Frederick Maier, Yue Ma, Pascal Hitzler. Paraconsistent OWL and Related Logics. Semantic Web Interoperability, Usability, Applicability, 2012. hal-00705876

\section{HAL Id: hal-00705876 https://hal.science/hal-00705876}

Submitted on 8 Jun 2012

HAL is a multi-disciplinary open access archive for the deposit and dissemination of scientific research documents, whether they are published or not. The documents may come from teaching and research institutions in France or abroad, or from public or private research centers.
L'archive ouverte pluridisciplinaire HAL, est destinée au dépôt et à la diffusion de documents scientifiques de niveau recherche, publiés ou non, émanant des établissements d'enseignement et de recherche français ou étrangers, des laboratoires publics ou privés. 


\title{
Paraconsistent OWL and Related Logics
}

\author{
Frederick Maier ${ }^{\natural}$ Yue Ma \\ ${ }^{a}$ Kno.e.sis Center, Wright State University, Dayton, OH, U.S.A. \\ b LIPN - UMR7030, Université Paris 13 - CNRS, France
}

\begin{abstract}
The Web Ontology Language OWL is currently the most prominent formalism for representing ontologies in Semantic Web applications. OWL is based on description logics, and automated reasoners are used to infer knowledge implicitly present in OWL ontologies. However, because typical description logics obey the classical principle of explosion, reasoning over inconsistent ontologies is impossible in OWL. This is so despite the fact that inconsistencies are bound to occur in many realistic cases, e.g., when multiple ontologies are merged or when ontologies are created by machine learning or data mining tools.

In this paper, we present four-valued paraconsistent description logics which can reason over inconsistencies. We focus on logics corresponding to OWL DL and its profiles. We present the logic $\mathcal{S} \mathcal{R} \mathcal{O} \mathcal{I} 4$, showing that it is both sound relative to classical $\mathcal{S R O I} \mathcal{Q}$ and that its embedding into $\mathcal{S R O I Q}$ is consequence preserving. We also examine paraconsistent varieties of $\mathcal{E} \mathcal{L}^{++}$, DL-Lite, and Horn-DLs. The general framework described here has the distinct advantage of allowing classical reasoners to draw sound but nontrivial conclusions from even inconsistent knowledge bases. Truth-value gaps and gluts can also be selectively eliminated from models (by inserting additional axioms into knowledge bases). If gaps but not gluts are eliminated, additional classical conclusions can be drawn without affecting paraconsistency.
\end{abstract}

Keywords: Web Ontology Language, OWL, Description Logic, Paraconsistency, Semantic Web, Complexity, Automated Deduction

\section{Introduction}

The Semantic Web is based fundamentally on the idea that the usefulness of data on the Web can be significantly increased by publishing it together with corresponding metadata, the latter giving a formal and machine processable account of the data's meaning 3132. The Web Ontology Language 30 OWL, now a W3C recommended standard, specifies this metadata using ontologies logical knowledge bases that precisely describe a given domain of interest.

OWL is closely related to description logics (DLs). Specifically, OWL DL corresponds to the description logic $\mathcal{S} \mathcal{R O I} \mathcal{Q}$ [34, while the profiles OWL EL, OWL QL and OWL RL [57] correspond

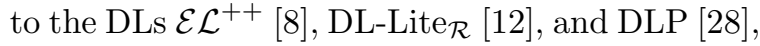
respectively. The formal semantics of OWL, which

${ }^{*}$ Corresponding Author gives meaning to OWL knowledge bases and determines their logical consequences, is based on DL semantics, and the automated tools used to reason over OWL knowledge bases are typically based on algorithms developed for description logics.

Standard description logics are in turn closely related to classical first-order logic [31. In particular, they have a set-theoretic semantics that espouses the law of noncontradiction (no statement can be simultaneously true and false), and the DL account of logical entailment is essentially the same as classical logic's: A set $K$ of statements entails a statement $\mathcal{P}$ if and only if there is no interpretation making all of the members of $K$ true and $\mathcal{P}$ false.

A result of this is that the formal semantics of standard description logics is rendered useless in the presence of contradictory information. Since the statements of an inconsistent knowledge base cannot simultaneously be true, everything is en- 
tailed by them. This is called the principle of explosion.

$$
\text { If } K \models \mathcal{P} \text { and } K \models \neg \mathcal{P} \text {, then } K \models \mathcal{Q} \text { for all } \mathcal{Q} \text {. }
$$

For any statement $\mathcal{P}$ and its negation $\neg \mathcal{P}$, and any set of statements $K$, if both $\mathcal{P}$ and $\neg \mathcal{P}$ are entailed by $K$, then all statements $\mathcal{Q}$ are also entailed by $K$. The principle holds in all standard description logics, and it means that these logics cannot be used to reason over inconsistencies. In Popper's words, "A theory which adds to every information which it asserts also the negation of this information can give us no information at all" 61.

If inconsistencies were rare or could be easily identified, then this problem would be uninteresting. However, real knowledge bases are distributed and multi-authored, or created using inductive methods or by merging other knowledge bases, and it is unreasonable to expect them to be logically consistent all of the time. Furthermore, it is well known that ascertaining consistency in expressive formal systems is quite difficult (sometimes impossible).

And so it is important to develop ways of dealing with inconsistencies. One method of doing this is to maintain the principle of explosion, treating inconsistencies as errors to be repaired (e.g., by rejecting or modifying statements until consistency is restored) 3966]. This approach has the virtue of allowing classical logic to be used, albeit over an altered set of statements. However, in addition to the difficulty of determining whether or not a knowledge base is inconsistent (and also of pinpointing the sources of inconsistencies), the approach has the drawback of deleting or modifying some subset of statements. This necessarily removes valuable information.

An alternative solution is to reject the principle of explosion itself, employing a so-called paraconsistent logic, i.e., a logic in which inconsistencies do not entail everything. Using one, classically sound and informative consequences can be drawn from inconsistent knowledge bases.

The paraconsistency approach is the one taken here. Extending results found in a collection of papers 47748]49|50|51, we present 4-valued paraconsistent semantics for selected DLs and show how knowledge bases under the 4-valued semantics can be embedded (i.e. translated) into corre- sponding knowledge bases under classical DL semantics. The virtue of the approach is that the embedding allows existing classical reasoners to draw inferences according to the new semantics (which, again, are classically sound).

We focus on the logic $\mathcal{S R O I}$ Q4, the paraconsistent variant of $\mathcal{S R O I} \mathcal{Q}$. We prove that $\mathcal{S R O I} 4$ is sound wrt $\mathcal{S R O I} \mathcal{Q}$ and so can be used to cor-

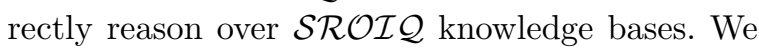
also show that the embedding of $\mathcal{S R O I} 4$ into $\mathcal{S R O I} \mathcal{Q}$ is consequence preserving: a statement is entailed in $\mathcal{S R O I} Q 4$ if and only if its translation is entailed in $\mathcal{S R O I} \mathcal{L}$. Furthermore, we show that by adding axioms of a special type, the law of noncontradiction and the law of the excluded middle can be selectively enforced in $\mathcal{S R O I} Q 4$ knowledge bases. This allows one to draw further classically correct conclusions while at the same time (and with certain caveats) maintaining paraconsistency.

We also apply the paraconsistent framework to several varieties of tractable description logic, including Horn-DLs [43], logics in the DL-Lite family, $\mathcal{E L}^{++}$, and the tractable rule language ELP

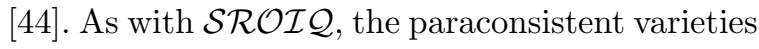
of these can be embedded into their classical counterparts. In order to ensure tractability, however, restrictions must be specified in each case.

Importantly, we also point out a limitation of the paraconsistent framework described here. Specifically, while many DL constructs do not affect paraconsistency one way or another, the interaction of nominals and cardinality restrictions prevents some knowledge bases from having fourvalued models. For these, the principle of explosion still applies.

The remainder of the paper is organized as follows: Section 2 gives a brief overview of multivalued logics and of the difficulties (such as the failure of Modus Ponens) encountered in the 4-valued context. The implication operators (material, internal, and strong) forming the basis for concept inclusion in $\mathcal{S R O I} Q 4$ are also presented. The syntax and semantics for $\mathcal{S R O I} \mathcal{Q} 4$ are defined in Section 3 , and $\mathcal{S R O I Q 4}$ is shown to be sound relative to $\mathcal{S R O I}$. Section 4 discusses removing truth value gaps and gluts from $\mathcal{S R O I} 4$ by adding further axioms. The embedding of $\mathcal{S R O I Q 4}$ into

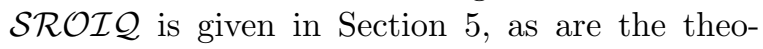
rems asserting that the embedding is consequence preserving. Section 6 shows how the interaction of nominals and cardinality restrictions causes para- 
consistency to break down. Sections 7 and 8 discuss embedding tractable logics into their classical counterparts. It is shown there that tractability is lost if translations are based on strong or material inclusion. A series of empirical tests on OWL ontologies is described in Section 9. Although the experiments are limited and of a tentative nature, their results reinforce the theoretical results of earlier sections: efficient reasoning performance can be obtained if internal inclusion is used, but typically not if material or strong inclusion is used. Section 10 discusses related work.

Proofs for results not reported elsewhere are included in the Appendix.

Acknowledgements: This work is a longer version of materials appearing in 46] and 53, which are in turn based upon earlier research 47/48 49 50, 51. The current work was performed while the first coauthor was a postdoctoral fellow at the Florida Institute for Human and Machine Cognition, and later while at Wright State University. The work was partially supported by the National Science Foundation under award 10172225 "III: Small: TROn - Tractable Reasoning with Ontologies," and partly realized as part of the Quaero Programme, funded by OSEO, the French state agency for innovation.

\section{Multivalued Paraconsistent Logics}

A logic is paraconsistent if the principle of explosion fails in at least one case, that is if there exist statements $\mathcal{P}$ and $\mathcal{Q}$, and a set of statements $K$, such that $K \models \mathcal{P}$ and $K \models \neg \mathcal{P}$ but $K \forall \forall \mathcal{Q}$. Classical logic fails to be paraconsistent because no set entailing $\mathcal{P}$ and $\neg \mathcal{P}$ possesses a model. In the 4 -valued logics upon which $\mathcal{S R O I} 4$ is based, paraconsistency is achieved essentially by redefining what "interpretation" and "model" mean, so that classically inconsistent sets can have models.

We will briefly discuss multivalued paraconsistent logics here. The important properties of multivalued logics can be illustrated with propositional logics over the usual set of connectives: $\neg$ (negation), $\wedge$ (conjunction), $\vee$ (inclusive disjunction), and $\mapsto$ (material implication). As such, we'll restrict ourselves to discussing these.

An $n$-valued logic specifies a set $V$ of truth values, $|V|=n$, and some subset $D \subseteq V$ of desig-

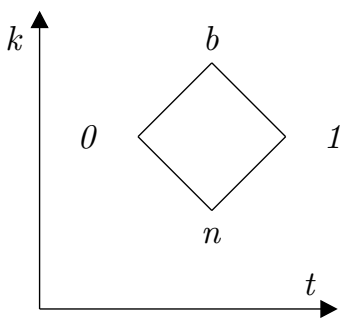

Fig. 1. Belnap's Bilattice

nated values. An $n$-valued interpretation assigns elements of $V$ to each atomic statement, and the values of compound statements are defined using truth-functions. An $n$-valued model of $K$ is an interpretation where each statement of $K$ is given a value from $D$, and $K$ entails $\mathcal{P}$ iff every $n$-valued model of $K$ is also an $n$-valued model of $\mathcal{P}$.

In the context of computing, the use of four truth values $\{n, 0,1, b\}$ can be traced to Belnap 910 , with the four values corresponding to what a computer knows or has been told about $\mathcal{P}$ : nothing $(n)$; that $\mathcal{P}$ is false $(0)$; that $\mathcal{P}$ is true (1); that $\mathcal{P}$ is true and that $\mathcal{P}$ is false $(b)$. Belnap intended the four values to be treated epistemically. They correspond to what the computer has been told about $\mathcal{P}$, rather than denoting an actual truth value.

The semantics for the logical connectives can be given using Belnap's Bilattice (Figure 1). The truth values are partially ordered according to two relations: $\leq_{t}$ (the truth ordering), and $\leq_{k}$ (the knowledge ordering). Both define complete lattices over the set of truth values. The truth-functions for $\wedge$ and $\vee$ are defined as

$$
\begin{aligned}
& -v(\mathcal{P} \wedge \mathcal{Q})=g l b_{\leq_{t}}(v(\mathcal{P}), v(\mathcal{Q})) \\
& -v(\mathcal{P} \vee \mathcal{Q})=l u b_{\leq_{t}}(v(\mathcal{P}), v(\mathcal{Q}))
\end{aligned}
$$

where $g l b_{\leq_{t}}(x, y)$ and $l u b_{\leq_{t}}(x, y)$ refer, respectively, to the greatest lower bound and least upper bound of $x$ and $y$ in the $\leq_{t}$-lattice. Negation inverts the diagram in the $\leq_{t}$-ordering. This semantics yields the characteristic truth tables given in Table 1. where material implication $\mathcal{P} \mapsto \mathcal{Q}$ is defined as $\neg \mathcal{P} \vee \mathcal{Q}$.

The value $b$ is a truth value glut, while $n$ is a truth value gap. If one allows gluts but not gaps, then one gets Graham Priest's 3-valued Logic of Paradox (LP) 62. Allowing gaps but not gluts yields Kleene's strong 3 -valued logic $K_{3}$ 42. If one 
Table 1

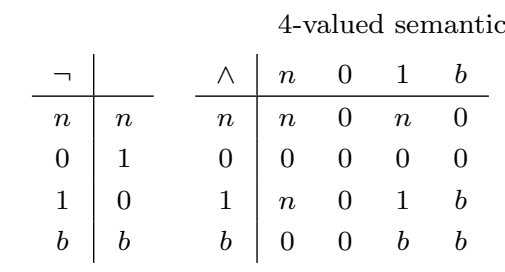

allows neither gaps nor gluts, then one is left with classical logic. Typically, if gluts are allowed in a $\operatorname{logic} L$ and $b$ is designated, then $L$ is paraconsistent. E.g, $L P$ is paraconsistent, while $K_{3}$ is not.

It should be pointed out that, ceteris paribus, all of the consequence relations defined using the multivalued framework possess the following properties.

- Reflexivity: If $\mathcal{P} \in K$, then $K=\mathcal{P}$.

- Monotonicity: If $K \models \mathcal{P}$, then for all $K^{\prime}, K \cup$ $K^{\prime} \models \mathcal{P}$.

- Transitivity: If $K \models \mathcal{P}$ for all $\mathcal{P} \in K^{\prime}$, and $K^{\prime} \models \mathcal{Q}$, then $K \models \mathcal{Q}$.

The same holds for all of the paraconsistent description logics defined in later sections.

Several traditional rules of inference, however, often do not hold in the 4 -valued context. In particular some involving material implication do not hold. Because of this, alternative implication operators (symbolized here using $\rightsquigarrow$ ) have been proposed. Below, $\mathcal{P}$ and $\mathcal{Q}$ are arbitrary statements, and $K$ is an arbitrary set of statements.

- Disjunctive Syllogism: If $K \models \neg \mathcal{P}$ and $K \models$ $\mathcal{P} \vee \mathcal{Q}$, then $K \models \mathcal{Q}$.

- Modus Ponens: If $K \models \mathcal{P} \rightsquigarrow \mathcal{Q}$ and $K \models \mathcal{P}$, then $K \models \mathcal{Q}$.

- Modus Tollens: If $K \models \mathcal{P} \rightsquigarrow \mathcal{Q}$ and $K \models \neg \mathcal{Q}$, then $K \models \neg \mathcal{P}$.

- Identity: $K \models \mathcal{P} \rightsquigarrow \mathcal{P}$.

- Transposition: If $K \models \mathcal{P} \rightsquigarrow \mathcal{Q}$, then $K \models$ $\neg \mathcal{Q} \rightsquigarrow \neg \mathcal{P}$.

- Deduction Theorem: If $K \cup\{\mathcal{P}\} \models \mathcal{Q}$, then $K \models \mathcal{P} \rightsquigarrow \mathcal{Q}$.

- Strong Equivalence: For all truth-value assignments $v,[v(\mathcal{P} \rightsquigarrow \mathcal{Q}) \in\{1, b\}$ and $v(\mathcal{Q} \rightsquigarrow$ $\mathcal{P}) \in\{1, b\}]$ iff $v(\mathcal{P})=v(\mathcal{Q})$.

- Supraclassicality: For all truth-value assignments $v$, if $v(\mathcal{P}) \cup v(\mathcal{Q}) \subseteq\{0,1\}$, then $v(\mathcal{P} \rightsquigarrow$ $\mathcal{Q})=v(\mathcal{P} \mapsto \mathcal{Q})$.

Let $\models_{4}$ indicate the consequence relation defined over the propositional language of $\vee, \wedge, \neg$, and $\mapsto$, and using $V=\{n, 0,1, b\}$ and $D=\{1, b\}$. With the exception of Transposition, none of the above items hold for material implication relative to $\models_{4}$. It is in particular the combined failures of Modus Ponens and the Deduction Theorem that have led to the development of alternative implication operators. $\mathcal{S R O I} \mathcal{Q} 4$ and the other description logics described below use operators defined in the logics of Arieli and Avron 56 6 . The basic operator $(\supset)$ is called internal implication, while strong implication $(\rightarrow)$ is defined in terms of it. Equivalence $(\leftrightarrow)$ is defined in turn using strong implication. For any truth-value assignment $v$, the semantics for the three are given below.

$$
\begin{aligned}
-v(\mathcal{P} \supset \mathcal{Q}) & =1 \text { if } v(\mathcal{P}) \notin\{1, b\} ; \\
v(\mathcal{P} \supset \mathcal{Q}) & =v(\mathcal{Q}) \text { otherwise. } \\
-v(\mathcal{P} \rightarrow \mathcal{Q}) & =v((\mathcal{P} \supset \mathcal{Q}) \wedge(\neg \mathcal{Q} \supset \neg \mathcal{P})) \\
-v(\mathcal{P} \leftrightarrow \mathcal{Q}) & =v((\mathcal{P} \rightarrow \mathcal{Q}) \wedge(\mathcal{Q} \rightarrow \mathcal{P}))
\end{aligned}
$$

The semantics gives rise to the truth tables shown in Table 2, Internal implication satisfies Modus Ponens, the Deduction Theorem, Identity, and Supraclassicality, but not Modus Tollens, Transposition or Strong Equivalence. Strong implication satisfies all of these except (as shown below) the Deduction Theorem.

Example 1. Let $A$ and $\mathcal{P}$ be atomic formulas and $\mathcal{Q}=(A \wedge \neg A)$. Clearly, $\{\mathcal{Q}, \mathcal{P}\} \models_{4} \mathcal{Q}$. However an assignment $v$ on which $v(A)=b$ and $v(\mathcal{P})=1$ shows $\{Q\} \not \nvdash_{4} \mathcal{P} \rightarrow \mathcal{Q}$.

The failure of the Deduction Theorem for strong implication naturally leads one to ask whether there are any operators $\rightsquigarrow$ that satisfy Modus Ponens, Identity, Supraclassicality, Transposition, Modus Tollens, and Strong Equivalence, but also the Deduction Theorem. This is not the case.

Proposition 2. If $\rightsquigarrow$ satisfies Modus Ponens and the Deduction Theorem, then $\rightsquigarrow$ satisfies none of Modus Tollens, Strong Equivalence, or Transposition.

The above proposition holds whether or not Supraclassicality or Identity is satisfied. 
Table 2

Truth tables for internal implication, strong implication, and equivalence.

\begin{tabular}{|c|c|c|c|c|c|c|c|c|c|c|c|c|c|c|}
\hline$\supset$ & $n$ & 0 & 1 & $b$ & $\rightarrow$ & $n$ & 0 & 1 & $b$ & $\leftrightarrow$ & $n$ & 0 & 1 & $b$ \\
\hline$n$ & 1 & 1 & 1 & 1 & $n$ & 1 & $n$ & 1 & $n$ & $n$ & 1 & $n$ & $n$ & $n$ \\
\hline 0 & 1 & 1 & 1 & 1 & 0 & 1 & 1 & 1 & 1 & 0 & $n$ & 1 & 0 & 0 \\
\hline 1 & $n$ & 0 & 1 & $b$ & 1 & $n$ & 0 & 1 & 0 & 1 & $n$ & 0 & 1 & 0 \\
\hline$b$ & $n$ & 0 & 1 & $b$ & $b$ & $n$ & 0 & 1 & $b$ & $b$ & $n$ & 0 & 0 & $b$ \\
\hline
\end{tabular}

Since strong implication satisfies Strong Equivalence, $\mathcal{P} \leftrightarrow \mathcal{Q}$ is a tautology iff the values of $\mathcal{P}$ and $\mathcal{Q}$ coincide in every interpretation. The analogous claim does not hold if equivalence were to be spelled out using $\supset$.

\section{3. $\mathcal{S R O I} \mathcal{I}$ and $\mathcal{S R O I} Q 4$}

The syntax and semantics for classical $\mathcal{S} \mathcal{R O} \mathcal{I}$ are given in Table 3 Below, $N_{C}, N_{R}$, and $N_{I}$ are disjoint sets of atomic concept names, atomic role names, and individual names. Well-formed formulas are created from them, together with the connectives $\neg, \sqcap$, $\sqcup$, etc., and punctuation symbols. A

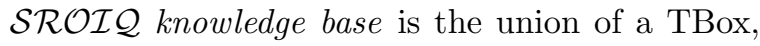
RBox, and ABox, defined below. The original pre-

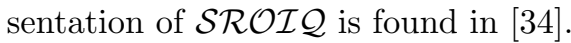

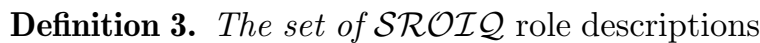
is $N_{R} \cup N_{R}^{-}$, where $N_{R}^{-}=\left\{R^{-} \mid R \in N_{R}\right\}$. R and $R^{-}$are inverses of each other. $N_{R}$ contains a universal role $U$.

Definition 4. $R_{1} \circ \ldots \circ R_{n} \sqsubseteq R$, where $n \geq 1$ and $R, R_{i} \in N_{R} \cup N_{R}^{-}$, is a role-inclusion axiom (RIA). $A$ role-hierarchy is a finite set of RIAs.

Above, an expression such as $R_{1} \circ \ldots \circ R_{n}$ is used to denote a composition of roles. A role $R$ is simple if it: 1) does not appear on the right-hand side of an RIA; 2) is the inverse of a simple role; or 3) appears in the right-hand side of an RIA only if the left-hand side consists entirely of simple roles. Simplicity is needed to ensure decidability of $\mathcal{S R O I Q}$.

Definition 5. Ref(R), $\operatorname{Irr}(R)$, and $\operatorname{Dis}(R, S)$, where $R$ and $S$ are roles other than $U$, are role assertions. A set of role assertions is simple wrt role-hierarchy $H$ if each assertion $\operatorname{Irr}(R)$ and $\operatorname{Dis}(R, S)$ uses only roles that are simple wrt $H$.
Ref, Irr, and Dis are used to specify that a role is reflexive, irreflexive, or disjoint with another role. Sym and Trans may be used to express symmetry and transitivity, but since they can be encoded via other means, we will not discuss them.

Decidability also requires the role hierarchy to be regular.

Definition 6. A strict partial order $\prec$ on $N_{R} \cup N_{R}^{-}$ is a regular order iff the following holds for all roles $R$ and $S: S \prec R$ iff $S^{-} \prec R$.

Definition 7. Let $\prec$ be a regular order on roles. An $R I A w \sqsubseteq R$ is $\prec$-regular iff $R \in R_{N}$ and $w$ has one of the below forms.

1. $R \circ R$

2. $R^{-}$

3. $S_{1} \circ \ldots \circ S_{n}$, where each $S_{i} \prec R$

4. $R \circ S_{1} \circ \ldots \circ S_{n}$, where each $S_{i} \prec R$

5. $S_{1} \circ \ldots \circ S_{n} \circ R$, where each $S_{i} \prec R$

$A$ role hierarchy $H$ is regular if there exists a regular order $\prec$ such that each RIA in $H$ is $\prec$-regular.

Definition 8. An RBox is a finite, regular role hierarchy $H$ together with a finite set of role assertions simple wrt $H$.

Definition 9. If $a_{1}, \ldots, a_{n}$ are in $N_{I}$, then $\left\{a_{1}, \ldots\right.$, $\left.a_{n}\right\}$ is a nominal. $N_{o}$ is the set of nominals.

Definition 10. The set of $\mathcal{S R O I Q}$-concept descriptions is the smallest set such that:

1. $\perp$, $\top$, each $C \in N_{C}$, and each $o \in N_{o}$ is a concept description.

2. If $C$ is a concept description, then $\neg C$ is a concept description.

3. If $C$ and $D$ are concept descriptions, $R$ is a role description, $S$ is a simple role description, and $n$ is a nonnegative integer, then the following are all concept descriptions:

$(C \sqcap D), \quad(C \sqcup D), \quad \exists R . C, \quad \forall R . C$, $\leq n S . C, \quad \geq n$ S.C. $\quad \exists$ S.Self. 
Table 3

Syntax and semantics of $\mathcal{S R O I Q}$.

\begin{tabular}{|c|c|c|}
\hline Concept & Syntax & $\mathcal{S R O I} \mathcal{Q}$ Semantics \\
\hline $\begin{array}{l}\text { atomic } \\
\text { concept }\end{array}$ & $C \in N_{C}$ & $C^{\mathcal{I}} \subseteq \Delta^{\mathcal{I}}$ \\
\hline individual & $a \in N_{I}$ & $a^{\mathcal{I}} \in \Delta^{\mathcal{I}}$ \\
\hline nominal & $\left\{a_{1}, \ldots, a_{n}\right\}, a_{i} \in N_{I}$ & $\left\{a_{1}^{\mathcal{I}}, \ldots, a_{n}^{\mathcal{I}}\right\}$ \\
\hline role & $R \in N_{R}$ & $R^{\mathcal{I}} \subseteq \Delta^{\mathcal{I}} \times \Delta^{\mathcal{I}}$ \\
\hline inverse role & $R^{-}, R \in N_{R}$ & $R^{-\mathcal{I}}=\left\{(y, x) \mid(x, y) \in R^{\mathcal{I}}\right\}$ \\
\hline universal role & $U$ & $U^{\mathcal{I}}=\Delta^{\mathcal{I}} \times \Delta^{\mathcal{I}}$ \\
\hline $\begin{array}{c}\text { role } \\
\text { composition }\end{array}$ & $R_{1} \circ \ldots \circ R_{n}$ & $\begin{aligned}\left\{(x, z) \mid\left(x, y_{1}\right)\right. & \in R_{1}^{\mathcal{I}} \wedge\left(y_{1}, y_{2}\right) \in R_{2}^{\mathcal{I}} \wedge \ldots \\
\ldots & \left.\wedge\left(y_{n}, z\right) \in R_{n}^{\mathcal{I}}\right\}\end{aligned}$ \\
\hline top & $T$ & $\top^{\mathcal{I}}=\Delta^{\mathcal{I}}$ \\
\hline bottom & $\perp$ & $\perp^{\mathcal{I}}=\varnothing$ \\
\hline negation & $\neg C$ & $(\neg C)^{\mathcal{I}}=\Delta^{\mathcal{I}} / C^{\mathcal{I}}$ \\
\hline conjunction & $C_{1} \sqcap C_{2}$ & $\left(C_{1} \sqcap C_{2}\right)^{\mathcal{I}}=C_{1}^{\mathcal{I}} \cap C_{2}^{\mathcal{I}}$ \\
\hline disjunction & $C_{1} \sqcup C_{2}$ & $\left(C_{1} \sqcup C_{2}\right)^{\mathcal{I}}=C_{1}^{\mathcal{I}} \cup C_{2}^{\mathcal{I}}$ \\
\hline $\begin{array}{c}\text { exists } \\
\text { restriction }\end{array}$ & $\exists R . C$ & $\left\{x \mid(\exists y)\left[(x, y) \in R^{\mathcal{I}} \wedge y \in C^{\mathcal{I}}\right]\right\}$ \\
\hline $\begin{array}{c}\text { value } \\
\text { restriction }\end{array}$ & $\forall R . C$ & $\left\{x \mid(\forall y)\left(\left[(x, y) \in R^{\mathcal{I}} \mapsto y \in C^{\mathcal{I}}\right]\right\}\right.$ \\
\hline $\begin{array}{c}\text { self } \\
\text { restriction }\end{array}$ & $\exists R . S e l f$ & $\left\{x \mid(x, x) \in R^{\mathcal{I}}\right\}$ \\
\hline $\begin{array}{l}\text { atmost } \\
\text { restriction }\end{array}$ & $\leq n R . C$ & $\left\{x \mid \sharp\left\{y \mid(x, y) \in R^{\mathcal{I}} \wedge y \in C^{\mathcal{I}}\right\} \leq n\right\}$ \\
\hline $\begin{array}{l}\text { atleast } \\
\text { restriction }\end{array}$ & $\geq n R . C$ & $\left\{x \mid \sharp\left\{y \mid(x, y) \in R^{\mathcal{I}} \wedge y \in C^{\mathcal{I}}\right\} \geq n\right\}$ \\
\hline
\end{tabular}

\begin{tabular}{|c|c|c|}
\hline Axiom & Syntax & $\mathcal{S} \mathcal{O} \mathcal{I} \mathcal{I}$ Semantics \\
\hline $\begin{array}{c}\text { concept } \\
\text { inclusion }\end{array}$ & $C_{1} \sqsubseteq C_{2}$ & $C_{1}^{\mathcal{I}} \subseteq C_{2}^{\mathcal{I}}$ \\
\hline $\begin{array}{c}\text { role } \\
\text { inclusion }\end{array}$ & $R_{1} \circ \ldots \circ R_{n} \sqsubseteq R_{n+1}$ & $\left(R_{1} \circ \ldots \circ R_{n}\right)^{\mathcal{I}} \subseteq R_{n+1}^{\mathcal{I}}$ \\
\hline reflexivity & $\operatorname{Ref}(R)$ & $\left\{(x, x) \mid x \in \Delta^{\mathcal{I}}\right\} \subseteq R^{\mathcal{I}}$ \\
\hline irreflexivity & $\operatorname{Irr}(R)$ & $\left\{(x, x) \mid x \in \Delta^{\mathcal{I}}\right\} \cap R^{\mathcal{I}}=\varnothing$ \\
\hline disjointness & $\operatorname{Dis}(R, S)$ & $S^{\mathcal{I}} \cap R^{\mathcal{I}}=\varnothing$ \\
\hline $\begin{array}{c}\text { class } \\
\text { assertion }\end{array}$ & $C(a)$ & $a^{\mathcal{I}} \in C^{\mathcal{I}}$ \\
\hline $\begin{array}{c}\text { inequality } \\
\text { assertion }\end{array}$ & $a \neq b$ & $\left(a^{\mathcal{I}}, b^{\mathcal{I}}\right) \in R^{\mathcal{I}}$ \\
\hline $\begin{array}{c}\text { role (instance } \\
\text { assertion }\end{array}$ & $R(a, b)$ & $\left(a^{\mathcal{I}}, b^{\mathcal{I}}\right) \notin R^{\mathcal{I}}$ \\
\hline $\begin{array}{c}\text { negative role } \\
\text { assertion }\end{array}$ & $\neg R(a, b)$ & \\
\hline
\end{tabular}


Definition 11. If $C$ and $D$ are concept descriptions, then $C \sqsubseteq D$ is a general concept inclusion (GCI) axiom. A TBox is a finite set of GCIs.

Definition 12. If $C$ is a concept description, $a, b \in$ $N_{I}, R, S \in N_{R} \cup N_{R}^{-}$, and $S$ is a simple role description, then $C(a), R(a, b), \neg S(a, b)$, and $a \neq b$, are individual assertions. $A \mathcal{S R O I \mathcal { Q }} \mathrm{ABox}$ is a set of individual assertions.

We will call all GCIs, RIAs, role assertions, and individual assertions, axioms.

The semantics of $\mathcal{S R O I Q}$ is classical. A 2valued interpretation (or 2-interpretation) is a tuple $\left\langle\Delta^{\mathcal{I}},{ }^{\mathcal{I}}\right\rangle$, where $\Delta^{\mathcal{I}}$ is a nonempty set (the universe of discourse), and.$^{\mathcal{I}}$ is a valuation function defined inductively as shown in Table 3 . A 2interpretation $\mathcal{I} 2$-satisfies (is a 2-model of ) an axiom $A$ if the corresponding condition of $\mathcal{S R O I \mathcal { Q }}$ in Table 3 is met. $\mathcal{I} 2$-satisfies (is a 2-model of) a knowledge base $K B$ iff it 2-satisfies each axiom in $K B . K B$ is 2-satisfiable (2-unsatisfiable) iff it has (does not have) a 2-model. $K B$ entails an axiom $A$ wrt $\mathcal{S R O I \mathcal { Q }}(K B=\mathcal{S R O I \mathcal { Q }} A)$ iff every 2-model of $K B$ is a 2-model of $A$.

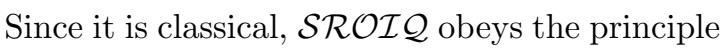
of explosion.

Example 13. Let $K B$ be a $\mathcal{S R O I} \mathcal{Q}$ knowledge base containing the following axioms:

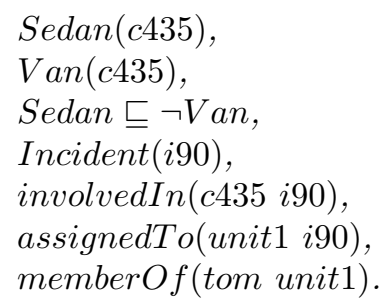

The knowledge base is intended to describe an accident involving a vehicle, the response team assigned to the accident, and the members of the team. The first three axioms are inconsistent, however, and because of this, unintuitive consequences such as memberOf(c435 unit 1$)$ and Incident(tom) are entailed according to the clas-

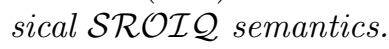

To create the 4-valued logic $\mathcal{S R O I}$ Q4, we assign to each concept description both a positive extension $P$ and a negative extension (anti-extension) $N$. $C(a)$ can thereby take on one of Belnap's four values.

$$
\begin{array}{ll}
1: & a^{\mathcal{I}} \in P, a^{\mathcal{I}} \notin N . \\
0: & a^{\mathcal{I} \notin P,} a^{\mathcal{I} \in N .} \\
n: & a^{\mathcal{I}} \notin P, a^{\mathcal{I}} \notin N . \\
b: & a^{\mathcal{I}} \in P, a^{\mathcal{I}} \in N .
\end{array}
$$

Roles are given a 4 -valued semantics as well (in earlier papers [46, we at times treated them classically). Specifically, $R^{\mathcal{I}}$ is $\langle P, N\rangle$, where $P$ and $N$ are subsets of $\Delta^{\mathcal{I}} \times \Delta^{\mathcal{I}}$. The reason for this treatment is to maintain paraconsistency while allowing Irr, Dis, Ref, $\exists$ R.Self , and negative role assertions to be used in knowledge bases.

The syntax for $\mathcal{S R O I} Q 4$ differs from $\mathcal{S R O I} \mathcal{Q}$ in that it allows three types of inclusion axiom, based on the operators of Arieli and Avron's logic.

$$
\begin{aligned}
& C_{1} \mapsto C_{2} \\
& C_{1} \sqsubset C_{2} \\
& C_{1} \rightarrow C_{2}
\end{aligned}
$$

Strictly speaking, axioms using the new inclusion operators are not defined in classical $\mathcal{S R O I} \mathcal{L}$. However, we choose to extend the $\mathcal{S R O I Q}$ syntax and semantics, stipulating that $(C \mapsto D)$, $(C \sqsubset D)$, and $(C \rightarrow D)$ have the same semantics in $\mathcal{S R O I \mathcal { Q }}$ as $(C \sqsubseteq D)$. We also stipulate that $(C \sqsubseteq D)$ is to be read as $(C \sqsubset D)$ in $\mathcal{S R O I}$ Q4. This allows a common language $\mathcal{L}$ for both $\mathcal{S R O I} \mathcal{Q}$ and $\mathcal{S R O I} 44$ knowledge bases and simplifies some of the proofs later in the paper.

If a knowledge base makes use of only $\sqsubseteq$, then

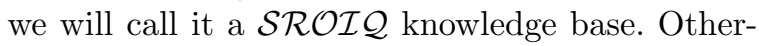
wise, it is a $\mathcal{S R O I} 4$ knowledge base. It should be noted that $\mathcal{S R O I Q}$ encompasses several other description logics discussed in the literature. E.g, $\mathcal{A L C}$ is the logic defined using only $N_{C}, N_{R}, N_{I}$,

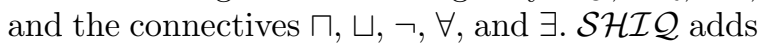
qualified cardinality restrictions ${ }^{1}$ As this is so, we can also speak of $\mathcal{A L C} 4$ and $\mathcal{S H \mathcal { I }} 4$ knowledge bases. The paraconsistent framework defined here for $\mathcal{S R O I} \mathcal{Q}$ applies equally well to them.

The semantics of $\mathcal{S R O I Q 4}$ parallels that of its classical counterpart. A 4-valued interpretation (4-interpretation) is a tuple $\left\langle\Delta^{\mathcal{I}},{ }^{\mathcal{I}}\right\rangle$, where $\Delta^{\mathcal{I}}$ is nonempty and ${ }^{\mathcal{I}}$ is a valuation function defined inductively as shown in Table4. We will also make use of the following notation: If $C$ is a concept and $\mathcal{I}$ is a 4-interpretation such that $C^{\mathcal{I}}=\langle P, N\rangle$,

${ }^{1} \mathcal{S H \mathcal { C }}$ also allows an assertion of the form $\operatorname{Trans}(R)$. However, this can be simulated in $\mathcal{S} \mathcal{R O} \mathcal{I} \mathcal{Q}$. 
Table 4

Syntax and semantics of $\mathcal{S R O I Q 4}$.

\begin{tabular}{|c|c|}
\hline Syntax & $\mathcal{S R O I} Q 4$ Semantics \\
\hline$C \in N_{C}$ & $C^{\mathcal{I}}=\langle P, N\rangle$, where $P, N \subseteq \Delta^{\mathcal{I}}$ \\
\hline$a \in N_{I}$ & $a^{\mathcal{I}} \in \Delta^{\mathcal{I}}$ \\
\hline$\left\{a_{1}, \ldots, a_{n}\right\}, a_{i} \in N_{I}$ & $\left\langle\left\{a_{1}^{\mathcal{I}}, \ldots, a_{n}^{\mathcal{I}}\right\}, N\right\rangle$, where $N \subseteq \Delta^{\mathcal{I}}$ \\
\hline$R \in N_{R}$ & $R^{\mathcal{I}}=\langle P, N\rangle$, where $P, N \subseteq \Delta^{\mathcal{I}} \times \Delta^{\mathcal{I}}$ \\
\hline$R^{-}, R \in N_{R}$ & $\begin{array}{c}R^{-\mathcal{I}}=\left\langle P^{-}, N^{-}\right\rangle, \text {where } R^{\mathcal{I}}=\langle P, N\rangle, \\
P^{-}=\{(b, a) \mid(a, b) \in P\}, N^{-}=\{(b, a) \mid(a, b) \in N\}\end{array}$ \\
\hline$U$ & $U^{\mathcal{I}}=\left\langle\Delta^{\mathcal{I}} \times \Delta^{\mathcal{I}}, \varnothing\right\rangle$ \\
\hline$R_{1} \circ \ldots \circ R_{n}$ & $\begin{aligned} &\left\langle\left\{(x, z) \mid\left(\exists y_{1 \ldots n}\right)\left[\left(x, y_{1}\right) \in p^{+}\left(R_{1}^{\mathcal{I}}\right) \wedge \ldots \wedge\left(y_{n}, z\right) \in p^{+}\left(R_{n}^{\mathcal{I}}\right]\right\}\right)\right. \\
&\left.\left\{(x, z) \mid\left(\forall y_{1 \ldots n}\right)\left[\left(x, y_{1}\right) \in p^{-}\left(R_{1}^{\mathcal{I}}\right) \vee \ldots \vee\left(y_{n}, z\right) \in p^{-}\left(R_{n}^{\mathcal{I}}\right]\right\}\right)\right\rangle\end{aligned}$ \\
\hline$T$ & $\top^{\mathcal{I}}=\left\langle\Delta^{\mathcal{I}}, \varnothing\right\rangle$ \\
\hline$\perp$ & $\perp^{\mathcal{I}}=\left\langle\varnothing, \Delta^{\mathcal{I}}\right\rangle$ \\
\hline$\neg C$ & $(\neg C)^{\mathcal{I}}=\langle N, P\rangle$, where $C^{\mathcal{I}}=\langle P, N\rangle$ \\
\hline$C_{1} \sqcap C_{2}$ & $\left(C_{1} \sqcap C_{2}\right)^{\mathcal{I}}=\left\langle P_{1} \cap P_{2}, N_{1} \cup N_{2}\right\rangle$, where $C_{i}^{\mathcal{I}}=\left\langle P_{i}, N_{i}\right\rangle$ \\
\hline$C_{1} \sqcup C_{2}$ & $\left(C_{1} \sqcup C_{2}\right)^{\mathcal{I}}=\left\langle P_{1} \cup P_{2}, N_{1} \cap N_{2}\right\rangle$, where $C_{i}^{\mathcal{I}}=\left\langle P_{i}, N_{i}\right\rangle$ \\
\hline$\exists R . C$ & $\begin{array}{l}\left\langle\left\{x \mid(\exists y)\left[(x, y) \in p^{+}\left(R^{\mathcal{I}}\right) \wedge y \in p^{+}\left(C^{\mathcal{I}}\right)\right]\right\},\right. \\
\left.\left\{x \mid(\forall y)\left[(x, y) \in p^{+}\left(R^{\mathcal{I}}\right) \mapsto y \in p^{-}\left(C^{\mathcal{I}}\right)\right]\right\}\right\rangle\end{array}$ \\
\hline$\forall R . C$ & $\begin{array}{l}\left\langle\left\{x \mid(\forall y)\left(\left[(x, y) \in p^{+}\left(R^{\mathcal{I}}\right) \mapsto y \in p^{+}\left(C^{\mathcal{I}}\right)\right]\right\},\right.\right. \\
\left.\left\{x \mid(\exists y)\left[(x, y) \in p^{+}\left(R^{\mathcal{I}}\right) \wedge y \in p^{-}\left(C^{\mathcal{I}}\right)\right]\right\}\right\rangle\end{array}$ \\
\hline$\exists R . S e l f$ & $\left\langle\left\{x \mid(x, x) \in p^{+}\left(R^{\mathcal{I}}\right)\right\}, N\right\rangle, N \subseteq \Delta^{\mathcal{I}}$ \\
\hline$\leq n R \cdot C$ & $\begin{array}{l}\left\langle\left\{x \mid \sharp\left\{y \mid(x, y) \in p^{+}\left(R^{\mathcal{I}}\right) \wedge y \notin p^{-}\left(C^{\mathcal{I}}\right)\right\} \leq n\right\},\right. \\
\left.\left\{x \mid \sharp\left\{y \mid(x, y) \in p^{+}\left(R^{\mathcal{I}}\right) \wedge y \in p^{+}\left(C^{\mathcal{I}}\right)\right\}>n\right\}\right\rangle\end{array}$ \\
\hline$\geq n R \cdot C$ & $\begin{array}{l}\left\langle\left\{x \mid \sharp\left\{y \mid(x, y) \in p^{+}\left(R^{\mathcal{I}}\right) \wedge y \in p^{+}\left(C^{\mathcal{I}}\right)\right\} \geq n\right\},\right. \\
\left.\left\{x \mid \sharp\left\{y \mid(x, y) \in p^{+}\left(R^{\mathcal{I}}\right) \wedge y \notin p^{-}\left(C^{\mathcal{I}}\right)\right\}<n\right\}\right\rangle\end{array}$ \\
\hline
\end{tabular}

\begin{tabular}{|c|c|}
\hline Syntax & $\mathcal{S R O I} Q 4$ Semantics \\
\hline$C_{1} \mapsto C_{2}$ & $\left(\Delta^{\mathcal{I}}-p^{-}\left(C_{1}^{\mathcal{I}}\right)\right) \subseteq p^{+}\left(C_{2}^{\mathcal{I}}\right)$ \\
\hline$C_{1} \sqsubset C_{2}$ & $p^{+}\left(C_{1}^{\mathcal{I}}\right) \subseteq p^{+}\left(C_{2}^{\mathcal{I}}\right)$ \\
\hline$C_{1} \rightarrow C_{2}$ & $p^{+}\left(C_{1}^{\mathcal{I}}\right) \subseteq p^{+}\left(C_{2}^{\mathcal{I}}\right)$ and $p^{-}\left(C_{2}^{\mathcal{I}}\right) \subseteq p^{-}\left(C_{1}^{\mathcal{I}}\right)$ \\
\hline$R_{1} \circ \ldots \circ R_{n} \sqsubseteq R_{n+1}$ & $p^{+}\left(\left(R_{1} \circ \ldots \circ R_{n}\right)^{\mathcal{I}}\right) \subseteq p^{+}\left(R_{n+1}^{\mathcal{I}}\right)$ \\
\hline $\operatorname{Ref}(R)$ & $\left\{(x, x) \mid x \in \Delta^{\mathcal{I}}\right\} \subseteq p^{+}\left(R^{\mathcal{I}}\right)$ \\
\hline $\operatorname{Irr}(R)$ & $\left\{(x, x) \mid x \in \Delta^{\mathcal{I}}\right\} \subseteq p^{-}\left(R^{\mathcal{I}}\right)$ \\
\hline $\operatorname{Dis}(R, S)$ & $p^{+}\left(R^{\mathcal{I}}\right) \subseteq p^{-}\left(S^{\mathcal{I}}\right)$ and $p^{+}\left(S^{\mathcal{I}}\right) \subseteq p^{-}\left(R^{\mathcal{I}}\right)$ \\
\hline$C(a)$ & $a^{\mathcal{I}} \in p^{+}\left(C^{\mathcal{I}}\right)$ \\
\hline$a \neq b$ & $a^{\mathcal{I}} \neq b^{\mathcal{I}}$ \\
\hline$R(a, b)$ & $\left(a^{\mathcal{I}}, b^{\mathcal{I}}\right) \in p^{+}\left(R^{\mathcal{I}}\right)$ \\
\hline$\neg R(a, b)$ & $\left(a^{\mathcal{I}}, b^{\mathcal{I}}\right) \in p^{-}\left(R^{\mathcal{I}}\right)$ \\
\hline
\end{tabular}


then $p^{+}\left(C^{\mathcal{I}}\right)=_{\text {def }} P$ and $p^{-}\left(C^{\mathcal{I}}\right)==_{\text {def }} N$. A 4interpretation 4-satisfies (is a 4-model of) an axiom $A$ if the corresponding condition of $\mathcal{S R O I Q 4}$ in Table 4 is met. $\mathcal{I} 4$-satisfies a knowledge base $K B$ iff it 4-satisfies each axiom in $K B$. $K B$ is 4-satisfiable (4-unsatisfiable) iff it has (does not have) a 4-model. $K B$ entails an axiom $A$ wrt $\mathcal{S R O I} 4\left(K B \models_{\mathcal{S R O I} Q} 4\right)$ iff every 4 -model of $K B$ is a 4 -model of $A$.

Example 14. The following interpretation is a 4model of the knowledge base in Example 13.

$\Delta^{\mathcal{I}}=\{c 435$, i90, tom, unit 1$\}$

$c 435^{\mathcal{I}}=c 435$

$i 90^{\mathcal{I}}=i 90$

tom $^{\mathcal{I}}=$ tom

unit $1^{\mathcal{I}}=$ unit 1

$\operatorname{Sedan}^{\mathcal{I}}=\operatorname{Van}^{\mathcal{I}}=\left\langle\{c 435\}, \Delta^{\mathcal{I}}\right\rangle$

Incident ${ }^{\mathcal{I}}=\left\langle\{i 90\}, \Delta^{\mathcal{I}}-\{i 90\}\right\rangle$

involvedIn $^{\mathcal{I}}=\left\langle\{(c 435, i 90)\},\left(\Delta^{\mathcal{I}}\right)^{2}-\{(c 435, i 90)\}\right\rangle$

assignedTo $o^{\mathcal{I}}=\left\langle\{(\right.$ unit $1, i 90)\},\left(\Delta^{\mathcal{I}}\right)^{2}-\{($ unit $\left.1, i 90)\}\right\rangle$

memberOf $f^{\mathcal{I}}=\left\langle\{(\right.$ tom, unit 1$)\},\left(\Delta^{\mathcal{I}}\right)^{2}-\{($ tom, unit 1$\left.)\}\right\rangle$

The interpretation in Example 14 is a 4-model regardless of whether the single inclusion axiom of the knowledge base is read as material inclusion, internal inclusion, or strong inclusion. The interpretation is not a 4-model of the assertions Incident(tom) and memberOf(c435, unit 1$)$, however, and so the knowledge base does not entail them according to $\mathcal{S R O I} 4$.

It should be pointed out that internal inclusion $\sqsubset$ approximates $\rightarrow$, in the sense that the consequences obtainable using $\sqsubset$ are a subset of those obtainable using $\rightarrow$.

Proposition 15. Let $K B$ be a $\mathcal{S R O I} \mathcal{Q} 4$ knowledge base, $A$ a $\mathcal{S R O I} Q 4$ axiom, and $K B^{\prime}$ the knowledge base obtained by replacing one or more occurrences of $\sqsubset$ with $\rightarrow$. If $K B \models A$, then $K B^{\prime} \models A$.

In contrast, material inclusion $\mapsto$ does not approximate $\sqsubset$ in a similar way.

Example 16. In general, $\{C \mapsto D\} \models C \mapsto D$ holds in $\mathcal{S R O I Q 4}$ but $\{C \sqsubset D\} \not \models C \mapsto D$ does not. This can be seen by modifying the interpretation in Example 14 so that Sedan ${ }^{\mathcal{I}}$ and Van $^{\mathcal{I}}$ are defined to be $\langle\{c 435\},\{c 435\}\rangle$. Under the modified interpretation, Sedan $\rightarrow \neg$ Van is satisfied but Sedan $\mapsto \neg V$ an is not.
In $\mathcal{S R O I} Q 4$, inequality assertions $a \neq b$ have a classical semantics: $a \neq b$ is true if and only if $a^{\mathcal{I}} \neq b^{\mathcal{I}}$, and it is false otherwise. In other words, we have not given (in)equality a paraconsistent semantics. This is done to facilitate embedding $\mathcal{S R O I} 44$ into classical $\mathcal{S R O I} \mathcal{Q}$ so that classical tools can be used to reason paraconsistently. However, the use of classical semantics here entails the existence of $\mathcal{S R O I} \mathcal{Q} 4$ knowledge bases that do not have 4 -valued models. This is discussed in subsequent sections.

As in the classical setting, several equivalences involving negation hold in $\mathcal{S R O I Q 4}$.

Proposition 17. For any $\mathcal{S R O I} Q 4$ concepts $C, D$ and 4-interpretation $\mathcal{I}$ the following hold.
1. $(\neg \top)^{\mathcal{I}}=\perp^{\mathcal{I}}$
2. $(\neg \perp)^{\mathcal{I}}=\top^{\mathcal{I}}$
3. $(\neg \neg C)^{\mathcal{I}}=C^{\mathcal{I}}$
4. $(\neg(C \sqcap D))^{\mathcal{I}}=(\neg C \sqcup \neg D)^{\mathcal{I}}$
5. $(\neg(C \sqcup D))^{\mathcal{I}}=(\neg C \sqcap \neg D)^{\mathcal{I}}$
6. $(\neg \exists R \cdot C)^{\mathcal{I}}=(\forall R . \neg C)^{\mathcal{I}}$
7. $(\neg \forall R . C)^{\mathcal{I}}=(\exists R . \neg C)^{\mathcal{I}}$
8. $(\neg \leq n R . C)^{\mathcal{I}}=(\geq n+1 R \cdot C)^{\mathcal{I}}$
9. $(\neg \geq n R . C)^{\mathcal{I}}=(\leq n-1 R \cdot C)^{\mathcal{I}}$

In the classical DL setting, concept $C$ is $s u b$ sumed by concept $D$ if and only if $C^{\mathcal{I}} \subseteq D^{\mathcal{I}}$ for each classical interpretation $\mathcal{I}$, and $C$ and $D$ are equivalent if and only if $C^{\mathcal{I}}=D^{\mathcal{I}}$. These are easily related to satisfiability of GCIs. Since the semantics for $\mathcal{S R O I} 44$ is 4 -valued, distinct notions of subsumption and equivalence naturally arise.

Definition 18. Let $C$ and $D$ be $\mathcal{S R O I Q 4}$ concept descriptions and $K B$ a SROI 4 knowledge base.

1. $C$ is 4-satisfiable wrt $K B$ if there is a 4-model $\mathcal{I}$ of $K B$ such that $p^{+}\left(C^{\mathcal{I}}\right)$ is not empty.

2. $C$ is weakly subsumed by $D$ ( $D$ weakly subsumes $C$ ) wrt $K B$ if $p^{+}\left(C^{\mathcal{I}}\right) \subseteq p^{+}\left(D^{\mathcal{I}}\right)$ in every 4-model $\mathcal{I}$ of $K B$.

3. $C$ is strongly subsumed by $D$ ( $D$ strongly subsumes $C$ ) wrt $K B$ if $p^{+}\left(C^{\mathcal{I}}\right) \subseteq p^{+}\left(D^{\mathcal{I}}\right)$ and $p^{-}\left(D^{\mathcal{I}}\right) \subseteq p^{-}\left(C^{\mathcal{I}}\right)$ in every 4 -model $\mathcal{I}$ of $K B$.

4. $C$ and $D$ are weakly equivalent $(C \equiv D)$ wrt $K B$ iff $p^{+}\left(C^{\mathcal{I}}\right)=p^{+}\left(D^{\mathcal{I}}\right)$ on each 4-model $\mathcal{I}$ of $K B$.

5. $C$ and $D$ are strongly equivalent $(C \leftrightarrow D)$ wrt $K B$ iff $C^{\mathcal{I}}=D^{\mathcal{I}}$ on each 4-model $\mathcal{I}$ of $K B$. 
In Example 13, $\operatorname{Van}$ is 4-satisfiable relative to the knowledge base $K B$ defined there, even though the only models for it assign common elements to both its positive and negative extensions. Similarly, since in every model of $K B$ it holds that $p^{+}\left(\operatorname{Sedan}^{\mathcal{I}}\right) \subseteq p^{+}\left((\neg \text { Van })^{\mathcal{I}}\right)$, regardless of the model $\mathcal{I}$ in question, Sedan is weakly subsumed by $\neg$ Van. It is not strongly subsumed, however. For instance, if the interpretation of Example 14 is modified so that $\operatorname{Sedan}^{\mathcal{I}}=\langle\{c 435\},\{c 435\}\rangle$ but $\operatorname{Van}^{\mathcal{I}}=\left\langle\Delta^{\mathcal{I}},\{c 435\}\right\rangle$, then $K B$ is still satisfied. However the criteria for strong subsumption are not met.

The below sets of claims follow immediately from Definition 18 and the semantics of $\mathcal{S R O I} \mathcal{Q} 4$.

Proposition 19. Let $C$ and $D$ be $\mathcal{S R O I Q} 4$ concept descriptions. The following all hold relative to any $\mathcal{S R O I Q 4}$ knowledge base $K B$.

1. $C$ is 4-unsatisfiable iff $C$ is weakly subsumed by $\perp$.

2. $C$ and $D$ are weakly equivalent iff $C$ weakly subsumes $D$ and $D$ weakly subsumes $C$.

3. $C$ and $D$ are strongly equivalent iff $C$ strongly subsumes $D$ and $D$ strongly subsumes $C$.

Proposition 20. Let $C$ and $D$ be $\mathcal{S R O I Q 4}$ concept descriptions. The following all hold relative to any $\mathcal{S R O I Q 4}$ knowledge base $K B$.

1. $C$ is weakly subsumed by $D$ iff $C \sqsubset D$ is satisfied on each $\mathcal{I}$.

2. $C$ is strongly subsumed by $D$ iff $C \rightarrow D$ is satisfied on each $\mathcal{I}$.

3. $C$ and $D$ are weakly equivalent iff $C \sqsubset D$ and $D \sqsubset C$ are both satisfied on each $\mathcal{I}$.

4. $C$ and $D$ are strongly equivalent iff $C \rightarrow D$ and $D \rightarrow C$ are both satisfied on each $\mathcal{I}$.

\section{1. $\mathcal{S R O I Q} 4$ is Sound}

Every 2-interpretation $\mathcal{I}_{2}$ of a knowledge base $K B$ corresponds to a 4 -interpretation $\mathcal{I}_{4}$, which we will call the 4 -counterpart of $\mathcal{I}_{2}$ :

$-\Delta^{\mathcal{I}_{4}}={ }_{\text {def }} \Delta^{\mathcal{I}_{2}}$.

- For each $a \in N_{I}, a^{\mathcal{I}_{4}}={ }_{\text {def }} a^{\mathcal{I}_{2}}$.

- For each $R \in N_{R}, R^{\mathcal{I}_{4}}={ }_{\text {def }}\left\langle R^{\mathcal{I}_{2}},\left(\Delta^{\mathcal{I}_{2}} \times\right.\right.$ $\left.\left.\Delta^{\mathcal{I}_{2}}\right)-R^{\mathcal{I}_{2}}\right\rangle$.

- For each $C \in N_{C} \cup N_{o}$, or $C=\exists R$.Self, $C^{\mathcal{I}_{4}}={ }_{\text {def }}\left\langle C^{\mathcal{I}_{2}}, \Delta^{\mathcal{I}_{2}}-C^{\mathcal{I}_{2}}\right\rangle$.

Since $\Delta^{\mathcal{I}_{4}}={ }_{\text {def }} \Delta^{\mathcal{I}_{2}}$, we will use $\Delta$ to refer to the common domain of discourse. In the counterpart $\mathcal{I}_{4}$, the positive and negative extensions of each atomic concept partition $\Delta$. In fact, this holds for even non-atomic concept descriptions.

Proposition 21. If $\mathcal{I}_{2}$ is a 2-interpretation and $\mathcal{I}_{4}$ its 4-counterpart, then for any concept description $C, C^{\mathcal{I}_{4}}=\left\langle C^{\mathcal{I}_{2}}, \Delta-C^{\mathcal{I}_{2}}\right\rangle$.

Proposition 22. If $\mathcal{I}_{2}$ is a 2-interpretation and $\mathcal{I}_{4}$ its 4-counterpart, then for any axiom $A, \mathcal{I}_{4}$ is a 4-model of $A$ iff $\mathcal{I}_{2}$ is a 2-model of $A$.

Given Proposition 22 and the fact that concept inclusion axioms have the same semantics in $\mathcal{S R O} \mathcal{I} \mathcal{Q}$ regardless of their type, the following are all equivalent:

- $\mathcal{I}_{2}$ is a 2-model of $C \sqsubseteq D$.

- $\mathcal{I}_{4}$ is a 4 -model of $C \sqsubset D$.

- $\mathcal{I}_{4}$ is a 4-model of $C \mapsto D$.

- $\mathcal{I}_{4}$ is a 4 -model of $C \rightarrow D$.

Example 23. Let $K B_{2}$ be the knowledge base from Example 13 with the assertion Van(c435) removed. $K \mathrm{~B}_{2}$ is classically satisfiable, and we may define a 2-valued model $\mathcal{I}_{2}$ as follows:

$-\Delta=\{c 435, i 90$, tom, unit 1$\}$

$-c 435^{\mathcal{I}_{2}}=c 435, i 90^{\mathcal{I}}=i 90$,

tom $^{\mathcal{I}_{2}}=$ tom, unit $^{\mathcal{I}}=$ unit 1

- Sedan ${ }^{\mathcal{I}_{2}}=\{c 435\}$

$-\operatorname{Van}^{\mathcal{I}_{2}}=\varnothing$

- Incident $^{\mathcal{I}}=\{i 90\}$

- involvedIn ${ }^{\mathcal{I}}=\{(c 435, i 90)\}$

- assignedTo $o^{\mathcal{I}}=\{($ unit $1, i 90)\}$

- memberOf $f^{\mathcal{I}}=\{($ tom, unit 1$)\}$

The 4-valued counterpart $\mathcal{I}_{4}$ of $\mathcal{I}_{2}$ looks exactly like the 4 -valued interpretation in Example 14 save that interpretations for $\operatorname{Van}$ and Sedan are as shown below.

$$
\begin{aligned}
& -\operatorname{Sedan}^{\mathcal{I}}=\left\langle\{c 435\}, \Delta^{\mathcal{I}}-\{c 435\}\right\rangle \\
& -\operatorname{Van}^{\mathcal{I}}=\left\langle\varnothing, \Delta^{\mathcal{I}}\right\rangle
\end{aligned}
$$

Given this, $(\neg \operatorname{Van})^{\mathcal{I}_{4}}$ is $\left\langle\Delta^{\mathcal{I}}, \varnothing\right\rangle$, which is in agreement with Proposition 21. Furthermore, it can be readily verified that $\mathcal{I}_{4}$ is a 4 -model of $K B_{2}$, in agreement with Proposition 22

Proposition 24. Let $K B$ be a $\mathcal{S R O I Q 4}$ knowledge base and $A$ a SROIQ4 axiom. If $K B=\mathcal{S R O I Q 4}$ $A$, then $K B=\mathcal{S R O I} \mathcal{Q} A$.

Proof. If $K B={ }_{\mathcal{S R O} \mathcal{I} Q 4} A$ and $\mathcal{I}_{2}$ 2-satisfies $K B$, then by Prop. 22 the 4-counterpart $\mathcal{I}_{4}$ of $\mathcal{I}_{2} 4$ satisfies $K B$ and hence $A$. Again by Prop. 22, $\mathcal{I}_{2}$ 2-satisfies $A$. 
And so $\mathcal{S R O I Q 4}$ is sound wrt $\mathcal{S R O I Q}$.

Example 25. In Examples 13 and 23, one may conclude $\neg$ Van $(v 435)$ (which follows trivially by Modus Ponens). In Example 13, one may also conclude Van(435) (by Reflexivity). In both cases, these conclusions are sound relative to classical $\mathcal{S R O I Q}$ (in Example 13 , since $K B$ is classically inconsistent, everything follows from it).

Significantly, regardless of what inclusion operator is used in $\mathcal{S R O I Q 4}$, if $A$ is entailed in $\mathcal{S R O I Q 4}$, then it is also entailed according to $\mathcal{S R O I} \mathcal{Q}$. We can show this by replacing each inclusion symbol with $\sqsubseteq$.

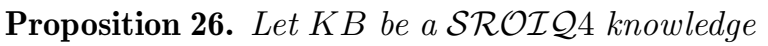
base, $A$ a $\mathcal{S R O I Q 4}$ axiom, and $K B^{\prime}$ and $A^{\prime}$ the

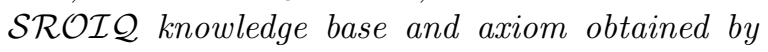
replacing each occurrence of $\sqsubset, \mapsto$, and $\rightarrow$ with $\sqsubseteq$.

$$
\text { If } K B=\operatorname{SROI}_{\mathcal{Q} 4} A \text {, then } K B^{\prime}=\operatorname{SROI} \mathcal{Q}^{\prime}
$$

Proof. If $K B={ }_{\mathcal{S R O} \mathcal{I}_{\mathcal{Q}} 4} A$ and $\mathcal{I}_{2} 2$-satisfies $K B^{\prime}$, then by Prop. 22, the 4-counterpart $\mathcal{I}_{4}$ of $\mathcal{I}_{2}$ 4satisfies $K B^{\prime}$. Furthermore (by Prop. 22), $\mathcal{I}_{4} 4$ satisfies $K B$. Since $K B \models \mathcal{S R O}_{\mathcal{O} \mathcal{I}_{\mathcal{Q}} A} A, \mathcal{I}_{4} 4$-satisfies $A$, and so by Prop. 22, $\mathcal{I}_{2} 2$-satisfies $A$. If $A \neq$ $A^{\prime}$, then $A$ must be a $\mathcal{S} \mathcal{R O} \mathcal{I} \mathcal{Q} 4$ inclusion axiom, which by convention has the same semantics in $\mathcal{S} \mathcal{R O} \mathcal{I}$ as $A^{\prime}$. And so $\mathcal{I}_{2} 2$-satisfies $A^{\prime}$.

Example 27. Consider the set $K B$

$$
\{\operatorname{Sedan}(c 435), \operatorname{Van}(c 435), \text { Sedan } \sqsubseteq \neg \operatorname{Van}\}
$$

of axioms from Example 13. One can conclude $\neg \operatorname{Van}(c 435)$ but cannot conclude $\neg \operatorname{Sedan}(c 435)$, as internal inclusion does not permit Modus Tollens. However, the latter expression does follow if Sedan $\sqsubset \neg V$ an is replaced with Sedan $\rightarrow \neg V$ an . In both cases, the conclusions are sound relative

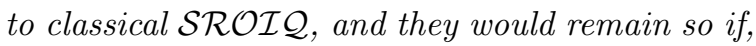
e.g., Sedan(c435) were removed in the second case to make the knowledge base consistent.

Recall the two accounts of equivalence given earlier. Strong equivalence corresponds most closely to the classical account, and it is the account used in Arieli and Avron's logic. It is often more than one needs, however, since satisfaction and entailment in $\mathcal{S R O I Q} 4$ are defined using only the positive extensions of axioms. E.g.,

$$
\begin{aligned}
& \{C(a)\}={ }_{S \mathcal{R O} \mathcal{I Q}_{4}} D(a) \text { and } \\
& \{D(a)\} \models \mathcal{S R O I} C(a)
\end{aligned}
$$

holds iff $C$ and $D$ are weakly equivalent. Furthermore, because $\mathcal{S R O I} 4$ is sound wrt $\mathcal{S R O I Q}$, if

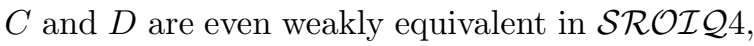
then the concepts are equivalent in classical $\mathcal{S R O} \mathcal{I} \mathcal{Q}$. Something similar holds for disjointness. For instance, if

$$
\begin{aligned}
& K B=\operatorname{SROI}_{\mathcal{Q} 4} C \sqsubset \neg D \text { or } \\
& K B=\mathcal{S R O} \mathcal{I}_{\mathcal{Q} 4} D \sqsubset \neg C,
\end{aligned}
$$

then $C$ and $D$ are classically disjoint wrt $K B$.

\section{Removing Gaps and Gluts}

A paraconsistent logic typically rejects one or both of the below traditional laws.

- Law of Noncontradiction (LNC): $\neg(\mathcal{P} \wedge \neg \mathcal{P}$ )

- Law of the Excluded Middle (LEM): $\mathcal{P} \vee \neg \mathcal{P}$

$\mathcal{S R O I} 4$ rejects both.

Example 28. Consider $K B=\{\top(a)\}$. In a classical setting, one could infer that $a$ is a member of Person $\sqcup \neg$ Person. However, in the 4valued setting, this is not the case. E.g., $a^{\mathcal{I}} \notin$ $p^{+}\left((\text {Person } \sqcup \neg \text { Person })^{\mathcal{I}}\right)$ in any 4-valued interpretation $\mathcal{I}$ in which Person $\mathcal{I}^{\mathcal{I}}=\langle\varnothing, \varnothing\rangle$. Similarly, in the classical setting, one would be able to infer that a is not a member of Person $\sqcap \neg$ Person. However, (Person $\sqcap \neg$ Person)(a) is satisfied in any 4-valued interpretation $\mathcal{I}$ in which Person $^{\mathcal{I}}=$ $\langle\Delta, \Delta\rangle$.

Nevertheless, inserting additional axioms into a $\mathcal{S R O I} 4$ knowledge base can selectively enforce the two laws, effectively making the knowledge base "more classical." In particular, if LEM is enforced but not LNC, then additional classical consequences can be drawn from the knowledge base while at the same time maintaining paraconsistency.

Following Arieli [3], we define the sets $E M(K B)$ ("excluded middle") and $E F Q(K B)$ ("ex falso quodlibet"). In any 4-model of $K B \cup E M(K B)$, LEM holds for concept assertions: For any individual $a$ and atomic concept $A$, either $A(a)$ or $\neg A(a)$ is satisfied. Similarly, in any 4-model of $K B \cup E F Q(K B)$, LNC holds: At most one of $A(a)$ or $\neg A(a)$ is satisfied.

Definition 29. Let $K B$ be a $\mathcal{S R O I Q} 4$ knowledge base: 

- $E M(K B)=_{\operatorname{def}}\{\top \sqsubset(A \sqcup \neg A): A=$ $\exists$ R.Self or $\left.A \in N_{C} \cup N_{o}\right\}$.
$-E F Q(K B)=_{\text {def }}\{(A \sqcap \neg A) \sqsubset \perp: A=$ $\exists$ R.Self or $\left.A \in N_{C} \cup N_{o}\right\}$.

Proposition 30. $\mathcal{I}$ is a 4-model of $E M(K B)$ iff for each concept $C$ of $K B$,

$$
p^{+}\left(C^{\mathcal{I}}\right) \cup p^{-}\left(C^{\mathcal{I}}\right)=\Delta^{\mathcal{I}} .
$$

Proposition 31. $\mathcal{I}$ is a 4-model of $E F Q(K B)$ iff for each concept $C$ of $K B$,

$$
p^{+}\left(C^{\mathcal{I}}\right) \cap p^{-}\left(C^{\mathcal{I}}\right)=\varnothing .
$$

In 3], adding the analog of $\operatorname{EM}(K B)$ to Arieli's logic yields Kleene's $K_{3}$, while adding the analog of $E F Q(K B)$ yields $L P$. The maneuver here can be seen as simulating similar logics in the DL setting. Both of these logics are deductively stronger than $\mathcal{S R O I Q 4}$. Since the purpose of $\mathcal{S R O I Q 4}$ is to reason paraconsistently, adding $\operatorname{EM}(K B)$ to a knowledge base brings $\mathcal{S R O I} Q 4$ closer to $\mathcal{S R O I Q}$ without abandoning that objective.

Adding both $E M(K B)$ and $E F Q(K B)$ allow

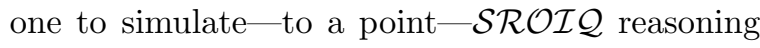
in $\mathcal{S R O I}$ Q4. Let $\mathcal{I}_{4}$ be a 4-interpretation of $K B$. We define a corresponding 2-interpretation $\mathcal{I}_{2}$ as shown below. Only the positive extensions are used in the construction.

$-\Delta^{\mathcal{I}_{2}}=$ def $\Delta^{\mathcal{I}_{4}}$.

- For each individual $a \in N_{I}, a^{\mathcal{I}_{2}}=\operatorname{def} a^{\mathcal{I}_{4}}$.

- For each role $R \in N_{R}, R^{\mathcal{I}_{2}}=$ def $p^{+}\left(R^{\mathcal{I}_{4}}\right)$.

- For each $C \in N_{C} \cup N_{o}, C^{\mathcal{I}_{2}}=\operatorname{def} p^{+}\left(C^{\mathcal{I}_{4}}\right)$.

Proposition 32. $\mathcal{I}_{4}$ is a 4-model of $\operatorname{EM}(K B) \cup$ $E F Q(K B)$ iff for each concept $C$,

$$
C^{\mathcal{I}_{4}}=\left\langle C^{\mathcal{I}_{2}}, \Delta-C^{\mathcal{I}_{2}}\right\rangle .
$$

Proposition 33. Let $\mathcal{I}_{4}$ be a 4-model of $E M(K B) \cup$ $E F Q(K B)$. If $A$ is a $\mathcal{S R O I} \mathcal{Q} 4$ axiom of $K B$ and not of the form $\neg R(a, b), \operatorname{Irr}(R)$, or $\operatorname{Dis}(R, S)$, then $\mathcal{I}_{2}$ is a 2-model of $A$ iff $\mathcal{I}_{4}$ is a 4-model of $A$.

Proposition 34. If $K B$ is a $\mathcal{S R O I Q 4 ~ k n o w l e d g e ~}$ base lacking axioms of the form $\neg R(a, b), \operatorname{Irr}(R)$, or $\operatorname{Dis}(R, S)$, then

$$
K B \cup E M(K B) \cup E F Q(K B)
$$

has a 4-model iff KB has a 2-model.
Example 35. Consider the set $K B$

$$
\{\operatorname{Sedan}(c 435), \operatorname{Van}(c 435), \text { Sedan } \sqsubseteq \neg \operatorname{Van}\}
$$

of axioms from Example 13 , and let $\mathcal{I}_{4}$ be defined as follows: $\Delta^{\mathcal{I}_{4}}=\{c 435\}, c 435^{\mathcal{I}_{4}}=c 435$, and Sedan ${ }^{\mathcal{I}_{4}}=\operatorname{Van}^{\mathcal{I}_{4}}=\langle\{c 435\},\{c 435\}\rangle . \mathcal{I} 4$ is a 4 valued model the axioms. However, it is not a 4valued model of (Van $\sqcap \neg$ Van $) \sqsubseteq \perp$, and so it is not a 4-valued model of $E F Q(K B)$.

The 2-valued counterpart $\mathcal{I}_{2}$ of $\mathcal{I}_{4}$ is: $\Delta^{\mathcal{I}_{2}}=$ $\{c 435\}, c 435^{\mathcal{I}_{2}}=c 435$, Sedan ${ }^{\mathcal{I}_{2}}=\operatorname{Van}^{\mathcal{I}_{2}}=$ $\{c 435\}$. This is not a 2-valued model of $K B$ (the knowledge base has no such models). As implied by Proposition 34, $K B \cup E F Q(K B)$ has no 4valued models. This can be seen by noting that $c 435^{\mathcal{I}} \in p^{+}\left(\operatorname{Van}^{\mathcal{I}}\right) \cap p^{-}\left(\operatorname{Van}^{\mathcal{I}}\right)$ in any 4-valued model $\mathcal{I}$ of $K B$.

Propositions 33 and 34 do not allow the role assertions above because we cannot add axioms for roles similar to $E M(K B)$ and $E F Q(K B)$. Such constructions involving roles are not allowed in $\mathcal{S R O I} \mathcal{Q}$ or $\mathcal{S R O I} Q 4$. If roles are taken as bivalent, then obviously the ban can be lifted ${ }^{2}$

\section{Embedding $\mathcal{S R O I} Q 4$ into $S \mathcal{R O I} \mathcal{Q}$}

To allow the use of classical DL reasoners with $\mathcal{S R O I}$ Q4, we provide a translation function $\pi$

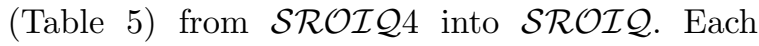
atomic concept $C$ in a $\mathcal{S R O I} 4$ knowledge base is left untouched, but the negated concept $\neg C$ becomes the new atomic concept $C^{\prime}$. In this way the 4-satisfiable concept $C \sqcap \neg C$ becomes the 2satisfiable $C \sqcap C^{\prime} 3^{3}$ Below, $\mathcal{L}$ is used to refer to the

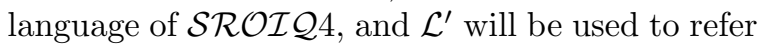
to the language created by adding primed counterparts to all atomic roles and concepts in $\mathcal{L}$. We also add new atomic concepts $C_{R . S e l f}$ for each $R$ in $\mathcal{L}$, and $C_{o}$ for each nominal $o \in N_{o}$. These will be used to stand for the negations of R.Self and nominals, respectively.

It is clear that if $K B$ is a $\mathcal{S R O I Q 4}$ knowledge base, then $\pi(K B)$ is a $\mathcal{S R O I} \mathcal{Q}$ knowledge

\footnotetext{
${ }^{2}$ Alternatively, one could attempt to address this issue by enhancing the language with further constructs for roles, as is done in 65. However, the details of this remain to be spelled out.

${ }^{3}$ The basic approach is based on a method of translating paraconsistent propositional logics into classical logic [7/3.
} 
base. Furthermore, since the transformation does not syntactically affect role inclusion axioms, if the role hierarchy is regular in $K B$, then it will be regular in $\pi(K B)$. Similarly, the counterpart of a simple role in $K B$ is itself simple in $\pi(K B)$. As this is so, it is assured that $\pi$ does not lead to undecidability.

As equality between individuals is interpreted classically in $\mathcal{S R O I} \mathcal{P} 4$, the most obvious translation of cardinality restrictions sometimes leads to unnecessary inconsistencies. Example 36 below illustrates how $\pi$ avoids it. Section 6 shows that nominals cause a similar difficulty. In that case, however, the inconsistencies cannot be removed.

Example 36 (46], Ex. 1). The assertion

$$
((\geq 2 R . C) \wedge(\leq 1 R . C))(a)
$$

is 4-satisfiable but not 2-satisfiable. If the following scheme is used to translate cardinality restrictions

$$
\begin{aligned}
& \pi(\leq n R . C)=\leq n R . \pi(C) \\
& \pi(\neg \leq n R . C)=\geq(n+1) R . \pi(C) \\
& \pi(\geq n R . C)=\geq n R . \pi(C) \\
& \pi(\neg \geq n R . C)=\leq(n-1) R . \pi(C)
\end{aligned}
$$

then the assertion remains unchanged and so classically unsatisfiable. The actual translation $\pi$, however, yields the 2-satisfiable $(\geq 2 R . C) \wedge(\leq$ $\left.1 R . \neg C^{\prime}\right)$. While concepts $\neg C^{\prime}$ and $C$ intuitively refer to the same thing, they are formally distinct.

In general, the relationship between $K \models \mathcal{P}$ and the unsatisfiability of $K \cup\{\neg \mathcal{P}\}$ does not hold in the paraconsistent context, and so entailment cannot be reduced to satisfiability checking. Nevertheless, there is a strong and useful relationship between $K B$ and $\pi(K B)$ that allows the use of satisfiability. If $K B$ entails $A$ in $\mathcal{S R O I Q 4 , ~ t h e n ~}$ $\pi(K B)$ entails $\pi(A)$ in $\mathcal{S R O \mathcal { I }}$ (Proposition 39). From this, it follows that $\pi(K B) \cup\{\neg \pi(A)\}$ is classically inconsistent.

Let $\mathcal{L}$ be the language of $\mathcal{S R O I Q 4}$. If $\mathcal{I}$ is a 4 -interpretation of $\mathcal{L}$, then define the 2 interpretation $\mathcal{I}^{\prime}$ (the primed counterpart of $\mathcal{I}$ ) over $\mathcal{L}^{\prime}$ as follows:

$-\Delta^{\mathcal{I}^{\prime}}={ }_{\text {def }} \Delta^{\mathcal{I}}$.

- For each individual $a \in N_{I}, a^{\mathcal{I}^{\prime}}={ }_{\text {def }} a^{\mathcal{I}}$.

- For each role $R \in N_{R} \cup N_{R}^{-}, R^{\mathcal{I}^{\prime}}={ }_{\text {def }} p^{+}\left(R^{\mathcal{I}}\right)$ and $R^{\prime \mathcal{I}^{\prime}}={ }_{\text {def }} p^{-}\left(R^{\mathcal{I}}\right)$.

- For each atomic concept $C \in N_{C}, C^{\mathcal{I}^{\prime}}={ }_{\text {def }}$ $p^{+}\left(C^{\mathcal{I}}\right)$ and $C^{\prime \mathcal{I}^{\prime}}={ }_{\text {def }} p^{-}\left(C^{\mathcal{I}}\right)$.
- For each nominal $o \in N_{O}, o^{\mathcal{I}^{\prime}}=\operatorname{def} p^{+}\left(C^{\mathcal{I}}\right)$, and $C_{o}^{\mathcal{I}^{\prime}}={ }_{\text {def }} p^{-}\left(o^{\mathcal{I}}\right)$.

- For each role $R \in N_{R} \cup N_{R}^{-},(\exists R \text {.Self })^{\mathcal{I}^{\prime}}={ }_{\text {def }}$ $p^{+}\left((\exists R . S e l f)^{\mathcal{I}}\right)$, and

- For each role $R \in N_{R} \cup N_{R}^{-}, C_{R . S e l f}^{\mathcal{I}^{\prime}}={ }_{\text {def }}$ $p^{-}\left((\exists R . S e l f)^{\mathcal{I}}\right)$.

It is clear that there is a $1-1$ correspondence between the 4 -interpretations of $\mathcal{L}$ and the 2interpretations of $\mathcal{L}^{\prime}$.

Proposition 37. For any 4-interpretation $\mathcal{I}$ with

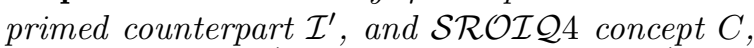
$p^{+}\left(C^{\mathcal{I}}\right)=\pi(C)^{\mathcal{I}^{\prime}}$ and $p^{-}\left(C^{\mathcal{I}}\right)=\pi(\neg C)^{\mathcal{I}^{\prime}}$ both hold.

Proposition 38. For any 4-interpretation $\mathcal{I}, \mathcal{I}$ is a 4-model of $\mathcal{S} \mathcal{R O I} 44$ axiom $A$ iff its primed counterpart $\mathcal{I}^{\prime}$ is a 2-model of $\pi(A)$.

Proposition 39. For any $\mathcal{S} \mathcal{R O I} 44$ knowledge base $K B$ and axiom $A, K B \models \mathcal{S R O I} 4 \quad A$ iff $\pi(K B) \models \mathcal{S R O}_{\mathcal{I} \mathcal{Q}} \pi(A)$.

It is Proposition 39 that shows that reasoning with $\mathcal{S} \mathcal{R O} \mathcal{I} Q 4$ can be performed using the translation $\pi$ and a classical reasoner.

Example 40. Consider the set $\{\operatorname{Sedan}(c 435)$, Van(c435), Sedan $\sqsubset \neg$ Van, Sedan $\sqsubseteq$ Vehicle $\}$. In any 4-valued model $\mathcal{I}$, it must be the case that $c 435^{\mathcal{I}} \in p^{+}\left(\operatorname{Sedan}^{\mathcal{I}}\right)$ and $c 435^{\mathcal{I}} \in p^{+}\left(\operatorname{Van}^{\mathcal{I}}\right)$, and also $c 435^{\mathcal{I}} \in p^{+}\left((\neg V \text { an })^{\mathcal{I}}\right)$ and $c 435^{\mathcal{I}} \in$ $p^{+}\left(\right.$Vehicle $\left.^{\mathcal{I}}\right)$. As such, $c 435^{\mathcal{I}^{\prime}} \in$ Sedan $^{\mathcal{I}^{\prime}}, c 435^{\mathcal{I}^{\prime}}$ $\in \operatorname{Van}^{\mathcal{I}^{\prime}}, c 435^{\mathcal{I}^{\prime}} \in \operatorname{Sedan}^{\mathcal{I}^{\prime}}$, and $c 435^{\mathcal{I}^{\prime}} \in \operatorname{Van}^{\mathcal{I}^{\prime}}$, where $\mathcal{I}^{\prime}$ is the primed-counterpart of $\mathcal{I}$ and $V$ an' is $\pi(\neg$ Van $)$. Note that $\pi($ Sedan $\sqsubset \neg$ Van $)=$ Sedan $\sqsubseteq V a n^{\prime}$, and so it is clear that $\mathcal{I}^{\prime}$ is a 2valued model of $\pi$ (Sedan $\sqsubset \neg$ Van). It is trivial to determine that $\mathcal{I}^{\prime}$ must be a 2-valued model of $\pi(K B)$ (Proposition 38).

In agreement with Proposition $37, p^{+}\left(\operatorname{Van}^{\mathcal{I}}\right)=$ $\operatorname{Van}^{\mathcal{I}^{\prime}}=\pi(\text { Van })^{\mathcal{I}^{\prime}}$ and $p^{-}\left(\right.$Van $\left.^{\mathcal{I}}\right)=\pi(\neg \text { Van })^{\mathcal{I}^{\prime}}$. Also, Vehicle (c435) is entailed both by the original knowledge base under the 4-valued semantics and by the translated knowledge base under the 2valued semantics, in accordance with Proposition 39

\section{Partial Paraconsistency}

$\mathcal{S R O I} 44$ is intended to avoid the explosions caused by inconsistencies. Unfortunately, some 
Table 5

Translation of $\mathcal{S R O I Q} 4$ into $\mathcal{S R O} \mathcal{I} \mathcal{Q}$.

\begin{tabular}{|c|c|}
\hline $\begin{array}{l}\pi(C)=C, \text { where } C \in N_{C} \\
\pi(o)=o, \text { where } o \in N_{o} \\
\pi(\top)=\top \\
\pi(\perp)=\perp\end{array}$ & $\begin{array}{l}\pi(\neg C)=C^{\prime}, \text { where } C^{\prime} \text { new } \\
\pi(\neg o)=C_{o}, o \in N_{o} \text { and } C_{o} \text { new } \\
\pi(\neg \neg C)=\pi(C) \\
\pi(\neg \top)=\perp \\
\pi(\neg \perp)=\top\end{array}$ \\
\hline $\begin{array}{l}\pi(E \sqcap D)=\pi(E) \sqcap \pi(D) \\
\pi(E \sqcup D)=\pi(E) \sqcup \pi(D)\end{array}$ & $\begin{array}{l}\pi(\neg(E \sqcap D))=\pi(\neg E) \sqcup \pi(\neg D) \\
\pi(\neg(E \sqcup D))=\pi(\neg E) \sqcap \pi(\neg D)\end{array}$ \\
\hline $\begin{array}{l}\pi(\forall R . C)=\forall R . \pi(C) \\
\pi(\exists R . C)=\exists R . \pi(C) \\
\pi(\exists R . \text { Self })=\exists \text { R.Self }\end{array}$ & $\begin{array}{l}\pi(\neg(\forall R . C))=\exists R . \pi(\neg C) \\
\pi(\neg(\exists R . C))=\forall R . \pi(\neg C) \\
\pi(\neg \exists R . \text { Self })=C_{R . S e l f}\end{array}$ \\
\hline $\begin{array}{l}\pi(\leq n R . C)=\leq n R . \neg \pi(\neg C) \\
\pi(\geq n R . C)=\geq n R . \pi(C)\end{array}$ & $\begin{array}{l}\pi(\neg \leq n R . C)=\geq(n+1) R . \pi(C) \\
\pi(\neg \geq n R . C)=\leq(n-1) R . \neg \pi(\neg C)\end{array}$ \\
\hline $\begin{array}{l}\pi(C(a))=\pi(C)(a) \\
\pi(R(a, b))=R(a, b) \\
\pi(\operatorname{Ref}(R))=\operatorname{Ref}(R) \\
\pi(\operatorname{Dis}(R, S))=\left\{R \sqsubseteq S^{\prime}, S \sqsubseteq R^{\prime}\right\}\end{array}$ & $\begin{array}{l}\pi(a \neq b)=(a \neq b) \\
\pi(\neg R(a, b))=R^{\prime}(a, b) \\
\pi(\operatorname{Irr}(R))=\operatorname{Ref}\left(R^{\prime}\right)\end{array}$ \\
\hline $\begin{array}{l}\pi(C \mapsto D)=\neg \pi(\neg C) \sqsubseteq \pi(D) \\
\pi(C \sqsubset D)=\pi(C) \sqsubseteq \pi(D) \\
\pi(C \rightarrow D)=\{\pi(C \sqsubset D), \pi(\neg D \sqsubset \neg C)\} \\
\pi\left(w \sqsubseteq R_{n+1}\right)=w \sqsubseteq R_{n+1}, \text { with } w=R_{1} \circ \ldots \circ R_{n}\end{array}$ & $\begin{array}{l}\text { material inclusion } \\
\text { internal inclusion } \\
\text { strong inclusion } \\
\text { role inclusion }\end{array}$ \\
\hline
\end{tabular}

$\mathcal{S R O I Q} 4$ knowledge bases-e.g., $\{(\top \sqcap \perp)(a)\}$ have no 4-models, and so $\mathcal{S R O} \mathcal{I} \mathcal{Q} 4$ is only "partially" paraconsistent. In some cases, inconsistent knowledge bases can be re-written into a classically equivalent form that avoids explosion. However, this is not the case in general.

In 50, it was reported that consistency can be maintained in $\mathcal{S H I} \mathcal{I} 4$ by replacing $T$ and $\perp$ with classically equivalent formulas. Specifically, let $S F(K B)$ be the knowledge base obtained by replacing each $\top$ with $A \sqcup \neg A$ and each $\perp$ with $A \sqcap \neg A$ (where $A$ is a new atomic concept). $S F(K B)$ is guaranteed to possess a 4 -model. However, nominals can conflict with cardinality restrictions, and so the analogous claim does not hold for $\mathcal{S R O I} 4$ 4. For instance, any assertion of the form $\geq n+1 R .\left\{a_{1}, \ldots, a_{n}\right\}(b)$ has no 4 -valued models $4^{4}$ More simply, nominals can directly conflict with inequality assertions.

Example 41. Suppose that the unique name assumption is enforced in a given knowledge base for days of the week (using axioms monday $\neq$

\footnotetext{
${ }^{4}$ This is so regardless of whether nominals are interpreted classically or as done here.
}

tuesday, tuesday $\neq$ wednesday, etc.). Then the following set of assertions has no 4-valued models.

AvailableDay $\subseteq\{$ monday, wednesday $\}$, AvailableDay(tuesday)

In order to maintain satisfiability, either cardinality restrictions (and inequality assertions with them) or nominals must go.

Proposition 42. If $C$ is a concept description in which nominals, $\top$, and $\perp$ do not appear, then $C$ is 4-satisfiable.

Proof. Let $n$ be the largest integer used in an $\geq$ $n R . C$ restriction. Let $C^{\mathcal{I}}=\langle\Delta, \Delta\rangle$ for each $C \in$ $N_{C}$, where $|\Delta|=n+1$. For each $R \in N_{R}$, let $R^{\mathcal{I}}=$ $\left\langle\Delta^{2}, \Delta^{2}\right\rangle$ and $(\exists R \text {.Self })^{\mathcal{I}}=\langle\Delta, \Delta\rangle$. We induct on a the degree of $C$. If $C \in N_{C}$, the claim clearly holds. Examining the $\mathcal{S R O} \mathcal{I} \mathcal{Q} 4$ semantics shows that where $C$ is $\neg D, D \sqcup E, D \sqcap E, \forall R . D, \exists R . D$, $\geq n R . D$, or $\leq n R . D, C^{\mathcal{I}}=\langle\Delta, \Delta\rangle$.

Proposition 43. If $K B$ is a $\mathcal{S R O I Q 4 ~ k n o w l e d g e ~}$ base in which inequality assertions and nominals do not appear, then $S F(K B)$ has a 4-model.

Proof. We use the interpretation $\mathcal{I}$ above. For simple assertions $C(a)$ and $R(a, b)$, the claim obvi- 
ously holds. Since the anti-extension of each role $R$ is $\Delta^{2}, \neg R(a, b)$ also holds (we here reasonably assume that $R \neq U)$, as do $\operatorname{Ref}(R), \operatorname{Irr}(R)$, and $\operatorname{Dis}(R, S)$. Since for all concepts $C$ and $D, C^{\mathcal{I}}=$ $D^{\mathcal{I}}=\langle\Delta, \Delta\rangle$, it follows that $C \sqsubset D$ and $C \rightarrow D$ hold. For $C \mapsto D$, observe that $\Delta^{\mathcal{I}}-p^{-}\left(C^{\mathcal{I}}\right)=\varnothing$, and so $C \mapsto D$ must be true in $\mathcal{I}$. The cases for RIAs are similar to $C \sqsubset D$.

Proposition 44. If $C$ is a $\mathcal{S R O I Q 4}$ concept description in which $\leq, \geq, \top$, and $\perp$ do not appear, then $C$ is 4-satisfiable.

Proof. Let $\Delta^{\mathcal{I}}=\{d\}, C^{\mathcal{I}}=\langle\{d\},\{d\}\rangle$ for each $C \in N_{C} \cup N_{o}$, and $a^{\mathcal{I}}=d$ for each $a \in N_{I}$. For each $R \in N_{R}$, let $R^{\mathcal{I}}=\langle\{(d, d)\},\{(d, d)\}\rangle$ and $(\exists R . S e l f)^{\mathcal{I}}=\langle\{d\},\{d\}\rangle$. Inducting on the degree of $C$, if $C \in N_{C} \cup N_{o}$ or $C=\exists R$.Self, then the claim holds. Examining the semantics of the concept description operators shows that the claim holds for $\neg C, C \sqcup D, C \sqcap D, \exists R . C$, and $\forall R . C$.

Proposition 45. If $K B$ is a $\mathcal{S R O I Q 4}$ knowledge base in which inequality assertions, $\leq$, and $\geq$, do not appear, then $S F(K B)$ has a 4-model.

Proof. Using $\mathcal{I}$ above, consider each type of axiom. For $C(a)$ and $R(a, b)$, the claim obviously holds. Since $p^{-}\left(R^{\mathcal{I}}\right)=\{(d, d)\}, \neg R(a, b)$ also holds (we assume that $R \neq U)$. It is clear that $\operatorname{Re} f(R)$, $\operatorname{Irr}(R)$, and $\operatorname{Dis}(R, S)$ also hold. Since for all concepts $C$ and $D, C^{\mathcal{I}}=D^{\mathcal{I}}=\langle\{d\},\{d\}\rangle, C \sqsubset D$ and $C \rightarrow D$ hold. For $C \mapsto D, \Delta^{\mathcal{I}}-p^{-}\left(C^{\mathcal{I}}\right)=\varnothing$, and so $\mathcal{I} 4$-satisfies $C \mapsto D$. The cases for RIAs proceed similarly.

One obvious solution to the conflict between cardinality restrictions and inequality assertions, on the one hand, and nominals on the other is to use pseudo-nominals in place of real nominals. E.g., instead of specifying the days of the week with the nominal $\{$ monday, tuesday,...\}, we represent the individuals with classes Monday $\sqcup$ Tuesday $\sqcup$.... In practice, this is sometimes done. However, it is well known that the ontology obtained via the substitution is not classically equivalent to the original, and furthermore that the use of pseudo-nominals leads to intuitively incorrect results. It's the same in the 4-valued context. However, to achieve paraconsistency, something of the classical logic must be abandoned, and for many applications, the loss of real nominals might be acceptable.

\section{Tractable DLs}

The latest revision of the Web Ontology Language (OWL 2) 30] features profiles having polynomial time complexities [57]. Below, we examine the tractable languages upon which these profiles are based. In particular, we examine $\mathcal{E} \mathcal{L}^{++}$, which corresponds to OWL 2 EL; DL-Lite, which

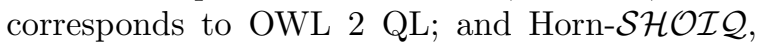
which is an extension of OWL 2 RL. We focus on whether reasoning with the four-valued semantics presented earlier can preserve the tractability of these logics. Specifically, we focus on whether the reduction to classical logics yields formulas still within a tractable logic. It turns out that it does, provided internal inclusion is used. For material and strong inclusion, however, the reduction produces formulas outside of the tractable fragment.

\section{1. $\mathcal{E} \mathcal{L}^{++}$}

The syntax of $\mathcal{E} \mathcal{L}^{++}$, which restricts that of $\mathcal{S R O I} \mathcal{Q}$, is shown in Table 6. RIAs of the form

$$
R \sqsubseteq S \text { and } R_{1} \circ R_{2} \circ \ldots \circ R_{n} \sqsubseteq S,
$$

where $R_{i}, S \in N_{R}$, are also allowed. Expressions involving concrete domains are permitted, too, but we do not consider them here. The semantics of $\mathcal{E} \mathcal{L}^{++}$is the same as that of $\mathcal{S R O \mathcal { Q }}$.

The knowledge base $\{A \sqsubseteq \perp, A(a)\}$ shows that $\mathcal{E} \mathcal{L}^{++}$knowledge bases can be inconsistent. Inconsistency is caused specifically by the presence of $\perp$, and consistency can be restored by replacing each occurrence of $\perp$ with the classically equivalent $A_{\perp} \sqcap \neg A_{\perp}$ (where $A_{\perp}$ is a new atomic concept). Doing this is unproblematic: since the negation appears in front of an atomic concept, it will be eliminated when processed by $\pi$. In general, applying $\pi$ to an $\mathcal{E} \mathcal{L}^{++}$concept (or a concept containing $\neg A$ ) produces an $\mathcal{E} \mathcal{L}^{++}$concept.

Unfortunately, the use of the paraconsistent inclusion operators causes problems. Applying $\pi$ to $C \sqsubset D$ yields $\pi(C) \sqsubseteq \pi(D)$, where $\pi(C)$ and $\pi(D)$ are $\mathcal{E} \mathcal{L}^{++}$concepts. As such, $\pi(C) \sqsubseteq \pi(D)$ is still an $\mathcal{E} \mathcal{L}^{++}$axiom, and so internal inclusion does not destroy the tractability of $\mathcal{E} \mathcal{L}^{++}$. However, applying $\pi$ to GCIs using $\rightarrow$ and $\mapsto$ yield formulas not expressible in $\mathcal{E} \mathcal{L}^{++}$, and so the guarantee of tractability is lost.

\section{Example 46.}


Table 6

$\mathcal{E} \mathcal{L}^{++}$and Horn-SHOIQQ. The Horn-SHOIQ $\mathcal{S H o r m a l}$ form used is due to $43 . A, A_{1}, A_{2}, B$ are atomic concepts or nominals. $\mathcal{E} \mathcal{L}^{++}$ontologies may also contain RIAs.

\begin{tabular}{|c|c|c|}
\hline Language & GCIs & $\begin{array}{l}\text { Tractability } \\
\text { Preservation }\end{array}$ \\
\hline $\mathcal{E} \mathcal{L}^{++}$ & $\begin{array}{l}C \sqsubseteq D, \text { where } \\
C, D=\top|\perp|\{a\}\left|C_{1} \sqcap C_{2}\right| \exists r . C\end{array}$ & yes (only for $\sqsubset$ ) \\
\hline 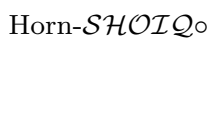 & $\begin{array}{l}\top \sqsubseteq A, A \sqsubseteq \perp, A_{1} \sqcap A_{2} \sqsubseteq B, \\
\exists R . A \sqsubseteq B, A \sqsubseteq \exists R . B, A \sqsubseteq \forall S . B, \\
A \sqsubseteq \geq n R . B, A \sqsubseteq \leq 1 R . B .\end{array}$ & yes (only for $\sqsubset$ ) \\
\hline
\end{tabular}

$$
\begin{array}{ll}
\pi\left(A_{1} \sqcap A_{2} \mapsto B\right)= & \neg\left(A_{1}^{\prime} \sqcup A_{2}^{\prime}\right) \sqsubseteq B \\
\pi\left(A_{1} \sqcap A_{2} \rightarrow B\right)= & \left\{A_{1} \sqcap A_{2} \sqsubseteq B,\right. \\
& \left.B^{\prime} \sqsubseteq\left(A_{1}^{\prime} \sqcup A_{2}^{\prime}\right)\right\}
\end{array}
$$

Given the above discussion, the following theorem holds.

Proposition 47. For any $\mathcal{E} \mathcal{L}^{++}$knowledge base $K B$ and axiom $\alpha, K B=_{4} \alpha$ iff $\pi(K B) \models_{2} \pi(\alpha)$. If all GCIs use $\sqsubset$, then $\pi(K B)$ is an $\mathcal{E} \mathcal{L}^{++}$knowledge base.

Proof. The first claim holds in virtue of Proposition 39. Because applying $\pi$ to any $\mathcal{E L}^{++}$concept, RIA, or GCI $C \sqsubset D$ yields an $\mathcal{E L}^{++}$expression, the second claim holds.

Furthermore, since there is no negative role constructor to cause further inconsistencies in $\mathcal{E} \mathcal{L}^{++}$, roles and RIAs can be interpreted classically $(\pi$ would not alter them, anyway).

\subsection{Horn-DLs}

We focus on Horn-SHOIQ 0 43. The conclusions obtained for it hold for other Horn-DLs as well, including Horn-SHOIQ [56]. It's assumed that Horn-SHOIQ $\circ$ ontologies are in the normal form 43 shown in Table 6

All of the Horn-SHOI $\mathcal{Q} \circ$ concept constructors result in Horn- $\mathcal{S H O I} \mathcal{I} \circ$ expressions under $\pi$ except $\leq 1 R . B$, because $\pi(\leq 1 R . B)=\leq 1 R . \neg B^{\prime}$. However, we can remain within Horn- $\mathcal{S H O \mathcal { I }} \circ$ by post-processing the result of $\pi$ as shown below.

$$
-\pi_{\text {Horn }}(C)=C \text {, if } C \text { is }
$$

$$
\top|\perp| A\left|A_{1} \sqcap A_{2}\right| \exists R . A|\forall S . B| \geq n R . B ;
$$

$$
\begin{aligned}
& -\pi_{\text {Horn }}(\leq 1 R \cdot B)=\leq 1 R \cdot B^{=} \text {(where } B^{=} \text {is a } \\
& \text { new concept }) \text { with } B^{=} \sqcap B^{\prime} \sqsubseteq \perp \text { asserted; } \\
& -\pi_{\text {Horn }}(\neg(\leq 1 R . B))=\geq 2 R . B .
\end{aligned}
$$

The last item is needed for processing inclusion axioms involving $<1 R$. B:

$$
\begin{aligned}
- & \pi_{\text {Horn }}(A \mapsto \leq 1 R \cdot B)= \\
& \left\{\neg A^{\prime} \sqsubseteq \leq 1 R \cdot B^{=}, B^{=} \sqcap B^{\prime} \sqsubseteq \perp\right\} \\
- & \pi_{\text {Horn }}(A \sqsubset \leq 1 R \cdot B)= \\
& \left\{A \sqsubseteq \leq 1 R \cdot B^{=}, B^{=} \sqcap B^{\prime} \sqsubseteq \perp\right\} \\
- & \pi_{\text {Horn }}(A \rightarrow \leq 1 R \cdot B)= \\
& \left\{A \sqsubseteq \leq 1 R \cdot B^{=}, B^{=} \sqcap B^{\prime} \sqsubseteq \perp, \geq 2 R . B \sqsubseteq A^{\prime}\right\}
\end{aligned}
$$

Given the above way of transforming axioms, $\pi_{\text {Horn }}(A \sqsubset \leq 1 R . B)$ is permissible, but the transformations for material and strong inclusion axioms are not. The example used for $\mathcal{E} \mathcal{L}^{++}$also works as a counterexample for the use of $\mapsto$ and $\rightarrow$ in Horn-SHOIQ $\circ$. Since $A_{1} \sqcap A_{2} \sqsubseteq B$ is allowed in other Horn-DLs, the same conclusion holds for them. And so, if we wish to preserve the structure of tractable Horn-DLs, we must use only internal inclusion.

Proposition 48. If $K B$ is a Horn-SHOIQ $\mathcal{S H n o w l - ~}$ edge base using only $\sqsubset$-axioms, then it follows that $\pi_{\text {Horn }}(\pi(K B))$ is a Horn-SHOIQ $\mathcal{S}$ knowledge base.

Given the revised $\pi$ function, the conclusion obviously holds. The revised function also works for DLP 28, which corresponds to OWL 2 RL.

Observe that the additional transformations are sound, in the following sense.

Proposition 49. Let $K B \cup\{A\}$ be a set of Horn$\mathcal{S H O I \mathcal { Q }}$ assertions, with all GCIs being $\sqsubset-$ axioms. If $\pi_{\text {Horn }}(\pi(K B))={ }_{2} \pi_{\text {Horn }}(\pi(A))$, then $\pi(K B)=_{2} \pi(A)$. 


\subsection{DL-Lite}

The logics of the DL-Lite family are the maximal DLs supporting efficient query answering over large numbers of instances. The usual DL reasoning tasks performed in the DL-Lite family are known to be polynomial in the size of the KB (and LOGSPACE in the size of the ABox), and query answering is LogSpace in the size of the ABox [12]. Moreover, the DL-Lite family allows for separation between TBox and ABox reasoning during query evaluation. In particular, ABox reasoning can be carried out by an SQL engine 12 .

We focus on two members of the DL-Lite family: DL-Lite $_{\text {core }}$, which forms the kernel of the family; and DL-Lite $\mathcal{R}_{\mathcal{R}}$, which corresponds to OWL 2 QL.

Concepts and roles of the DL-Lite family are formed using the grammar 12

$$
\begin{array}{ll}
B::=A \mid \exists R & R::=P \mid P^{-} \\
C::=B \mid \neg B & E::=R \mid \neg R
\end{array}
$$

where $A \in N_{C}, P \in N_{R}$, and $P^{-} \in N_{R}^{-}$. Table 7 shows the syntax definitions of GCIs and RIAs.

A paraconsistent semantics similar to that described for $\mathcal{S R O I Q}$ can be provided for DLLite $_{\text {core }}$ and DL-Lite $\mathcal{R}_{\mathcal{R}}$. Roles are 4-valued (Table 8), but $\exists R$ and $\neg \exists R$ are treated differently than in $\mathcal{S R O} \mathcal{I} \mathcal{Q} 4$. Intuitively, $p^{+}\left((\exists R)^{\mathcal{I}}\right)$ is set of individuals $x$ positively related to some $y$ via $R$, while $p^{-}\left((\exists R)^{\mathcal{I}}\right)$ is the set of individuals $x$ negatively related to all $y$ via $R$.

The knowledge base $\{B \sqsubseteq \neg A, B(a), A(a)\}$ is classically unsatisfiable in DL-Lite core $_{\text {c }}\left\{P_{1} \sqsubseteq\right.$ $\left.P_{2}, P_{1} \sqsubseteq \neg P_{2}, P_{1}(a, b)\right\}$ is classically unsatisfiable in DL-Lite ${ }_{\mathcal{R}}$. In the 4 -valued context, however, every knowledge base is satisfiable.

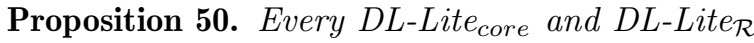
knowledge base is 4-satisfiable.

The DL-Lite $\pi$-transformations are given below. It is clear that the transformation of internal inclusion axioms stays within DL-Lite.

Definition 51. Where $R$ and $E$ are roles, the $D L$ Lite $\pi$ transformations are defined inductively.

$-\pi_{\text {Lite }}(R \sqsubseteq E)=\pi_{\text {Lite }}(R) \sqsubseteq \pi_{\text {Lite }}(E)$;

- $\pi_{\text {Lite }}(\neg R)=R^{\prime}$, where $R^{\prime}$ is a new role name;

- $\pi_{\text {Lite }}(\exists R)=\exists R$;

- $\pi_{\text {Lite }}(\neg \exists R)=\neg \exists R^{=}$, where $R^{=}$is a new role name and $R^{=} \sqsubseteq \neg R^{\prime}$;
For material inclusion and strong inclusion, because negated concepts are not allowed to occur on the left of a GCI, they do not preserve the DLLite structure. In contrast, the transformation of internal inclusion remains within DL-Lite. However, observe that the transformation

$$
\pi_{\text {Lite }}(A \sqsubset \neg \exists R)=\left\{A \sqsubseteq \neg \exists R^{=}, R^{=} \sqsubseteq \neg R^{\prime}\right\}
$$

introduces a role inclusion axiom. In general, a DLLite $_{\text {core }}$ knowledge base might be transformed into a DL-Lite $\mathcal{R}_{\mathcal{R}}$ knowledge base. Regardless of this, the transformation preserves consequences.

Proposition 52. If $K B$ is a DL-Lite knowledge base and $A$ an axiom (in which neither $\mapsto$ nor $\rightarrow$ are used),

$$
K B=_{4} A \text { iff } \pi_{\text {Lite }}(K B) \models_{D L \text {-Lite }} \pi_{\text {Lite }}(A) .
$$

\section{ELP}

ELP 44 is a tractable rule language combining features of $\mathcal{E} \mathcal{L}^{++}$with Horn-like rules. Syntactically, ELP resembles Datalog or logic programming, in the sense that well-formed formulas are rules of the form $B \mapsto H$, where $B$ and $H$ are both conjunctions. In ELP, however, each conjunct is either a concept atom $C(t)$ or a role atom $R\left(t_{1}, t_{2}\right)$, where $C$ is an $\mathcal{E} \mathcal{L}^{++}$concept description, $R$ is an $\mathcal{E} \mathcal{L}^{++}$role, and each $t$ is a term (an individual from $N_{I}$ or else a variable from a set $V$ of variables). Each rule is implicitly universally quantified (there are no free variables). To ensure decidability, it is assumed that there is a fixed set $V_{s} \subseteq V$ of safe variables; these range only over named elements of the domain of discourse.

Below, we present a paraconsistent semantics for ELP, defining what we call ELP4. The semantics is similar to one provided for a first-order logic defined by Lang [45], the primary differences being that we make use of internal implication $\supset$ where Lang uses his own implication operator, and we include safe variables (these could be omitted, however, yielding an undecidable logic). The rules described below confine themselves to what are essentially unary and binary predicates, but it is clear that the semantics can be easily modified to apply to rules using predicates of arbitrary arity. Furthermore, in order to ensure tractability, ELP rules conform to certain syntactic restrictions. We 
Table 7

DL-Lite Family

\begin{tabular}{l|c|c|c} 
Language & GCIs & Role Inclusions & Tractability preservation \\
\hline \hline DL-Lite $_{\text {core }}$ & $B \sqsubseteq C$ & $\varnothing$ & yes (only for $\sqsubset$ ) \\
\hline DL-Lite $_{\mathcal{R}}$ & $B \sqsubseteq C$ & $R \sqsubseteq E$ & yes (only for $\sqsubset$ )
\end{tabular}

Table 8

Four-valued semantics for expressions in DL-Lite

\begin{tabular}{c|c} 
Syntax & Semantics \\
\hline$\exists R$ & $\left\langle\left\{x \mid \exists y,(x, y) \in p^{+}\left(R^{\mathcal{I}}\right)\right\},\left\{x \mid \forall y,(x, y) \in p^{-}\left(R^{\mathcal{I}}\right)\right\}\right\rangle$ \\
$\neg \exists R$ & $\left\langle\left\{x \mid \forall y,(x, y) \in p^{-}\left(R^{\mathcal{I}}\right)\right\},\left\{x \mid \exists y,(x, y) \in p^{+}\left(R^{\mathcal{I}}\right)\right\}\right\rangle$
\end{tabular}

do not discuss the restrictions below. However, the embedding into classical ELP maintains the syntactic structure of the rules, and so tractability is preserved. Also, since the restrictions are not part of the discussion, it is clear that most of the definitions and results below apply equally well to rule-bases that do not satisfy them.

As originally defined, a rule base for ELP consists of rules of the form $B \mapsto H$, where $\mapsto$ is just material implication. In moving to the 4 -valued context, we also allow rules using internal $(\supset)$ and strong $(\rightarrow)$ implication. 4-valued interpretations for ELP rule bases are defined essentially as they were earlier. However, semantics for rules must be given. This is done by defining two forms of satisfaction: t-satisfaction $\left(\left.\right|_{t}\right)$ and f-satisfaction $\left(\left.\right|_{f}\right)$. The notation $\models_{t}$ and $\models_{f}$ is adopted from Lang 45.

Definition 53. An element $d \in \Delta^{\mathcal{I}}$ is named in interpretation $\mathcal{I}$ iff there is an $a \in N_{I}$ such that $a^{\mathcal{I}}=d$. Otherwise, $d$ is unnamed in $\mathcal{I}$. A variable assignment $\sigma_{\mathcal{I}}$ for $\mathcal{I}$ is a function from $V \cup N_{I}$ to $\Delta^{\mathcal{I}}$ such that

1. if $t \in N_{I}$, then $\sigma_{\mathcal{I}}(t)=t^{\mathcal{I}}$;

2. if $t \in V_{S}$, then $\sigma_{\mathcal{I}}(t)$ is named in $\mathcal{I}$.

If $\mathcal{I}$ is an interpretation and $\sigma_{\mathcal{I}}$ an assignment for $\mathcal{I}$, then $\sigma_{\mathcal{I}}[x / d]$ is a function from $V \cup N_{I}$ to $\Delta^{\mathcal{I}}$ such that for all $t \in N_{I} \cup V$,

1. if $t \neq x$, then $\sigma_{\mathcal{I}}[x / d](t)=\sigma_{\mathcal{I}}(t)$;

2. otherwise, $\sigma_{\mathcal{I}}[x / d](t)=d$.

Observe that if $x \in V_{s}$ but $d$ is not named in $\mathcal{I}$, $\sigma_{\mathcal{I}}[x / d]$ is not a valid assignment.

Definition 54. Let $\mathcal{I}$ be an interpretation and $\sigma_{\mathcal{I}}$ a variable assignment for $\mathcal{I}$.

1. If $C$ is a concept description and $t$ a term,
$-\mathcal{I}, \sigma_{\mathcal{I}} \models_{t} C(t)$ iff $\sigma_{\mathcal{I}}(t) \in p^{+}\left(C^{\mathcal{I}}\right)$.

$-\mathcal{I}, \sigma_{\mathcal{I}} \models_{f} C(t)$ iff $\sigma_{\mathcal{I}}(t) \in p^{-}\left(C^{\mathcal{I}}\right)$.

2. If $R$ is a role and $t_{1}$ and $t_{2}$ terms,

$-\mathcal{I}, \sigma_{\mathcal{I}} \models_{t} R\left(t_{1}, t_{2}\right)$ iff $\left(\sigma_{\mathcal{I}}\left(t_{1}\right), \sigma_{\mathcal{I}}\left(t_{2}\right)\right) \in$ $p^{+}\left(R^{\mathcal{I}}\right)$.

$-\mathcal{I}, \sigma_{\mathcal{I}} \models_{f} R\left(t_{1}, t_{2}\right)$ iff $\left(\sigma_{\mathcal{I}}\left(t_{1}\right), \sigma_{\mathcal{I}}\left(t_{2}\right)\right) \in$ $p^{-}\left(R^{\mathcal{I}}\right)$.

3. For conjunction $(\mathcal{P} \wedge \mathcal{Q})$,

$-\mathcal{I}, \sigma_{\mathcal{I}} \models_{t}(\mathcal{P} \wedge \mathcal{Q})$ iff $\mathcal{I}, \sigma_{\mathcal{I}}=_{t} \mathcal{P}$ and $\mathcal{I}, \sigma \models_{t} \mathcal{Q}$,

$-\mathcal{I}, \sigma_{\mathcal{I}} \models_{f}(\mathcal{P} \wedge \mathcal{Q})$ iff $\mathcal{I}, \sigma_{\mathcal{I}} \models_{f} \mathcal{P}$ or $\mathcal{I}, \sigma \models_{f} \mathcal{Q}$,

4. For material implication $(\mathcal{P} \mapsto \mathcal{Q})$,

$-\mathcal{I}, \sigma_{\mathcal{I}} \models_{t}(\mathcal{P} \mapsto \mathcal{Q})$ iff $\mathcal{I}, \sigma_{\mathcal{I}} \models_{f} \quad \mathcal{P}$ or $\mathcal{I}, \sigma_{\mathcal{I}} \models_{t} \mathcal{Q}$,

$-\mathcal{I}, \sigma_{\mathcal{I}} \models_{f}(\mathcal{P} \mapsto \mathcal{Q})$ iff $\mathcal{I}, \sigma_{\mathcal{I}} \models_{t} \mathcal{P}$ and $\mathcal{I}, \sigma_{\mathcal{I}} \models_{f} \mathcal{Q}$,

5. For internal implication $(\mathcal{P} \supset \mathcal{Q})$,

$-\mathcal{I}, \sigma_{\mathcal{I}}=_{t}(\mathcal{P} \supset \mathcal{Q})$ iff $\mathcal{I}, \sigma_{\mathcal{I}} \forall_{t} \mathcal{P}$ or $\mathcal{I}, \sigma_{\mathcal{I}} \models_{t} \mathcal{Q}$,

$-\mathcal{I}, \sigma_{\mathcal{I}} \models_{f}(\mathcal{P} \supset \mathcal{Q})$ iff $\mathcal{I},\left.\sigma_{\mathcal{I}}\right|_{t} \mathcal{P}$ and $\mathcal{I}, \sigma_{\mathcal{I}} \models_{f} \mathcal{Q}$,

6. For strong implication $(\mathcal{P} \rightarrow \mathcal{Q})$,

$-\mathcal{I}, \sigma_{\mathcal{I}}=_{t}(\mathcal{P} \rightarrow \mathcal{Q})$ iff $\left(\mathcal{I}, \sigma_{\mathcal{I}} \nvdash_{t} \quad \mathcal{P}\right.$ or $\left.\mathcal{I}, \sigma_{\mathcal{I}} \models_{t} \mathcal{Q}\right)$ and $\left(\mathcal{I}, \sigma_{\mathcal{I}} \models_{f} \mathcal{P}\right.$ or $\left.\mathcal{I}, \sigma_{\mathcal{I}} \not{ }_{f} \mathcal{Q}\right)$.

$-\mathcal{I}, \sigma_{\mathcal{I}} \models_{f}(\mathcal{P} \rightarrow \mathcal{Q})$ iff $\mathcal{I}, \sigma_{\mathcal{I}} \models_{t} \mathcal{P}$ and $\mathcal{I}, \sigma_{\mathcal{I}} \models_{f} \mathcal{Q}$.

7. For $(\forall x) \mathcal{P}$,

(a) if $x \in V_{s}$,

$-\mathcal{I}, \sigma_{\mathcal{I}} \models_{t}(\forall x) \mathcal{P}$ iff $\mathcal{I}, \sigma_{\mathcal{I}}[x / d] \models_{t} \mathcal{P}$ for all named $d \in \Delta^{\mathcal{I}}$,

- $\mathcal{I},\left.\sigma_{\mathcal{I}}\right|_{f}(\forall x) \mathcal{P}$ iff $\mathcal{I},\left.\sigma_{\mathcal{I}}[x / d]\right|_{f} \mathcal{P}$ for some named $d \in \Delta^{\mathcal{I}}$.

(b) if $x \notin V_{s}$, 


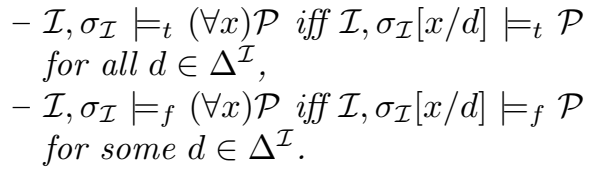

Definition 55. Let $\mathcal{I}$ be an interpretation, $\mathcal{P}$ a wellformed formula of $E L P$, and $K$ a set of such formulas.

1. $\mathcal{I}$ 4-satisfies $\mathcal{P}$ ( $\mathcal{I}$ is a 4-model of $\mathcal{P})$ iff $\mathcal{I}, \sigma_{\mathcal{I}} \models_{t} \mathcal{P}$ for each assignment $\sigma_{\mathcal{I}}$.

2. $K$ entails $\mathcal{P}\left(K \models_{E L P 4} \mathcal{P}\right)$ iff every 4-model of $K$ is a 4 -model of $\mathcal{P}$.

According to 44, every classical ELP rule base can be converted into an equisatisfiable one via the transformation rules shown in Table 9 Since there are three different types of rules, the analogous claim is problematic in the 4 -valued context. Nevertheless, if rules are interpreted using $\supset$, conversion to normal form preserves 4-satsifiability.

Definition 56. Let $R B$ be an ELP rule base utilizing only $\supset$-rules. $R B$ is in normal form iff for each rule $B \supset H$,

1. Each concept atom in $B$ is of the form $C(t)$, where $C \in N_{C} \cup N_{o} \cup\{\top\}$,

2. Each atom in $H$ is of the form $A(t), \exists R . B(t)$, or $R(t, u)$, where
(a) $A \in N_{C} \cup N_{o} \cup\{\perp\}$,
(b) $B \in N_{C}$,
(c) $R \in N_{R}$, and
(d) $t, u \in V \cup N_{I}$.

3. Every variable in $H$ appears in $B$.

Proposition 57. Let $K B$ be a $\supset$-rule base and $K B^{\prime}$ the result of applying one of $H 1, \ldots, H 4, B 1, \ldots, B 3$ from Table 9 to KB. KB is 4-satisfiable iff $K B^{\prime}$ is.

We define the sequence $K B_{0}, K B_{1}, \ldots$, where $K B_{0}=K B$ and $K B_{n+1}$ is the result of applying one transformation rule to $K B_{n}$. Let $K B_{N}$ be the first $n$ such that $K B_{n}=K B_{n+1} . K B_{N}$ is in normal form [44. Given the above proposition, it is clear that $K B_{N}$ is satisfiable if and only if $K B$ is.

The transformation $\pi$ can be extended in a rather simple fashion to embed ELP4 into classical ELP, provided $\supset$ is used. As with the tractable description logics, the other inclusion operators yield structures outside of the language (one could nevertheless use $\rightarrow$ and $\mapsto$ as well, translating them as indicated in Table 5 . Doing so would not yield meaningless expressions, but the results would not constitute expressions of ELP). Since the structure of the rule base is left intact by the transformation, the syntactic constraints on ELP rules are met.

$$
\begin{aligned}
& \pi(C(t))=\pi(C)(t) \\
& \pi\left(R\left(t_{1}, t_{2}\right)\right)=\pi(R)\left(t_{1}, t_{2}\right) \\
& \pi(\mathcal{Q} \wedge \mathcal{R})=\pi(\mathcal{Q}) \wedge \pi(\mathcal{R}) \\
& \pi(\mathcal{Q} \vee \mathcal{R})=\pi(\mathcal{Q}) \vee \pi(\mathcal{R}) \\
& \pi(\mathcal{Q} \supset \mathcal{R})=\pi(\mathcal{Q}) \mapsto \pi(\mathcal{R}) \\
& \pi((\forall x) \mathcal{Q})=(\forall x) \pi(\mathcal{Q})
\end{aligned}
$$

Define $\pi(K B)$ as $\{\pi(R) \mid R \in K B\}$. Earlier, we defined the primed counterpart $\mathcal{I}^{\prime}$ for each 4 -interpretation $\mathcal{I}$, and we showed that for any $\mathcal{S R O I} \mathcal{Q}$ concept $C, p^{+}\left(C^{\mathcal{I}}\right)=\pi(C)^{\mathcal{I}^{\prime}}$ and $p^{-}\left(C^{\mathcal{I}}\right)=\pi(\neg C)^{\mathcal{I}^{\prime}}$. We show here that for any rule $r: B \supset H, \mathcal{I}$ 4-satisfies $r$ iff $\mathcal{I}^{\prime}$ 2-satisfies $\pi(r) 5^{5}$

Proposition 58. If $\mathcal{P}$ is a well-formed formula of $E L P, \mathcal{I}$ is a 4-interpretation of $\mathcal{P}$, and $\mathcal{I}^{\prime}$ is the primed counterpart of $\mathcal{I}$, then for any assignment $\sigma_{\mathcal{I}}, \mathcal{I}, \sigma_{\mathcal{I}} \models_{t} \mathcal{P}$ iff $\mathcal{I}^{\prime}, \sigma_{\mathcal{I}^{\prime}}=\pi(\mathcal{P})$.

From this, the preservation of consequences readily follows.

Proposition 59. Let $K B$ be an ELP rule base and $\mathcal{P}$ a well-formed formula of ELP. KB $\models_{E L P 4} \mathcal{P}$ iff $\pi(K B) \models_{E L P} \pi(\mathcal{P})$.

Example 60. The following set $R B$ of (normal form) ELP rules is classically inconsistent.

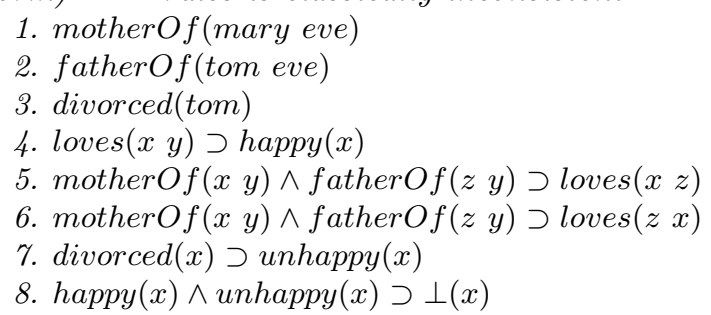

Because of the occurrence of $\perp$, it is also inconsistent under the 4-valued semantics. This can be remedied by replacing rule 8 with $8^{\prime}$ (to form $R B^{\prime}$ ).

8.' happy $(x) \wedge$ unhappy $(x) \supset A_{\perp}(x) \wedge \neg A_{\perp}(x)$.

Though the negation is not technically allowed in $\mathcal{E} \mathcal{L}^{++}$, Upon transformation by $\pi, A_{\perp}(x) \wedge \neg A_{\perp}(x)$ becomes $A_{\perp}(x) \wedge A_{\perp}^{\prime}(x)$, which is allowed. Apart

${ }^{5}$ Observe that the set of variables is fixed, regardless of whether one is talking about $\mathcal{I}$ or $\mathcal{I}^{\prime}$; furthermore, the interpretation of individuals and variables is the same in both interpretations. And so "safety" is not affected. 
Table 9

Converting a classical ELP rule $B \mapsto H$ to normal form (taken from [4]).

\begin{tabular}{|l|l|}
\hline H1 & If $(C \sqcap D)(t) \in H$, then replace it with $C(t) \wedge D(t)$. \\
H2 & If $\top(x) \in H$, then delete it. If $H$ would then be empty, delete $r$ from $K B$. \\
H3 & If $H$ contains a variable $x$ not occurring in $B$, add $\top(x)$ to $B$. \\
H4 & If $\exists R . C(t) \in H$ with $C \notin N_{C}$, replace it in $r$ with $\exists R . A(t)$ where $A$ is new to $K B$, and add \\
B1 & the rule $A(x) \mapsto C(x)$. \\
B2 & If $\perp \sqcap D)(t) \in B$, then replace it with $C(t) \wedge D(t)$. \\
B3 & If $\exists R . C(t) \in B$, delete $r$ from $K B$. \\
\hline
\end{tabular}

from this and the replacement of $\supset$ with $\mapsto$, the rule base is otherwise unaltered 6

We note that $R B^{\prime}$ is 4-satisfiable. Furthermore, $\pi\left(R B^{\prime}\right)$ is 2-satisfiable. In both, one may infer happy(tom) and unhappy(tom) (under the appropriate semantics). However, in neither case can one infer an arbitrary expression such as unhappy (mary).

If one were to attempt to transform the rule base using strong implication (according to Table 5), then rules 5, 6, and 8 would introduce disjunctions. For instance,

$5_{1}^{\prime}$ motherOf $(x y) \wedge$ father $O f(z y) \mapsto \operatorname{loves}(x z)$ $5_{2}^{\prime}$ loves $^{\prime}(x z) \mapsto$ motherOf $f^{\prime}(x y) \vee$ fatherO $^{\prime}(z y)$.

This is outside of the rule language.

\section{Empirical Evaluations}

The last two sections indicate that, for some description logics and their rule-based extensions, internal inclusion must be used in order to guarantee tractability. However, even in cases where the translation does not lead to expressions of a strictly more expressive language - e.g., as in the case of $\mathcal{S R O I Q ~ - ~ t h e ~ c h o i c e ~ o f ~ o p e r a t o r ~ c a n ~ h a v e ~}$ a significant effect on performance. While embedding internal inclusion axioms into a classical DL potentially leads to the creation of new concept names, it typically does not increase the number of inclusion axioms 7 and the translation elimi-

\footnotetext{
${ }^{6}$ Though not explicitly stated in 44, A rule of the form $B \mapsto C(t) \wedge D(t) \wedge \ldots$ can be split into two rules, $B \mapsto C(t) \wedge \ldots$ and $B \mapsto D(t) \wedge \ldots$. We will assume that such transformations are also performed when converting to normal form.

${ }^{7}$ This is not true for the paraconsistent versions of Horn DLs and DL-Lite, as discussed earlier; for those logics, new axioms are added.
}

nates all occurrences of negation. In contrast, embedding strong inclusion axioms essentially doubles both the number of concept names and the number of inclusion axioms. Embedding material inclusion introduces negation into the left-handsides of axioms, and it potentially leads to the introduction of a greater number of new concepts than does internal inclusion. Though the size of the translation is linear with regard to the size of the original regardless of the inclusion operator used, these differences can have a significant impact and make reasoning with strong and material inclusion more difficult than reasoning with internal inclusion alone.

We have performed a set of empirical tests which, though tentative and limited in nature, do appear to support this claim. Several knowledge bases (either downloaded or else programmatically created) were translated according to the $\pi$ function described in Section 5, and a DL reasoner (Pellet 67]) was used to process both the original knowledge bases and their translations. Specifically, the reasoner computed class and property hierarchies, determined class membership for individuals, and checked for consistency. Some but not all of the original ontologies were satisfiable 8

The translations were performed using a custom Java library implementing the $\pi$ function and based on methods provided by the OWL API 33 . Three distinct translations of each knowledge base were created, each interpreting the inclusion axioms of the original according to one of the three new operators.

Two knowledge bases were created specifically for the tests using the Lehigh Benchmark (LUBM)

\footnotetext{
${ }^{8}$ The number of unsatisfiable concepts in each ontology is: amino-acid (0), pizza (2), proton (15), tambis-patched (144), university-1 (0), university-2 (0), wine (0).
} 
suite [27]. The suite provides a tool for generating ontologies populated with a variable number of universities, departments, professors, students, etc. For the evaluations, the default settings of the tool were used, but the resulting knowledge bases were manually edited $\sqrt{9}$ Disjoint class axioms were also manually added (asserting, e.g., that assistant, associate, and full professors are disjoint classes). Inconsistent versions of the knowledge bases were also created by inserting same-as assertions (stating, for instance, that a particular student was identical to a publication).

For the experiments, each ontology was processed 21 times (without restarting between runs), and the time required to complete all computations was recorded. The first run-which involved memory allocation not occurring in subsequent runs - was ignored in each case. A 15 minute timelimit was used; runs requiring more time than that were terminated. All experiments were performed on a consumer desktop computer.

Tables 10 and 11 describe the knowledge bases evaluated. The relative sizes of the translations are given in Table 12. As shown there, translating according to strong inclusion typically increases the number of concept names by a factor of 2 . The number of inclusion axioms is not doubled - it is assumed that this is due to the presence of duplicate axioms that are eliminated by the OWL API. As far as the concepts are concerned, factors greater than 2 can be attributed to the presence of nominals in the input. Factors less than 2 are also possible, as when concepts only appear in positive concept assertions. Using material inclusion or internal inclusion also increases the number of concepts. However, this number is smaller than when strong inclusion is used.

Table 13 displays the average time required to process the knowledge bases and their translations. When internal inclusion was used, the test system was in each case able to process the translation (the time required was significantly greater than that needed for the original, however). In

\footnotetext{
${ }^{9}$ The smaller knowledge base consisted of 5 departments, 25 research groups, 1000 (graduate and teaching) courses, 100 professors (lecturer, assistant, associate, full), 1000 students (graduate and undergraduate), 500 publications, and 200 teaching and research assistants. The larger instance contained roughly 2-8 times these numbers. In both cases, 10 universities were used.
}

contrast, if either strong or material inclusion was used, processing the translation was typically not possible. The reasoner either ran out of memory, or else execution was manually terminated due to the time-limit. The inability to process knowledge bases based on material inclusion can be explained by the introduction of negation into axioms. This does not occur when internal inclusion is used.

\section{Conclusions and Related Work}

In this paper, we have described several para-

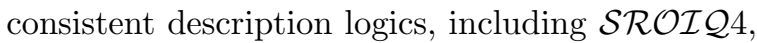
and we have shown that they are classically sound and that their embeddings into classical logics are consequence preserving. It should be pointed out that since the most recent version of OWL [30] is

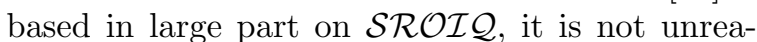
sonable to suppose that $\mathcal{S R O \mathcal { I }} 4$ has real practical value. Inconsistencies often arise in real world situations, and the theory surrounding $\mathcal{S R O I Q 4}$ allows the use of existing classical OWL inference engines to reason correctly even over inconsistent OWL knowledge bases.

The issue of handling inconsistency is a common one in the field of knowledge representation and reasoning, and also more generally in the context of data management. Beyond multivalued paraconsistent logics, several other approaches have been studied. For instance, regardless of whether they are paraconsistent or not, the various nonmonotonic logics that have been developed over the years (e.g., Reiter's default logic 63], logic programming under the answer-set [25] or wellfounded [26] semantics, defeasible logic [58]) can be viewed at least in part as attempts to resolve conflicts between assertions. E.g., the extended logic program

$$
\begin{aligned}
p & :-\sim \neg p \\
\neg p & :-\sim p \\
q & :-p \\
q & :-\neg p
\end{aligned}
$$

possesses two answer sets $(\{p, q\}$, and $\{\neg p, q\})$, and $q$ is considered a consequence of the program. The first two rules can be seen as asserting presumptive (and conflicting) evidence for both $p$ and 
Table 10

Test knowledge bases

Name

Description

Amino Acid

An $\mathcal{A L C F}(D)$ ontology pertaining to amino-acids. http://www.co-ode.org/ontologies/ amino-acid/2006/05/18/amino-acid.owl

Pizza

A $\mathcal{S H O I N}$ ontology making frequent use of $\exists$ - and $\forall$-restrictions, and infrequent use of nominals. http://www.co-ode.org/ontologies/pizza/2007/02/12/

Proton A $\mathcal{S H \mathcal { I }}(D)$ ontology using concepts from the PROTON ontologies, with disjointness axioms added. http://proton. semanticweb.org/

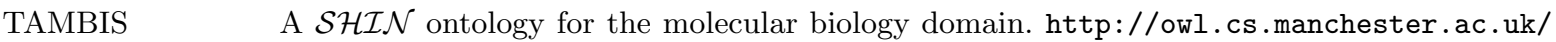
repository/ (tambis-patched.owl)

Wine A $\mathcal{S H O \mathcal { H }}(D)$ ontology making heavy use of nominals. http://www.w3.org/TR/ owl-guide/wine.rdf

University $1 \quad$ A $\mathcal{A} \mathcal{L} \mathcal{E} \mathcal{H} \mathcal{I}+(D)$ ontology made using the Lehigh Ontology Benchmark Suite [27. DisjointClasses axioms were manually added. A separate inconsistent version was created by adding SameAs assertions for individuals in disjoint classes. http://swat.cse.lehigh.edu/ projects/lubm/

University 2 A larger version of University 1. An inconsistent version of the ontology was also generated.

Table 11

Knowledge base sizes

\begin{tabular}{lccccccc} 
KB & Concepts & Ind. & GCIs & Concept Assert. & (Obj) Role Assert. & Disjoint Concepts & Equiv Concepts \\
\hline Amino Acid & 46 & 0 & 238 & 0 & 0 & 199 & 12 \\
Pizza & 100 & 5 & 259 & 10 & 0 & 0 & 1298 \\
Proton & 266 & 0 & 278 & 0 & 0 & 0 & 21 \\
TAMBIS & 395 & 0 & 343 & 182 & 246 & 11233 & 1 \\
Wine & 77 & 161 & 126 & 2840 & 41095 & 61 \\
University 1 & 43 & 2640 & 36 & 14665 & & 6 \\
University 2 & 43 & 13865 & 36 & & & & 6
\end{tabular}

Table 12

Size increase (relative to original)

Concept GCIs

\begin{tabular}{lcccccc} 
Ontology & Internal & Strong & Material & Internal & Strong & Material \\
\hline Amino Acid & 1.7 & 2 & 1.98 & 1 & 1.84 & 1 \\
Pizza & 1.77 & 2.03 & 1.75 & 1 & 1.27 & 1 \\
Proton & 1.99 & 2 & 1.39 & 1 & 1.1 & 1 \\
TAMBIS & 1.07 & 2 & 1.72 & 1 & 1.94 & 1 \\
Wine & 1.03 & 2.82 & 2.53 & 1 & 1.99 & 1 \\
University 1 & 1.51 & 2 & 1.72 & 1 & 1.43 & 1 \\
University 2 & 1.51 & 2 & 1.72 & 1 & 1.43 & 1 \\
\hline
\end{tabular}

Disjoint class axioms and equivalent class axioms

were interpreted as sets of inclusion axioms. 
Table 13

Average classification times, in milliseconds.

\begin{tabular}{lcccc} 
Knowledge Base & Original & Internal & Strong & Material \\
\hline Amino Acid & 102 & 236 & time-limit & 18800 \\
Pizza & 344 & 364 & time-limit & mem-limit \\
Proton & 121 & 592 & 739 & 302850 \\
TAMBIS & 337 & 1098 & mem-limit & mem-limit \\
Wine & 23105 & 84201 & time-limit & time-limit \\
University 1 & 251 & 4415 & time-limit & mem-limit \\
University 1 (Inconsistent) & N/A & 4838 & time-limit & mem-limit \\
University 2 & 1066 & 58542 & time-limit & mem-limit \\
University 2 (Inconsistent) & N/A & 58805 & time-limit & mem-limit \\
\hline Computer: AMD Athlon II X2, 6GB DDR2 RAM, Java SE 6 (64-bit, 4GB RAM). \\
time-limit: program was manually terminated after 15 minutes. \\
mem-limit: program exceeded available memory (and automatically terminated).
\end{tabular}

$\neg p$, and the conflict is resolved by disallowing both $p$ and $\neg p$ from appearing in a common answer set. The answer-set semantics, like Reiter's default logic and the other common semantics for logic programs, espouses the principle of explosion. The mechanism by which conflict is handled is default negation, and there are programs that are inherently inconsistent (regardless of whether default negation appears); drawing reasonable conclusions from such programs is impossible under the most common semantics for them. Nevertheless, alternative paraconsistent semantics for logic programs 19 207172 , and for default logic 297374, have been proposed.

The most obvious distinction between such formalisms and the paraconsistent logics presented here is that the former are nonmonotonic whereas the latter (like the underlying description logics upon which they are based) are monotonic. While nonmonotonicity is justifiable in many cases (arguably, it is required if one is to model certain forms of reasoning), it typically comes with a computational penalty. Unrestricted default logic is not recursively enumerable, for instance. Furthermore, it would be difficult to embed an inherently nonmonotonic logic into a monotonic one, which is what we have done here with the paraconsistent description logics.

Even within the paraconsistent reasoning community, there are approaches for achieving paraconsistency that differ from the multivalued approach we have described here. Jaśkowski's 23 38 discussive or discursive logics (or "logics of discussion") are considered to the first paraconsistent logic in modern times. In such logics, a proposition may be viewed as true whenever it has been proposed by a participant in a discussion. Since it is possible for participants to disagree, statements can be both true and false. The semantics for discussive logics are typically specified indirectly by a modal logic (e.g., S5). A sentence $\mathcal{P}$ of the theory, called a discussive assertion, would be treated as $\diamond \mathcal{P}$ ("possibly $\mathcal{P}$ ") and interpreted according to the modal logic. As in the multivalued logics presented here, many common rules of inference do not naturally hold under this interpretation. In particular, Modus Ponens fails, as does the Rule of Adjunction $(\{\mathcal{P}, \mathcal{Q}\} / \therefore \mathcal{P} \wedge \mathcal{Q})$. Jaśkowski thus developed both a "discussive implication" and a "discussive conjunction".

Newton da Costa was the first to develop and publish first-order paraconsistent logics in the 1950 s and 1960s, and for that reason he is often considered the father of paraconsistency 1618. Jaśkowski's work was confined to the propositional level and was not widely known at the time [17. Da Costa is best known for his $\mathcal{C}$-systems, which constitute an infinite hierarchy of paraconsistent logics. Their defining characteristic is that consistency is explicitly represented in the object language itself. I.e., a new unary operator $\circ$ is added, with $\circ \mathcal{P}$ stating that $\mathcal{P}$ is consistent. The logics are typically defined axiomatically, avoiding explosion by replacing certain classical axioms with weakened counterparts. Semantically, the logics break the truth-functional connection between a sentence and its negation. Much later, Carnielli, Coniglio, and Marcos would generalize 
the $\mathcal{C}$-systems, defining the logics of formal inconsistency [1415].

Neither discussive logic nor the logics based on da Costa's have in our opinion received much coverage in fields related to computer science. Within the philosophical and mathematical communities, discussive logic has received quite a bit of criticism, particularly for the failure of Adjunction. In our view, however, many critics appear simply to be unaware of Jaśkowski's work on discussive conjunction. Both sorts of logic have received criticism for breaking the usual behavior of negation, conjunction, and disjunction (e.g., in at least some of $\mathcal{C}$-systems, De Morgan's laws do not hold). One of the few frameworks utilizing a logic of formal inconsistency in an information system is described in 21. There, ideas from the stable model and well-founded semantics for logic programs are combined to create a paraconsistent version of Datalog.

The formalisms we have noted above allow an assertion and its negation to be simultaneously true (in some sense), and the formalisms permit reasoning under these conditions. In a way, such systems can be viewed as tolerating inconsistency. There are indeed a great many other approaches to handling inconsistency (see, e.g., the papers found in 11]). In one common view, inconsistencies in a knowledge base are taken as errors, and algorithms are developed to identify the errors and repair the knowledge base. Classic examples of this would be Reiter's work 64 on fault diagnosis and the wide body of literature devoted to belief revision and truth maintenance systems 12224 . Other work focuses on the identification of answers to queries that are consistent with repaired versions of inconsistent knowledge bases (regardless of whether or not the knowledge base actually has been repaired) 213. We note that the view underlying such work -i.e., that inconsistencies are faults or errors in the system - is often correct, and in such cases it is important to identify the sources of the error. This is something that the multivalued approach described here does not do. In the semantic web context, work on ontology debugging and determining justifications in ontologies (e.g., [40 75]) may also be considered relevant.

Returning to multivalued logics, the logics presented in this paper are related to earlier 4-valued description logics developed by Patel-Schneider 5960] and also Straccia, Sebastiani, and Meghini
[54556869]. $\mathcal{S R O I Q 4}$ is similar to these in the sense that all are 4-valued and essentially based on the logics of Belnap, but the earlier logics correspond neither syntactically nor semantically to $\mathcal{S R O I}$ Q4. The earlier logics are in fact syntactically closer to $\mathcal{A L C}$ and $\mathcal{S H \mathcal { L }}$, but there are differences even when restricted to these. PatelSchneider's logic does not allow concept union or existential role restrictions, and cardinality restrictions are unqualified. Equality in the logic is nonclassical-specifically, it is 4-valued, reflexive, and symmetric, but it is not necessarily transitive. Among other things, this affects the definition of cardinality restrictions - concepts no longer have a single cardinality in the logic.

Straccia et al. 54556869] provide 4-valued semantics for $\mathcal{A L C}$-like languages similar to but distinct from Patel-Schneider's, and in some cases sound and complete sequent calculi are provided [5569]. The interpretations for existential and universal role restrictions are the same as those used in $\mathcal{S R O I} \mathcal{Q} 4$ (from a syntactic and semantic standpoint, the $\mathcal{A L C}$ fragment of $\mathcal{S R O I} \mathcal{Q} 4$ appears to identical to the logic described in 68]), which is distinct from those of Patel-Schneider. Subsumption in the logics is what we have called weak subsumption: $C$ is subsumed by $D$ iff $p^{+}\left(C^{\mathcal{I}}\right) \subseteq$ $p^{+}\left(D^{\mathcal{I}}\right)$ in every 4-interpretation. Similarly, equivalence is the weak equivalence defined earlier. Since cardinality restrictions are not used in the logics, equality is not addressed.

The work here on $\mathcal{S R O} \mathcal{I} \mathcal{Q} 4$ and related logics constitutes an extended version of materials appearing in both 46] and 53] (neither ELP nor the empirical tests are discussed in those works, however), which are in turn based on earlier work [47484950 51]. Regarding this earlier work, the logic $\mathcal{A L C} 4$ is discussed in 47 48 49. Embedding $\mathcal{A L C} 4$ into classical $\mathcal{A L C}$ is discussed in 47] and 48, while resolution-based reasoning algorithms are presented in 48 and $49 . \mathcal{S H O I N} 4$ and its embedding into classical $\mathcal{S H O I N ~ [ 3 5 ] ~ a r e ~ p r e - ~}$ sented in 51]. In $\mathcal{S H O I N} 4$, unqualified cardinality restrictions are allowed, as are nominals (which

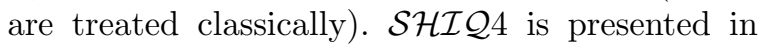
[50], as are paraconsistent versions of $\mathcal{E} \mathcal{L}^{++}$, HornDLs, and DL-Lite logics. The latter logics are analyzed more fully here and in 46]. With one exception 49, each of these papers describes embedding a paraconsistent logic into a classical counterpart. 
We are unaware of anything similar to this in the work by Patel-Schneider and Straccia et al.

Lang 45 has defined paraconsistent semantics for $\mathcal{A L C}$ and $\mathcal{S H \mathcal { Q }}$ (which, incidentally, he also calls $\mathcal{A L C} 4$ and $\mathcal{S H \mathcal { I } 4 )}$ by translating them into a paraconsistent first-order logic based on Arieli and Avron's propositional logic. As noted before, the 4-valued ELP semantics presented here is similar to his first-order logic semantics. Lang also provides separate schemes for translating his logic into FOL and for translating paraconsistent $\mathcal{A L C} 4$ and $\mathcal{S H I} \mathcal{I} 4$ directly into their classical counterparts. Paraconsistent equality and inequality are introduced as special binary relations, together with suitable axioms. An implication operator, which we refer to using $\rightarrow_{L}$, is also defined.

\begin{tabular}{c|cccc}
$\rightarrow_{L}$ & $n$ & 0 & 1 & $b$ \\
\hline$n$ & 1 & 1 & 1 & 1 \\
0 & 1 & $b$ & 1 & $b$ \\
1 & $n$ & $n$ & 1 & 1 \\
$b$ & $n$ & 0 & 1 & $b$
\end{tabular}

Since $\left\{\neg\left(\mathcal{P} \rightarrow_{L} \mathcal{Q}\right)\right\} \models_{4}(\neg \mathcal{P} \wedge \neg \mathcal{Q})$, the connective is classically unsound.

More recently, Kamide 41] describes a 4-valued paraconsistent variant of $\mathcal{A L C}$ making use of both classical and paraconsistent negation. An embedding theorem similar to the ones described here is also given, as is a sound and complete tableau algorithm. Without the additional negation, the logic appears to be the same as $\mathcal{A L C} 4$ (though there are certain notational differences). The use of the additional negation strengthens the logic considerably, however. Indeed, it appears that all classical $\mathcal{A L C}$ consequences are regained.

Zhang et al. 70 present a paraconsistent variation of $\mathcal{S H \mathcal { Q }}$ based on Besnard and Hunter's quasi-classical logic 37. It is claimed that the logic $(Q C-\mathcal{S H \mathcal { I }})$ remedies problems inherent to the 4 -valued approach described here. Specifically, a weak semantics (virtually identical to $\mathcal{S H I} \mathcal{I} 4$ ) is defined in which inclusion is based on internal inclusion. For the weak semantics, Modus Ponens, Modus Tollens, and Disjunctive Syllogism all fail. A strong semantics, building upon the weak, is provided which does not suffer from this problem (all three hold).

While it is certainly true that Disjunctive Syllogism fails for $\mathcal{S R O I} 4$ regardless of the inclusion operator used, Modus Ponens is satisfied by both internal and strong inclusion, and Modus Tollens is satisfied by strong inclusion. This is the primary point of introducing the operators in the paraconsistent logics described here (and, we imagine, in the propositional logics upon which they are based).

The failure of Disjunctive Syllogism is indeed a substantial drawback. The paraconsistent logics described here simply do not permit it under any circumstances. Arieli has recently noted 4 that Belnap's 4-valued logic is strictly weaker than quasi-classical logic ${ }^{10}$ Nevertheless, the main virtue of the paraconsistent DL framework presented here is that the logics can be embedded into classical formalisms. This in turn allows classical tools to be used to reason over even inconsistent knowledge bases. To our knowledge, no similar translation scheme has been created for $Q C$ $\mathcal{S H I} \mathcal{Q}$.

\section{Appendix}

\section{A. Proofs}

\section{A.1. Properties of Implication Operators}

Proof of Proposition 2, If $\rightsquigarrow$ satisfies Modus Ponens and the Deduction Theorem, then $\rightsquigarrow$ satisfies none of Modus Tollens, Strong Equivalence, or Transposition.

Proof. Suppose $\rightsquigarrow$ satisfies Modus Ponens and the Deduction Theorem. Let $A$ and $B$ be atomic, $\mathcal{P}=$ $(A \vee B), \mathcal{Q}=(A \wedge \neg A)$, and $K=\{\mathcal{Q}\}$. Let $v$ be a truth-value assignment such that $v(A)=b$ and $v(B)=1$. Observe that $v(\mathcal{P})=1$ and $v(\mathcal{Q})=b$, and so every element of $K$ is designated on $v$. We consider Modus Tollens, Strong Equivalence and Transposition separately.

1. Given the definitions of $\mathcal{Q}$ and $K$ above, $K \cup$ $\{\mathcal{P}\} \models_{4} \mathcal{Q}$ and $K \models_{4} \neg \mathcal{Q}$. Since $\rightsquigarrow$ satisfies the Deduction Theorem, $K \models_{4} \mathcal{P} \rightsquigarrow \mathcal{Q}$. $K$ is designated on $v$ and yet $v(\neg \mathcal{P})=0$, and so $K \not \forall_{4} \neg \mathcal{P}$. As such, $\rightsquigarrow$ does not satisfy Modus Tollens.

\footnotetext{
${ }^{10}$ The comparison was made using the simple propositional language defined over $\neg, \wedge$, and $\vee$. Arieli also points out that quasi-classical logic is in some cases too strong, in that Disjunctive Syllogism is permitted in too many cases. E.g., $\{A, \neg A, A \vee B\} \vdash_{Q C} B$, which will strike many as counter-intuitive.
} 
2. Given the definitions of $\mathcal{P}, \mathcal{Q}$, and $K$ above, it follows that $K \models{ }_{4} \mathcal{P} \rightsquigarrow \mathcal{Q}$ and $K \models_{4} \mathcal{Q} \rightsquigarrow \mathcal{P}$. $K$ is designated on $v$, and so both $(\mathcal{P} \rightsquigarrow \mathcal{Q})$ and $(\mathcal{Q} \rightsquigarrow \mathcal{P})$ are designated on $v$. Yet $v(\mathcal{P})=$ 1 and $v(\mathcal{Q})=b$, and so Strong Equivalence is not satisfied.

3. As in 1 above, $K \models_{4} \mathcal{P} \rightsquigarrow \mathcal{Q}$ and $K \models_{4} \neg \mathcal{Q}$. If $K=_{4} \neg \mathcal{Q} \rightsquigarrow \neg \mathcal{P}$, then by Modus Ponens, $K \models_{4} \neg \mathcal{P}$. However, $K$ is designated on $v$ but $v(\neg \mathcal{P})=0$, and so it can't be that $K \models{ }_{4} \neg \mathcal{Q} \rightsquigarrow \neg \mathcal{P}$. Consequently, Transposition is not satisfied.

Observe that it does not matter whether $\rightsquigarrow$ is Supraclassical or satisfies Identity. Furthermore, only in the counterexample for Transposition is Modus Ponens actually used.

\section{A.2. $\mathcal{S R O I Q 4}$}

Proof of Proposition 17, For any $\mathcal{S R O I Q} 4$ concepts $C, D$ and 4-interpretation $\mathcal{I}$, the following hold:
1. $(\neg \top)^{\mathcal{I}}=\perp^{\mathcal{I}}$
2. $(\neg \perp)^{\mathcal{I}}=\top^{\mathcal{I}}$
3. $(\neg \neg C)^{\mathcal{I}}=C^{\mathcal{I}}$
4. $(\neg(C \sqcap D))^{\mathcal{I}}=(\neg C \sqcup \neg D)^{\mathcal{I}}$
5. $(\neg(C \sqcup D))^{\mathcal{I}}=(\neg C \sqcap \neg D)^{\mathcal{I}}$
6. $(\neg \exists R . C)^{\mathcal{I}}=(\forall R . \neg C)^{\mathcal{I}}$
7. $(\neg \forall R . C)^{\mathcal{I}}=(\exists R . \neg C)^{\mathcal{I}}$
8. $(\neg \leq n R . C)^{\mathcal{I}}=(\geq n+1 R . C)^{\mathcal{I}}$
9. $(\neg \geq n R . C)^{\mathcal{I}}=(\leq n-1 R . C)^{\mathcal{I}}$

Proof. We show selected cases.

1.1. $p^{+}\left((\neg \top)^{\mathcal{I}}\right)=p^{-}\left(\top^{\mathcal{I}}\right)=\varnothing=p^{+}\left(\perp^{\mathcal{I}}\right)$

1.2. $p^{-}\left((\neg \top)^{\mathcal{I}}\right)=p^{+}\left(\top^{\mathcal{I}}\right)=\Delta^{\mathcal{I}}=p^{-}\left(\perp^{\mathcal{I}}\right)$

3.1. $p^{+}\left((\neg \neg C)^{\mathcal{I}}\right)=p^{-}\left((\neg C)^{\mathcal{I}}\right)=p^{+}\left(C^{\mathcal{I}}\right)$.

3.2. $p^{-}\left((\neg \neg C)^{\mathcal{I}}\right)=p^{+}\left((\neg C)^{\mathcal{I}}\right)=p^{-}\left(C^{\mathcal{I}}\right)$.

4.1. $p^{+}\left((\neg(C \sqcap D))^{\mathcal{I}}\right)=p^{-}\left((C \sqcap D)^{\mathcal{I}}\right)=$ $p^{-}\left(C^{\mathcal{I}}\right) \cup p^{-}\left(D^{\mathcal{I}}\right)=p^{+}\left((\neg C)^{\mathcal{I}}\right) \cup p^{+}\left((\neg D)^{\mathcal{I}}\right)$ $=p^{+}\left((\neg C \sqcup \neg D)^{\mathcal{I}}\right)$

4.2. $p^{-}\left((\neg(C \sqcap D))^{\mathcal{I}}\right)=p^{+}\left((C \sqcap D)^{\mathcal{I}}\right)=$ $p^{+}\left(C^{\mathcal{I}}\right) \cap p^{+}\left(D^{\mathcal{I}}\right)=p^{-}\left((\neg C)^{\mathcal{I}}\right) \cap p^{-}\left((\neg D)^{\mathcal{I}}\right)$ $=p^{-}\left((\neg C \sqcup \neg D)^{\mathcal{I}}\right)$

6.1. $p^{+}\left((\neg \exists R . C)^{\mathcal{I}}\right)=p^{-}\left((\exists R . C)^{\mathcal{I}}\right)=$ $\left\{x \mid(\forall y)\left[(x, y) \in p^{+}\left(R^{\mathcal{I}}\right) \mapsto y \in p^{-}\left(C^{\mathcal{I}}\right)\right]\right\}$ $=\left\{x \mid(\forall y)\left[(x, y) \in p^{+}\left(R^{\mathcal{I}}\right) \mapsto y \in p^{+}\left((\neg C)^{\mathcal{I}}\right)\right]\right\}$ $=p^{+}\left((\forall R . \neg C)^{\mathcal{I}}\right)$
6.2. $p^{-}\left((\neg \exists R . C)^{\mathcal{I}}\right)=p^{+}\left((\exists R . C)^{\mathcal{I}}\right)=$ $\left\{x \mid(\exists y)\left[(x, y) \in p^{+}\left(R^{\mathcal{I}}\right) \wedge y \in p^{+}\left(C^{\mathcal{I}}\right)\right]\right\}=$ $\left\{x \mid(\exists y)\left[(x, y) \in p^{+}\left(R^{\mathcal{I}}\right) \wedge y \in p^{-}\left((\neg C)^{\mathcal{I}}\right)\right]\right\}=$ $p^{-}\left((\forall R . \neg C)^{\mathcal{I}}\right)$
8.1. $p^{+}\left((\neg \leq n R . C)^{\mathcal{I}}\right)=p^{-}\left((\leq n R . C)^{\mathcal{I}}\right)=$ $\left\{x \mid \sharp\left\{y \mid(x, y) \in p^{+}\left(R^{\mathcal{I}}\right) \wedge y \in p^{+}\left(C^{\mathcal{I}}\right)\right\}>n\right\}$ $=\left\{x \mid \sharp\left\{y \mid(x, y) \in p^{+}\left(R^{\mathcal{I}}\right) \wedge y \in p^{+}\left(C^{\mathcal{I}}\right)\right\} \geq\right.$ $(n+1)\}=p^{+}\left((\geq(n+1) R . C)^{\mathcal{I}}\right)$
8.2. $p^{-}\left((\neg \leq n R . C)^{\mathcal{I}}\right)=p^{+}\left((\leq n R . C)^{\mathcal{I}}\right)=$ $\left\{x \mid \sharp\left\{y \mid(x, y) \in p^{+}\left(R^{\mathcal{I}}\right) \wedge y \notin p^{-}\left(C^{\mathcal{I}}\right)\right\} \leq n\right\}$ $=\left\{x \mid \sharp\left\{y \mid(x, y) \in p^{+}\left(R^{\mathcal{I}}\right) \wedge y \notin p^{-}\left(C^{\mathcal{I}}\right)\right\}<\right.$ $(n+1)\}=p^{-}\left((\geq(n+1) R . C)^{\mathcal{I}}\right)$

Proof of Proposition 20, Let $C$ and $D$ be $\mathcal{S R O I Q 4}$ concepts.

1. $C$ is weakly subsumed by $D$ iff $C \sqsubset D$ is satisfied on each $\mathcal{I}$.

2. $C$ is strongly subsumed by $D$ iff $C \rightarrow D$ is satisfied on each $\mathcal{I}$.

3. $C$ and $D$ are weakly equivalent iff $C \sqsubset D$ and $D \sqsubset C$ are both satisfied on each $\mathcal{I}$.

4. $C$ and $D$ are strongly equivalent iff $C \rightarrow D$ and $D \rightarrow C$ are both satisfied on each $\mathcal{I}$.

Proof.

1. This follows directly from the definition of weak subsumption and satisfaction $C \sqsubset D$.

2. Again, this follows by definition of strong subsumption and satisfaction $C \rightarrow D$.

3. If $(C \leftrightarrow D)$, then for any $\mathcal{I}, C^{\mathcal{I}}=D^{\mathcal{I}}$, and so $p^{+}\left(C^{\mathcal{I}}\right) \subseteq p^{+}\left(D^{\mathcal{I}}\right)$ and $p^{-}\left(D^{\mathcal{I}}\right) \subseteq p^{-}\left(C^{\mathcal{I}}\right)$, and $p^{+}\left(D^{\mathcal{I}}\right) \subseteq p^{+}\left(C^{\mathcal{I}}\right)$ and $p^{-}\left(C^{\mathcal{I}}\right) \subseteq$ $p^{-}\left(D^{\mathcal{I}}\right)$. Both $(C \rightarrow D)$ and $(D \rightarrow C)$ are satisfied by $\mathcal{I}$. If $(C \rightarrow D)$ and $(D \rightarrow$ $C)$ are satisfied by $\mathcal{I}$, then by definition, $p^{+}\left(C^{\mathcal{I}}\right) \subseteq p^{+}\left(D^{\mathcal{I}}\right)$ and $p^{-}\left(D^{\mathcal{I}}\right) \subseteq p^{-}\left(C^{\mathcal{I}}\right)$, and $p^{+}\left(D^{\mathcal{I}}\right) \subseteq p^{+}\left(C^{\mathcal{I}}\right)$ and $p^{-}\left(C^{\mathcal{I}}\right) \subseteq$ $p^{-}\left(D^{\mathcal{I}}\right)$. Hence, $C^{\mathcal{I}}=D^{\mathcal{I}}$. Generalizing on $\mathcal{I}$, $(C \leftrightarrow D)$.

4. Clearly, $p^{+}\left(C^{\mathcal{I}}\right)=p^{+}\left(D^{\mathcal{I}}\right)$ iff $p^{+}\left(C^{\mathcal{I}}\right) \subseteq$ $p^{+}\left(D^{\mathcal{I}}\right)$ and $p^{+}\left(D^{\mathcal{I}}\right) \subseteq p^{+}\left(C^{\mathcal{I}}\right)$.

Below, $\Delta$ refers to the domain of discourse shared by both $\mathcal{I}_{4}$ and $\mathcal{I}_{2}$.

Proof of Proposition 21. If $\mathcal{I}_{2}$ is a 2-interpretation and $\mathcal{I}_{4}$ its 4 -counterpart, then for any concept $C$

$$
C^{\mathcal{I}_{4}}=\left\langle C^{\mathcal{I}_{2}}, \Delta-C^{\mathcal{I}_{2}}\right\rangle .
$$


Proof. We induct on the degree of $C$. If $C \in N_{C} \cup$ $N_{o}$ or $C=\exists R$.Self $f$ the claim holds by definition. For $\top, p^{+}\left(\top^{\mathcal{I}_{4}}\right)=\Delta=\top^{\mathcal{I}_{2}}$ and $p^{-}\left(\top^{\mathcal{I}_{4}}\right)=\varnothing=$ $\Delta-\Delta=\Delta-\top^{\mathcal{I}_{2}}$. For bottom, $p^{+}\left(\perp^{\mathcal{I}_{4}}\right)=\varnothing=$ $\perp^{\mathcal{I}_{2}}$ and $p^{-}\left(\perp^{\mathcal{I}_{4}}\right)=\Delta=\Delta-\varnothing=\Delta-\perp^{\mathcal{I}_{2}}$. For the inductive cases, suppose the claim holds for all concepts of degree $<n$ and that $C$ has degree $n$. We consider the cases where $C$ is one of $\neg D$, $(D \sqcap E),(\exists R . D)$, or $(\geq n R . D)$. The remaining cases are similar.

$$
\begin{aligned}
& -(\neg \mathbf{D})^{\mathcal{I}_{4}}=\left\langle p^{-}\left(D^{\mathcal{I}_{4}}\right), p^{+}\left(D^{\mathcal{I}_{4}}\right)\right\rangle= \\
& \left.\left.\left\langle\Delta-D^{\mathcal{I}_{2}}, D^{\mathcal{I}_{2}}\right)\right\rangle=\left\langle(\neg D)^{\mathcal{I}_{2}}, \Delta-(\neg D)^{\mathcal{I}_{2}}\right)\right\rangle \text {. } \\
& -(\mathbf{D} \sqcap \mathbf{E})^{\mathcal{I}_{4}}= \\
& \left\langle\left(p^{+}\left(D^{\mathcal{I}_{4}}\right) \cap p^{+}\left(E^{\mathcal{I}_{4}}\right)\right),\left(p^{-}\left(D^{\mathcal{I}_{4}}\right) \cup p^{-}\left(E^{\mathcal{I}_{4}}\right)\right)\right\rangle \\
& =\left\langle\left(D^{\mathcal{I}_{2}} \cap E^{\mathcal{I}_{2}}\right),\left(\left(\Delta-D^{\mathcal{I}_{2}}\right) \cup\left(\Delta-E^{\mathcal{I}_{2}}\right)\right)\right\rangle \\
& =\left\langle\left(D^{\mathcal{I}_{2}} \cap E^{\mathcal{I}_{2}}\right),\left(\Delta-\left(D^{\mathcal{I}_{2}} \cap E^{\mathcal{I}_{2}}\right)\right)\right\rangle \text {. } \\
& -(\exists \mathbf{R} . \mathbf{D})^{\mathcal{I}_{4}}=\langle P, N\rangle \text {, where } \\
& P=\left\{x \mid(\exists y)\left[(x, y) \in p^{+}\left(R^{\mathcal{I}_{4}}\right) \wedge y \in p^{+}\left(D^{\mathcal{I}_{4}}\right)\right]\right\} \\
& =\left\{x \mid(\exists y)\left[(x, y) \in R^{\mathcal{I}_{2}} \wedge y \in D^{\mathcal{I}_{2}}\right]\right\}= \\
& (\exists R . D)^{\mathcal{I}_{2}} ; \text { and } N= \\
& \left\{x \mid(\forall y)\left[(x, y) \in p^{+}\left(R^{\mathcal{I}_{4}}\right) \mapsto y \in p^{-}\left(D^{\mathcal{I}_{4}}\right)\right]\right\} \\
& =\left\{x \mid(\forall y)\left[(x, y) \in R^{\mathcal{I}_{2}} \mapsto y \in\left(\Delta-D^{\mathcal{I}_{2}}\right)\right]\right\} \\
& =\left\{x \mid(\forall y)\left[(x, y) \in R^{\mathcal{I}_{2}} \mapsto y \notin D^{\mathcal{I}_{2}}\right]\right\}= \\
& \left\{x \mid \neg \neg(\forall y)\left[(x, y) \in R^{\mathcal{I}_{2}} \mapsto y \notin D^{\mathcal{I}_{2}}\right]\right\}= \\
& \left\{x \mid \neg(\exists y)\left[(x, y) \in R^{\mathcal{I}_{2}} \wedge y \in D^{\mathcal{I}_{2}}\right]\right\}=\Delta- \\
& (\exists R . D)^{\mathcal{I}_{2}} \\
& \text { - }(\geq \mathbf{n R} . \mathbf{D})^{\mathcal{I}_{4}}=\langle P, N\rangle \text {, where } P= \\
& \left\{x \mid \sharp\left\{y \mid(x, y) \in p^{+}\left(R^{\mathcal{I}_{4}}\right) \wedge y \in p^{+}\left(D^{\mathcal{I}_{4}}\right)\right\} \geq n\right\} \\
& =\left\{x \mid \sharp\left\{y \mid(x, y) \in R^{\mathcal{I}_{2}} \wedge y \in D^{\mathcal{I}_{2}}\right\} \geq n\right\}= \\
& D^{\mathcal{I}_{2}} ; \text { and } N= \\
& \left\{x \mid \sharp\left\{y \mid(x, y) \in p^{+}\left(R^{\mathcal{I}_{4}}\right) \wedge y \notin p^{-}\left(D^{\mathcal{I}_{4}}\right)\right\}<n\right\} \\
& =\left\{x \mid \sharp\left\{y \mid(x, y) \in p^{+}\left(R^{\mathcal{I}_{4}}\right) \wedge y \in p^{+}\left(D^{\mathcal{I}_{4}}\right)\right\}<\right. \\
& n\}=\left\{x \mid \sharp\left\{y \mid(x, y) \in R^{\mathcal{I}_{2}} \wedge y \in D^{\mathcal{I}_{2}}\right\}<n\right\} \\
& =\Delta-(\geq n R \cdot D)^{\mathcal{I}_{2}} \text {. }
\end{aligned}
$$

The below two lemmas are needed to prove Proposition 22, which relates the 2-models of an axiom $A$ to its 4 -models.

Lemma 61. Let $\mathcal{I}_{2}$ be a 2-interpretation and $\mathcal{I}_{4}$ its 4-counterpart. If $R \in N_{R}$, then $R^{-\mathcal{I}_{4}}=$ $\left\langle R^{-\mathcal{I}_{2}}, \Delta^{2}-R^{-\mathcal{I}_{2}}\right\rangle$.

Proof. By definition of $\mathcal{I}_{4}, R^{\mathcal{I}_{4}}=\left\langle R^{\mathcal{I}_{2}}, \Delta^{2}-R^{\mathcal{I}_{2}}\right\rangle$. From this, $R^{-\mathcal{I}_{4}}=\left\langle R^{-\mathcal{I}_{2}},\left(\Delta^{2}-R^{\mathcal{I}_{2}}\right)^{-}\right\rangle$. Observe that $\left(\Delta^{2}-R^{\mathcal{I}_{2}}\right)^{-}=\left(\Delta^{2}\right)^{-}{ }^{-}\left(R^{\mathcal{I}_{2}}\right)^{-}=\Delta^{2}-R^{-\mathcal{I}_{2}}$. And so $\left.R^{-\mathcal{I}_{4}}=\left\langle R^{-\mathcal{I}_{2}}, \Delta^{2}-R^{-\mathcal{I}_{2}}\right)\right\rangle$.

Lemma 62. If $\mathcal{I}_{2}$ is a 2-interpretation and $\mathcal{I}_{4}$ its 4-counterpart, then for roles $R_{1}, \ldots, R_{n},\left(R_{1} \circ \ldots \circ\right.$ $\left.R_{n}\right)^{\mathcal{I}_{4}}=\left\langle\left(R_{1} \circ \ldots \circ R_{n}\right)^{\mathcal{I}_{2}}, \Delta^{2}-\left(R_{1} \circ \ldots \circ R_{n}\right)^{\mathcal{I}_{2}}\right\rangle$.
Proof. That $p^{+}\left(\left(R_{1} \circ \ldots \circ R_{n}\right)^{\mathcal{I}_{4}}\right)=\left(R_{1} \circ \ldots \circ\right.$ $\left.R_{n}\right)^{\mathcal{I}_{2}}$ and $p^{-}\left(\left(R_{1} \circ \ldots \circ R_{n}\right)^{\mathcal{I}_{4}}\right)=\Delta^{2}-\left(R_{1} \circ \ldots \circ\right.$ $\left.R_{n}\right)^{\mathcal{I}_{2}}$ follows by definition of $\mathcal{I}_{4}$ for roles and Lemma 61.

Proof of Proposition 22, If $\mathcal{I}_{2}$ is a 2-interpretation and $\mathcal{I}_{4}$ its 4 -counterpart, then for any axiom $A, \mathcal{I}_{4}$ is a 4 -model of $A$ iff $\mathcal{I}_{2}$ is a 2-model of $A$.

Proof. (By cases):

- $\mathbf{A}=\mathbf{C}(\mathbf{a}): \mathcal{I}_{4}$ is a 4 -model of $C(a)$ iff $a^{\mathcal{I}_{4}} \in$ $p^{+}\left(C^{\mathcal{I}_{4}}\right)$ iff $a^{\mathcal{I}_{2}} \in C^{\mathcal{I}_{2}}$ iff $\mathcal{I}_{2}$ is a 2-model of $C(a)$.

- $\mathbf{A}=\mathbf{R}(\mathbf{a}, \mathbf{b}): \mathcal{I}_{4}$ is a 4 -model of $R(a, b)$ iff $\left(a^{\mathcal{I}_{4}}, b^{\mathcal{I}_{4}}\right) \in p^{+}\left(R^{\mathcal{I}_{4}}\right)$ iff $\left(a^{\mathcal{I}_{2}}, b^{\mathcal{I}_{2}}\right) \in R^{\mathcal{I}_{2}}$ iff $\mathcal{I}_{2}$ is a 2-model of $R(a, b)$.

- $\mathbf{A}=\neg \mathbf{R}(\mathbf{a}, \mathbf{b}): \mathcal{I}_{4}$ is a 4 -model of $\neg R(a, b)$ iff $\left(a^{\mathcal{I}_{4}}, b^{\mathcal{I}_{4}}\right) \in p^{-}\left(R^{\mathcal{I}_{4}}\right)$ iff $\left(a^{\mathcal{I}_{2}}, b^{\mathcal{I}_{2}}\right) \notin R^{\mathcal{I}_{2}}$ iff $\mathcal{I}_{2}$ is a 2 -model of $\neg R(a, b)$.

- $\mathbf{A}=\mathbf{C} \sqsubset \mathbf{D}: \mathcal{I}_{4}$ is a 4-model of $(C \sqsubset D)$ iff $p^{+}\left(C^{\mathcal{I}_{4}}\right) \subseteq p^{+}\left(D^{\mathcal{I}_{4}}\right)$ iff $C^{\mathcal{I}_{2}} \subseteq D^{\mathcal{I}_{2}}$ iff $\mathcal{I}_{2}$ is a 2-model of $C \sqsubset D$.

- $\mathbf{A}=\mathbf{C} \mapsto \mathbf{D}: \mathcal{I}_{4}$ is a 4-model of $(C \mapsto D)$ iff $\Delta-p^{-}\left(C^{\mathcal{I}_{4}}\right) \subseteq p^{+}\left(D^{\mathcal{I}_{4}}\right)$ iff $p^{+}\left(C^{\mathcal{I}_{4}}\right) \subseteq$ $p^{+}\left(D^{\mathcal{I}_{4}}\right)$ iff $C^{\mathcal{I}_{2}} \subseteq D^{\mathcal{I}_{2}}$ iff $\mathcal{I}_{2}$ is a 2-model of $C \mapsto D$.

- $\mathbf{A}=\mathbf{C} \rightarrow \mathbf{D}: \mathcal{I}_{4}$ is a 4 -model of $(C \rightarrow D)$ iff $\left(p^{+}\left(C^{\mathcal{I}_{4}}\right) \subseteq p^{+}\left(D^{\mathcal{I}_{4}}\right)\right.$ and $p^{-}\left(D^{\mathcal{I}_{4}}\right) \subseteq$ $\left.p^{-}\left(C^{\mathcal{I}_{4}}\right)\right)$ iff $\left(C^{\mathcal{I}_{2}} \subseteq D^{\mathcal{I}_{2}}\right.$ and $\Delta-p^{-}\left(C^{\mathcal{I}_{4}}\right) \subseteq$ $\left.\Delta-p^{-}\left(D^{\mathcal{I}_{4}}\right)\right)$ iff $\left(C^{\mathcal{I}_{2}} \subseteq D^{\mathcal{I}_{2}}\right.$ and $\left.C^{\mathcal{I}_{2}} \subseteq D^{\mathcal{I}_{2}}\right)$ iff $\mathcal{I}_{2}$ is a 2 -model of $C \rightarrow D$.

- $\mathbf{A}=\mathbf{R}_{\mathbf{1}} \circ \ldots \circ \mathbf{R}_{\mathbf{n}} \sqsubset \mathbf{R}_{\mathbf{n}+\mathbf{1}}: \mathcal{I}_{4}$ is a 4-model of $R_{1} \circ \ldots \circ R_{n} \sqsubset R_{n+1}$ iff $p^{+}\left(\left(R_{1} \circ \ldots \circ R_{n}\right)^{\mathcal{I}_{4}}\right)$ $\subseteq p^{+}\left(R_{n+1}^{\mathcal{I}_{4}}\right)$ iff $\left(R_{1} \circ \ldots \circ R_{n}\right)^{\mathcal{I}_{2}} \subseteq R_{n+1}^{\mathcal{I}_{2}}$ iff $\mathcal{I}_{2}$ is a 2-model of $R_{1} \circ \ldots \circ R_{n} \sqsubset R_{n+1}$.

- $\mathbf{A}=\boldsymbol{\operatorname { R e f }}(\mathbf{R}): \mathcal{I}_{4}$ is a 4 -model of $\operatorname{Ref}(R)$ iff $\{(x, x) \mid x \in \Delta\} \subseteq p^{+}\left(\left(R^{\mathcal{I}_{4}}\right)\right)$ iff $\{(x, x) \mid x \in$ $\Delta\} \subseteq R^{\mathcal{I}_{2}}$ iff $\mathcal{I}_{2}$ is a 2-model of $\operatorname{Ref}(R)$.

- $\mathbf{A}=\operatorname{Irr}(\mathbf{R}): \mathcal{I}_{4}$ is a 4-model of $\operatorname{Irr}(R)$ iff $\{(x, x) \mid x \in \Delta\} \subseteq p^{-}\left(\left(R^{\mathcal{I}_{4}}\right)\right)$ iff $\{(x, x) \mid x \in$ $\Delta\} \subseteq(\Delta \times \Delta)-R^{\mathcal{I}_{2}}$ iff $\{(x, x) \mid x \in \Delta\} \cap R^{\mathcal{I}_{2}}=$ $\varnothing$ iff $\mathcal{I}_{2}$ is a 2 -model of $\operatorname{Irr}(R)$.

- $\mathbf{A}=\operatorname{Dis}(\mathbf{R}, \mathbf{S}): \mathcal{I}_{4}$ is a 4 -model of $\operatorname{Dis}(R, S)$ iff $\left(p^{+}\left(R^{\mathcal{I}_{4}}\right) \subseteq p^{-}\left(S^{\mathcal{I}_{4}}\right)\right.$ and $p^{+}\left(S^{\mathcal{I}_{4}}\right) \subseteq$ $\left.p^{-}\left(R^{\mathcal{I}_{4}}\right)\right)$ iff $\left(R^{\mathcal{I}_{2}} \subseteq \Delta^{2}-S^{\mathcal{I}_{2}}\right.$ and $S^{\mathcal{I}_{2}} \subseteq$ $\left.\Delta^{2}-R^{\mathcal{I}_{2}}\right)$ iff $R^{\mathcal{I}_{2}} \cap S^{\mathcal{I}_{2}}=\varnothing$ iff $\mathcal{I}_{2}$ is a 2-model of $\operatorname{Dis}(R, S)$.

Since (in)equality assertions have the same semantics in both 2-interpretations and 4-interpretations, we need show nothing for them. 


\section{A.3. Removing Gaps and Gluts}

Proof of Proposition 30. A 4-valued interpretation $\mathcal{I}$ of $E M(K B)$ is a 4-model of $E M(K B)$ iff for each concept $C$ of $K B, p^{+}\left(C^{\mathcal{I}}\right) \cup p^{-}\left(C^{\mathcal{I}}\right)=\Delta^{\mathcal{I}}$.

Proof. (LR) Let $\mathcal{I}$ 4-model $E M(K B)$. We induct on the degree of the concept $C$. For $C=\top$ and $C=\perp$, the claim holds by definition of $\top$ and $\perp$. If $C \in N_{C} \cup N_{o}$ or has the form $\exists$ R.Self, since $\mathcal{I}$ 4-satisfies $E M(K B), \Delta^{\mathcal{I}} \subseteq p^{+}\left(C^{\mathcal{I}}\right) \cup p^{+}\left((\neg C)^{\mathcal{I}}\right)$. Since $p^{+}\left(C^{\mathcal{I}}\right) \cup p^{+}\left((\neg C)^{\mathcal{I}}\right) \subseteq \Delta^{\mathcal{I}}$, the claim must hold. For the inductive cases, suppose the claim holds for concepts of degree $<n$ and that $C$ has degree $n$. We consider selective cases. Proofs for the cases left out are analogous.

1. $\mathbf{C}=(\mathbf{D} \sqcap \mathbf{E})$ : If $d \notin p^{+}\left(C^{\mathcal{I}}\right)$, then $d \notin$ $p^{+}\left(D^{\mathcal{I}}\right)$ or $d \notin p^{+}\left(E^{\mathcal{I}}\right)$. By ind. hyp., $d \in$ $p^{-}\left(D^{\mathcal{I}}\right)$ or $d \in p^{-}\left(E^{\mathcal{I}}\right)$, and so $d \in p^{-}((D \sqcap$ $\left.E)^{\mathcal{I}}\right)$. I.e., $d \in p^{-}\left(C^{\mathcal{I}}\right)$.

2. $\mathbf{C}=(\forall \mathbf{R} . \mathbf{D})$ : If $d \notin p^{+}\left(C^{\mathcal{I}}\right)$, then there exists a $d^{\prime}$ such that $\left(d, d^{\prime}\right) \in p^{+}\left(R^{\mathcal{I}}\right)$ and $d^{\prime} \notin$ $p^{+}\left(D^{\mathcal{I}}\right)$. But then by ind. hyp., $d^{\prime} \in p^{-}\left(D^{\mathcal{I}}\right)$, and so $d \in p^{-}\left(C^{\mathcal{I}}\right)$.

3. $\mathbf{C}=(\leq \mathbf{n R} . \mathbf{D})$ : If $d \notin p^{+}\left(C^{\mathcal{I}}\right)$, then

$\left.\sharp\left\{y \mid(d, y) \in p^{+}\left(R^{\mathcal{I}}\right) \wedge y \notin p^{-}\left(D^{\mathcal{I}}\right)\right\}>n\right\}$. But then by ind. hyp., $\sharp\left\{y \mid(d, y) \in p^{+}\left(R^{\mathcal{I}}\right) \wedge y \in\right.$ $\left.\left.p^{+}\left(D^{\mathcal{I}}\right)\right\}>n\right\}$, and so $d \in p^{-}\left(C^{\mathcal{I}}\right)$.

(RL) Suppose for each concept $C, p^{+}\left(C^{\mathcal{I}}\right) \cup$ $p^{-}\left(C^{\mathcal{I}}\right)=\Delta^{\mathcal{I}}$. Then $p^{+}\left(C^{\mathcal{I}}\right) \cup p^{+}\left((\neg C)^{\mathcal{I}}\right)=\Delta^{\mathcal{I}}$, $\Delta \subseteq p^{+}\left(C^{\mathcal{I}}\right) \cup p^{+}\left((\neg C)^{\mathcal{I}}\right)$, and so $p^{+}\left(\top^{\mathcal{I}}\right) \subseteq$ $p^{+}\left((C \sqcup \neg C)^{\mathcal{I}}\right) . \mathcal{I}$ is a 4 -model of $\top \sqsubset(C \sqcup \neg C)$. Generalizing on $C, \mathcal{I}$ is a 4 -model of $\operatorname{EM}(K B)$.

Proof of Proposition 31. A 4-valued interpretation $\mathcal{I}$ of $E F Q(K B)$ is a 4-model of $E F Q(K B)$ iff for each concept $C$ of $K B, p^{+}\left(C^{\mathcal{I}}\right) \cap p^{-}\left(C^{\mathcal{I}}\right)=\varnothing$.

Proof. (LR) Suppose $\mathcal{I} 4$-satisfies $E F Q(K B)$. We induct on the degree of $C$. For $C=\top$ and $C=\perp$, the claim holds by definition. If $C \in N_{C} \cup N_{o}$ or has the form $\exists$ R.Self,$(C \sqcap \neg C) \sqsubset \perp$ is satisfied, and so $\left.p^{+}\left(C^{\mathcal{I}}\right) \cap p^{+}(\neg C)^{\mathcal{I}}\right) \subseteq \varnothing$. However, $\varnothing \subseteq$ $\left.p^{+}\left(C^{\mathcal{I}}\right) \cap p^{+}(\neg C)^{\mathcal{I}}\right)$, and so $p^{+}\left(C^{\mathcal{I}}\right) \cap p^{-}\left(C^{\mathcal{I}}\right)=\varnothing$. For the inductive cases, suppose the claim holds for concepts of degree $<n$ and that $C$ has degree $n$. We again consider selected cases.

1. If $d \in p^{+}\left((\mathbf{D} \sqcup \mathbf{E})^{\mathcal{I}}\right)$, then $d \in p^{+}\left(D^{\mathcal{I}}\right)$ or $d \in p^{+}\left(E^{\mathcal{I}}\right)$. By ind. hyp., $d \notin p^{-}\left(D^{\mathcal{I}}\right)$ or $d \notin p^{-}\left(E^{\mathcal{I}}\right)$, and so $d \notin p^{-}\left((D \sqcup E)^{\mathcal{I}}\right)$.
2. If $d \in p^{+}\left((\exists \mathbf{R} . \mathbf{D})^{\mathcal{I}}\right)$, then there is a $d^{\prime}$ such that $\left(d, d^{\prime}\right) \in p^{+}\left(R^{\mathcal{I}}\right)$ and $d^{\prime} \in p^{+}\left(D^{\mathcal{I}}\right)$. However, by ind. hyp., $d^{\prime} \notin p^{-}\left(D^{\mathcal{I}}\right)$. And so $d \notin p^{-}\left((\exists R . D)^{\mathcal{I}}\right)$.

3. If $d \in p^{-}\left((\leq \mathbf{n R} . \mathbf{D})^{\mathcal{I}}\right)$, then $\sharp\{y \mid(d, y) \in$ $\left.p^{+}\left(R^{\mathcal{I}}\right) \wedge y \in p^{+}\left(D^{\mathcal{I}}\right)\right\}>n$. By ind. hyp., $\sharp\left\{y \mid(d, y) \in p^{+}\left(R^{\mathcal{I}}\right) \wedge y \notin p^{-}\left(D^{\mathcal{I}}\right)\right\}>n$, and so $d \notin p^{+}\left((\leq n R . D)^{\mathcal{I}}\right)$.

(RL) If $p^{+}\left(C^{\mathcal{I}}\right) \cap p^{-}\left(C^{\mathcal{I}}\right)=\varnothing$, then $p^{+}\left(C^{\mathcal{I}}\right) \cap$ $p^{+}\left((\neg C)^{\mathcal{I}}\right)=\varnothing, p^{+}\left((C \sqcap \neg C)^{\mathcal{I}}\right)=\varnothing$, and so $(C \sqcap \neg C) \sqsubseteq \perp$ is satisfied by $\mathcal{I}$. Generalizing, $\mathcal{I}$ 4-satisfies $\bar{E} F Q(K B)$.

Proof of Proposition $32, \mathcal{I}_{4} 4$-satisfies

$$
E M(K B) \cup E F Q(K B)
$$

iff for each concept $C, C^{\mathcal{I}_{4}}=\left\langle C^{\mathcal{I}_{2}}, \Delta-C^{\mathcal{I}_{2}}\right\rangle$.

Proof. (LR) Suppose $\mathcal{I}_{4}$ 4-satisfies $E M(K B) \cup$ $E F Q(K B)$. Let $C$ be a concept of $K B$. In virtue of Props. 30 and 31. $\Delta-p^{+}\left(C^{\mathcal{I}_{4}}\right)=p^{-}\left(C^{\mathcal{I}_{4}}\right)$. Inducting on the degree of $C$, we show that $C^{\mathcal{I}_{4}}=$ $\left\langle C^{\mathcal{I}_{2}}, \Delta-C^{\mathcal{I}_{2}}\right\rangle$. We consider two cases below, both requiring that $\Delta-p^{+}\left(C^{\mathcal{I}_{4}}\right)=p^{-}\left(C^{\mathcal{I}_{4}}\right)$. Other cases are similar.

1. $p^{+}\left((\neg \mathbf{C})^{\mathcal{I}_{4}}\right)=p^{-}\left(C^{\mathcal{I}_{4}}\right)=\Delta-C^{\mathcal{I}_{2}}=(\neg C)^{\mathcal{I}_{2}}$. So, $\Delta-(\neg C)^{\mathcal{I}_{2}}=p^{-}\left((\neg C)^{\mathcal{I}_{4}}\right)$.

2. $p^{+}\left((\leq \mathbf{n R} . \mathbf{C})^{\mathcal{I}_{4}}\right)=\left\{x \mid \sharp\left\{y \mid(x, y) \in p^{+}\left(R^{\mathcal{I}_{4}}\right) \wedge\right.\right.$ $\left.\left.y \notin p^{-}\left(C^{\mathcal{I}_{4}}\right)\right\} \leq n\right\}=$

$\left\{x \mid \sharp\left\{y \mid(x, y) \in R^{\mathcal{I}_{2}} \wedge y \in p^{+}\left(C^{\mathcal{I}_{4}}\right)\right\} \leq n\right\}$ $=\left\{x \mid \sharp\left\{y \mid(x, y) \in R^{\mathcal{I}_{2}} \wedge y \in C^{\mathcal{I}_{2}}\right\} \leq \bar{n}\right\}=$ $(\leq n R . C)^{\mathcal{I}_{2}}$. And so $\Delta-(\leq n R . C)^{\mathcal{I}_{2}}=p^{-}((\leq$ $\left.n R . C)^{\mathcal{I}_{4}}\right)$.

(RL) If $C^{\mathcal{I}_{4}}=\left\langle C^{\mathcal{I}_{2}}, \Delta-C^{\mathcal{I}_{2}}\right\rangle$, then

$\Delta \subseteq\left(p^{+}\left(C^{\mathcal{I}_{4}}\right) \cup p^{+}\left(\neg C^{\mathcal{I}_{4}}\right)\right)$ and $\left(p^{+}\left(C^{\mathcal{I}_{4}}\right) \cap\right.$ $\left.p^{+}\left((\neg C)^{\mathcal{I}_{4}}\right)\right) \subseteq \varnothing$. As such, $\mathcal{I}_{4}$ 4-satisfies $\top \sqsubset$ $(C \sqcup \neg C)$ and $(C \sqcap \neg C) \sqsubset \perp$.

Proof of Proposition 33 . Let $\mathcal{I}_{4}$ be a 4 -model of $\operatorname{EM}(K B) \cup E F Q(K B)$. If $A$ is a $\mathcal{S R O} \mathcal{I} \mathcal{Q} 4$ axiom of $K B$ and not of the form $\neg R(a, b), \operatorname{Irr}(R)$, or $\operatorname{Dis}(R, S)$, then $\mathcal{I}_{2}$ is a 2 -model of $A$ iff $\mathcal{I}_{4}$ is a 4-model of $A$.

Proof. We treat each case:

- $\mathcal{I}_{4}$ 4-satisfies $(\mathbf{C} \sqsubset \mathbf{D})$ (or $\left.\mathbf{C} \sqsubseteq \mathbf{D}\right)$ iff $p^{+}\left(C^{\mathcal{I}_{4}}\right) \subseteq p^{+}\left(D^{\mathcal{I}_{4}}\right)$ iff $C^{\mathcal{I}_{2}} \subseteq D^{\mathcal{I}_{2}}$.

- $\mathcal{I}_{4}$ 4-satisfies $(\mathbf{C} \mapsto \mathbf{D})$ iff $\Delta-p^{-}\left(C^{\mathcal{I}_{4}}\right) \subseteq$ $p^{+}\left(D^{\mathcal{I}_{4}}\right)$ iff $p^{+}\left(C^{\mathcal{I}_{4}}\right) \subseteq p^{+}\left(D^{\mathcal{I}_{4}}\right)$ iff $C^{\mathcal{I}_{2}} \subseteq$ $D^{\mathcal{I}_{2}}$. 
- $\mathcal{I}_{4}$ 4-satisfies $(\mathbf{C} \rightarrow \mathbf{D})$ iff $\left(p^{+}\left(C^{\mathcal{I}_{4}}\right) \subseteq\right.$ $p^{+}\left(D^{\mathcal{I}_{4}}\right)$ and $\left.p^{-}\left(D^{\mathcal{I}_{4}}\right) \subseteq p^{-}\left(C^{\mathcal{I}_{4}}\right)\right)$ iff $\left(C^{\mathcal{I}_{2}} \subseteq\right.$ $D^{\mathcal{I}_{2}}$ and $\left.\Delta-D^{\mathcal{I}_{2}} \subseteq \Delta-C^{\mathcal{I}_{2}}\right)$ iff $C^{\mathcal{I}_{2}} \subseteq D^{\mathcal{I}_{2}^{-}}$.

- $\mathcal{I}_{4}$ 4-satisfies $\mathbf{R}_{\mathbf{1}} \circ \ldots \circ \mathbf{R}_{\mathbf{n}} \sqsubset \mathbf{R}_{\mathbf{n}+\mathbf{1}}$ iff $p^{+}\left(\left(R_{1} \circ \ldots \circ R_{n}\right)^{\mathcal{I}_{4}}\right) \subseteq p^{+}\left(R_{n+1}^{\mathcal{I}_{4}}\right)$ iff $\left(R_{1} \circ\right.$ $\left.\ldots \circ R_{n}\right)^{\mathcal{I}_{2}} \subseteq R_{n+1}^{\mathcal{I}_{2}}$.

- $\mathcal{I}_{4}$ 4-satisfies $\boldsymbol{\operatorname { R e f }}(\mathbf{R})$ iff $\{(x, x) \mid x \in \Delta\} \subseteq$ $p^{+}\left(R^{\mathcal{I}_{4}}\right)$ iff $\{(x, x) \mid x \in \Delta\} \subseteq R^{\mathcal{I}_{2}}$.

- $\mathcal{I}_{4} 4$-satisfies $\mathbf{C}(\mathbf{a})$ iff $a^{\mathcal{I}_{4}} \in p^{+}\left(C^{\mathcal{I}_{4}}\right)$ iff $a^{\mathcal{I}_{2}} \in$ $C^{\mathcal{I}_{2}}$

- $\mathcal{I}_{4}$ 4-satisfies $\mathbf{R}(\mathbf{a}, \mathbf{b})$ iff $\left(a^{\mathcal{I}_{4}}, b^{\mathcal{I}_{4}}\right) \in p^{+}\left(R^{\mathcal{I}_{4}}\right)$ iff $\left(a^{\mathcal{I}_{2}}, b^{\mathcal{I}_{2}}\right) \in R^{\mathcal{I}_{2}}$

Since (in)equalities are treated the same in the logics, we need not consider them.

Proof of Proposition 34. If $K B$ is a $\mathcal{S R O I Q 4}$ knowledge base lacking axioms of the form $\operatorname{Irr}(R)$, $\operatorname{Dis}(R, S)$, or $\neg R(a, b)$ then $K B \cup E M(K B) \cup$ $E F Q(K B)$ has a 4-model iff $K B$ has a 2-model.

Proof. (LR) Let $\mathcal{I}_{4}$ 4-model $K B \cup E M(K B) \cup$ $\operatorname{EFQ}(K B)$. Prop. 33 applies, and so $\mathcal{I}_{2}$ (as described above) 2 -satisfies $K B$. (RL) Let $\mathcal{I}_{2}$ be any 2 -model of $K B$. We may define a 4 -counterpart $\mathcal{I}_{4}$ as in Section 3 . By Proposition 22, for any

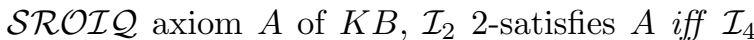
4-satisfies $A$. And so $\mathcal{I}_{4}$ 4-satisfies $K B$. Clearly, in any 2-interpretation, every axiom of $\operatorname{EM}(K B)$ and $E F Q(K B)$ is satisfied. Again by Proposition $22 . \mathcal{I}_{4}$ 4-satisfies $E M(K B), E F Q(K B)$.

\section{A.4. From $\mathcal{S R O I Q 4}$ to $\mathcal{S R O I Q}$}

Proposition 37. For any 4-interpretation $\mathcal{I}$, primed

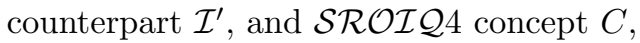

1. $p^{+}\left((C)^{\mathcal{I}}\right)=\pi(C)^{\mathcal{I}^{\prime}}$

2. $p^{-}\left((C)^{\mathcal{I}}\right)=\pi(\neg C)^{\mathcal{I}^{\prime}}$

Proof. We induct on the degree of $C$. If $C$ is atomic, then $\pi(C)=C$, and by definition $p^{+}\left(C^{\mathcal{I}}\right)$ $=C^{\mathcal{I}^{\prime}}=\pi(C)^{\mathcal{I}^{\prime}}$ and $p^{-}\left(C^{\mathcal{I}}\right)=C^{\prime \mathcal{I}^{\prime}}=\pi(\neg C)^{\mathcal{I}^{\prime}}$. If $C=\exists R$.Self, then $\pi(\exists R$.Self $)=\exists R$.Self, and by definition $(\exists R \text {.Self })^{\mathcal{I}^{\prime}}=p^{+}\left((\exists R \text {.Self })^{\mathcal{I}}\right)$. Furthermore, $\pi(\neg \exists R$.Self $)=C_{R . S e l f}$, and by definition $\left(C_{R \text {.Self }}\right)^{\mathcal{I}^{\prime}}=p^{-}\left((\exists R \text {.Self })^{\mathcal{I}}\right)$. If $C \in$ $N_{o}$, then $\pi(o)=o$, and by definition $o^{\mathcal{I}^{\prime}}=$ $p^{+}\left(o^{\mathcal{I}}\right)$. Furthermore, $\pi(\neg o)=C_{o}$, and by definition $\left(C_{o}\right)^{\mathcal{I}^{\prime}}=p^{-}\left(o^{\mathcal{I}}\right)$.

For the induction, we consider selected cases (the others are similar).

$$
\begin{aligned}
& \text { 1. } p^{+}\left((\mathbf{D} \sqcup \mathbf{E})^{\mathcal{I}}\right)=p^{+}(D) \cup p^{+}(E)=\pi(D)^{\mathcal{I}^{\prime}} \cup \\
& \pi(E)^{\mathcal{I}^{\prime}}=(\pi(D) \sqcup \pi(E))^{\mathcal{I}^{\prime}}=\pi(D \sqcup E)^{\mathcal{I}^{\prime}} . \\
& p^{-}\left((\mathbf{D} \sqcup \mathbf{E})^{\mathcal{I}}\right)=p^{-}(D) \cap p^{-}(E)=\pi(\neg D)^{\mathcal{I}^{\prime}} \cap \\
& \pi(\neg E)^{\mathcal{I}^{\prime}}=(\pi(\neg D) \sqcap \pi(\neg E))^{\mathcal{I}^{\prime}}=(\pi(\neg(D \sqcup \\
& E))^{\mathcal{I}^{\prime}}
\end{aligned}
$$

2. $p^{+}\left((\exists \mathbf{R} . \mathbf{D})^{\mathcal{I}}\right)=\left\{x \mid(\exists y)\left[(x, y) \in p^{+}\left(R^{\mathcal{I}}\right) \wedge\right.\right.$ $\left.\left.y \in p^{+}\left(D^{\mathcal{I}}\right)\right]\right\}=\left\{x \mid(\exists y)\left[(x, y) \in R^{\mathcal{I}^{\prime}} \wedge y \in\right.\right.$ $\left.\left.(\pi(D))^{\mathcal{I}^{\prime}}\right]\right\}=(\exists R . \pi(D))^{\mathcal{I}^{\prime}}=(\pi(\exists R . D))^{\mathcal{I}^{\prime}}$. $p^{-}\left((\exists \mathbf{R} . \mathbf{D})^{\mathcal{I}}\right)=$ $\left\{x \mid(\forall y)\left[(x, y) \in p^{+}\left(R^{\mathcal{I}}\right) \mapsto y \in p^{-}\left(D^{\mathcal{I}}\right)\right]\right\}=$ $\left\{x \mid(\forall y)\left[(x, y) \in R^{\mathcal{I}^{\prime}} \mapsto y \in(\pi(\neg D))^{\mathcal{I}^{\prime}}\right]\right\}=$ $(\forall R . \pi(\neg D))^{\mathcal{I}^{\prime}}=(\pi(\neg \exists R . D))^{\mathcal{I}^{\prime}}$.

3. $p^{+}\left((\leq \mathbf{n R} . \mathbf{D})^{\mathcal{I}}\right)=\left\{x \mid \sharp\left\{y \mid(x, y) \in p^{+}\left(R^{\mathcal{I}}\right) \wedge\right.\right.$ $\left.\left.y \notin p^{-}\left(D^{\mathcal{I}}\right)\right\} \leq n\right\}=$

$\left\{x \mid \sharp\left\{y \mid(x, y) \in R^{\mathcal{I}^{\prime}} \wedge y \notin \pi(\neg D)^{\mathcal{I}^{\prime}}\right\} \leq n\right\}=$ $\left\{x \mid \sharp\left\{y \mid(x, y) \in R^{\mathcal{I}^{\prime}} \wedge y \in\left(\Delta-\pi(\neg D)^{\mathcal{I}^{\prime}}\right)\right\} \leq n\right\}$ $=\left\{x \mid \sharp\left\{y \mid(x, y) \in R^{\mathcal{I}^{\prime}} \wedge y \in(\neg \pi(\neg D))^{\mathcal{I}^{\prime}}\right\} \leq n\right\}$ $=(\leq n R . \neg \pi(\neg D))^{\mathcal{I}^{\prime}}=(\pi(\leq n R . D))^{\mathcal{I}^{\prime}}$.

$p^{-}\left((\leq \mathbf{n R} . \mathbf{D})^{\mathcal{I}}\right)=\left\{x \mid \sharp\left\{y \mid(x, y) \in p^{+}\left(R^{\mathcal{I}}\right) \wedge\right.\right.$ $\left.\left.y \in p^{+}\left(D^{\mathcal{I}}\right)\right\} \geq(n+1)\right\}=\{x \mid \sharp\{y \mid(x, y) \in$ $\left.\left.R^{\mathcal{I}^{\prime}} \wedge y \in \pi(D)^{\mathcal{I}^{\prime}}\right\} \geq(n+1)\right\}=(\geq(n+$ 1)R. $\pi(D))^{\mathcal{I}^{\prime}}=(\pi(\neg \leq n R . D))^{\mathcal{I}^{\prime}}$.

The remaining cases, involving negation, all have the same form:

4. $p^{+}\left((\neg D)^{\mathcal{I}}\right)=p^{-}\left(D^{\mathcal{I}}\right)=\pi((\neg D))^{\mathcal{I}^{\prime}}$. $p^{-}\left((\neg D)^{\mathcal{I}}\right)=p^{+}\left(D^{\mathcal{I}}\right)=\pi(D)^{\mathcal{I}^{\prime}}=\pi(\neg \neg D)^{\mathcal{I}^{\prime}}$.

The below two lemmas are needed for the proof of Proposition 38

Lemma 63. For any 4-interpretation $\mathcal{I}$ and primed counterpart $\mathcal{I}^{\prime}$, if $S$ is the inverse of a role name $R$, then $p^{+}\left(S^{\mathcal{I}}\right)=S^{\mathcal{I}^{\prime}}$,

Proof. If $(a, b) \in p^{+}\left(S^{\mathcal{I}}\right)$, then $(b, a) \in p^{+}\left(R^{\mathcal{I}}\right)$. As such, $(b, a) \in R^{\mathcal{I}^{\prime}}$, and so $(a, b) \in S^{\mathcal{I}^{\prime}}$. If $(a, b) \in$ $S^{\mathcal{I}^{\prime}}$, then $(b, a) \in R^{\mathcal{I}^{\prime}}$, and so both $(b, a) \in p^{+}\left(R^{\mathcal{I}}\right)$ and $(a, b) \in p^{+}\left(S^{\mathcal{I}}\right)$.

Lemma 64. For any 4-interpretation $\mathcal{I}$ and primed counterpart $\mathcal{I}^{\prime}$,

$$
p^{+}\left(\left(R_{1} \circ \ldots \circ R_{n}\right)^{\mathcal{I}}\right)=\left(R_{1} \circ \ldots \circ R_{n}\right)^{\mathcal{I}^{\prime}} .
$$

Proof. (LR) If $(x, z) \in p^{+}\left(\left(R_{1} \circ \ldots \circ R_{n}\right)^{\mathcal{I}}\right)$, then there are $y_{1}, \ldots, y_{n-1}$ such that $\left(x, y_{1}\right) \in p^{+}\left(R_{1}^{\mathcal{I}}\right)$, $\left(y_{1}, y_{2}\right) \in p^{+}\left(R_{2}^{\mathcal{I}}\right), \ldots,\left(y_{n-1}, z\right) \in p^{+}\left(R_{n}^{\mathcal{I}}\right)$. Observe that for each $R_{i}, p^{+}\left(R_{i}^{\mathcal{I}}\right)=R_{i}^{\mathcal{I}^{\prime}}$ by definition of $\mathcal{I}^{\prime}$. And so $\left(x, y_{1}\right) \in R_{1}^{\mathcal{I}^{\prime}},\left(y_{1}, y_{2}\right) \in R_{2}^{\mathcal{I}^{\prime}}$, $\ldots,\left(y_{n-1}, z\right) \in R_{n}^{\mathcal{I}^{\prime}}$. As such, $(x, z) \in\left(R_{1} \circ\right.$ 
$\left.\ldots \circ R_{n}\right)^{\mathcal{I}^{\prime}}$. (RL) Suppose $(x, z) \in\left(R_{1} \circ \ldots \circ\right.$ $\left.R_{n}\right)^{\mathcal{I}^{\prime}}$. Then there exist $y_{1}, \ldots, y_{n-1}$ such that $\left(x, y_{1}\right) \in R_{1}^{\mathcal{I}^{\prime}},\left(y_{1}, y_{2}\right) \in R_{2}^{\mathcal{I}^{\prime}}, \ldots,\left(y_{n-1}, z\right) \in$ $R_{n}^{\mathcal{I}^{\prime}}$. For each $R_{i}, p^{+}\left(R_{i}^{\mathcal{I}}\right)=R_{i}^{\mathcal{I}^{\prime}}$ by definition of $\mathcal{I}^{\prime}$. And so $\left(x, y_{1}\right) \in p^{+}\left(R_{1}^{\mathcal{I}}\right),\left(y_{1}, y_{2}\right) \in$ $p^{+}\left(R_{2}^{\mathcal{I}}\right), \ldots,\left(y_{n-1}, z\right) \in p^{+}\left(R_{n}^{\mathcal{I}}\right)$. As such, $(x, z) \in$ $p^{+}\left(\left(R_{1} \circ \ldots \circ R_{n}\right)^{\mathcal{I}}\right)$.

Proposition 38, For any 4-interpretation $\mathcal{I}, \mathcal{I}$ is a 4-model of $\mathcal{S R O I Q 4}$ axiom $A$ iff its primed counterpart $\mathcal{I}^{\prime}$ is a 2 -model of $\pi(A)$.

Proof. (By cases):

1. $\mathbf{C}(\mathbf{a}): \pi(C(a))=\pi(C)(a)$. By Prop. 37, $a^{\mathcal{I}} \in$ $p^{+}\left(C^{\mathcal{I}}\right)$ iff $a^{\mathcal{I}^{\prime}} \in \pi(C)^{\mathcal{I}^{\prime}}$.

2. $\mathbf{A}=\mathbf{R}(\mathbf{a}, \mathbf{b}) . \pi(R(a, b))=R(a, b)$. Since $p^{+}\left(R^{\mathcal{I}}\right)=R^{\mathcal{I}^{\prime}}$ and $\left(a^{\mathcal{I}}, b^{\mathcal{I}}\right)=\left(a^{\mathcal{I}^{\prime}}, b^{\mathcal{I}^{\prime}}\right)$, $\left(a^{\mathcal{I}}, b^{\mathcal{I}}\right) \in p^{+}\left(R^{\mathcal{I}}\right)$ iff $\left(a^{\mathcal{I}^{\prime}}, b^{\mathcal{I}^{\prime}}\right) \in R^{\mathcal{I}^{\prime}}$

3. $\mathbf{A}=\neg \mathbf{R}(\mathbf{a}, \mathbf{b}) \cdot \pi(\neg R(a, b))=R^{\prime}(a, b)$. Since $p^{-}\left(R^{\mathcal{I}}\right)=R^{\prime \mathcal{I}^{\prime}}$ and $\left(a^{\mathcal{I}}, b^{\mathcal{I}}\right)=\left(a^{\mathcal{I}^{\prime}}, b^{\mathcal{I}^{\prime}}\right)$, $\left(a^{\mathcal{I}}, b^{\mathcal{I}}\right) \in p^{+}\left((\neg R)^{\mathcal{I}}\right)$ iff $\left(a^{\mathcal{I}^{\prime}}, b^{\mathcal{I}^{\prime}}\right) \in R^{\mathcal{I}^{\prime}}$.

4. $\mathbf{A}=(\mathbf{C} \sqsubset \mathbf{D})$ : $p^{+}\left(C^{\mathcal{I}}\right) \subseteq p^{+}\left(D^{\mathcal{I}}\right)$ iff $\pi(C)^{\mathcal{I}^{\prime}} \subseteq \pi(D)^{\mathcal{I}^{\prime}}$ iff $\mathcal{I}^{\prime}$ 2-satisfies $(\pi(C) \sqsubset \pi(D))$ iff $\mathcal{I}^{\prime}$ 2-satisfies $\pi(C \sqsubset D)$.

5. $\mathbf{A}=(\mathbf{C} \rightarrow \mathbf{D}):\left(p^{+}\left(C^{\mathcal{I}}\right) \subseteq p^{+}\left(D^{\mathcal{I}}\right)\right.$ and $\left.p^{-}\left(D^{\mathcal{I}}\right) \subseteq p^{-}\left(C^{\mathcal{I}}\right)\right)$ iff $\left(\pi(C)^{\mathcal{I}^{\prime}} \subseteq \pi(D)^{\mathcal{I}^{\prime}}\right.$ and $\left.\pi(\neg D)^{\mathcal{I}^{\prime}} \subseteq \pi(\neg C)^{\mathcal{I}^{\prime}}\right)$ iff ( $\mathcal{I}^{\prime}$ 2-satisfies $\{\pi(C) \sqsubseteq \pi(D), \pi(\neg D) \sqsubseteq \pi(\neg C)\}$ iff $\mathcal{I}^{\prime} 2$ satisfies $\pi(C \rightarrow D)$.

6. $\mathbf{A}=(\mathbf{C} \mapsto \mathbf{D}):\left(\Delta-p^{-}\left(C^{\mathcal{I}}\right)\right) \subseteq p^{+}\left(D^{\mathcal{I}}\right)$ iff $\left(\Delta-\pi(\neg C)^{\mathcal{I}^{\prime}}\right) \subseteq \pi(D)^{\mathcal{I}^{\prime}}$ iff $(\neg \pi(\neg C))^{\mathcal{I}^{\prime}} \subseteq$ $\pi(D)^{\mathcal{I}^{\prime}}$ iff $\mathcal{I}^{\prime}$ 2-satisfies $(\neg \pi(\neg C) \sqsubseteq \pi(D))$ iff $\mathcal{I}^{\prime}$ 2-satisfies $\pi(C \mapsto D)$.

7. $\mathbf{R}_{\mathbf{1}} \circ \ldots \circ \mathbf{R}_{\mathbf{n}} \sqsubset \mathbf{R}_{\mathbf{n}+\mathbf{1}}: \mathcal{I} 4$-satisfies $R_{1} \circ \ldots \circ$ $R_{n} \sqsubset R_{n+1}$ iff $p^{+}\left(\left(R_{1} \circ \ldots \circ R_{n}\right)^{\mathcal{I}}\right) \subseteq p^{+}\left(R_{n+1}^{\mathcal{I}}\right)$ iff $\left(R_{1} \circ\right.$ $\left.\ldots \circ R_{n}\right)^{\mathcal{I}^{\prime}} \subseteq R_{n+1}^{\mathcal{I}^{\prime}}$ iff $\mathcal{I}^{\prime}$ 2-satisfies $R_{1} \circ \ldots \circ$ $R_{n} \sqsubseteq R_{n+1}$ iff $\mathcal{I}^{\prime} 2$-satisfies $\pi\left(R_{1} \circ \ldots \circ R_{n} \sqsubset\right.$ $\left.R_{n+1}\right)$.

8. $\mathbf{A}=\operatorname{Ref}(\mathbf{R}): \mathcal{I}$ 4-satisfies $\operatorname{Re} f(R)$ iff $\{(x, x) \mid x \in \Delta\} \subseteq p^{+}\left(R^{\mathcal{I}}\right)$ iff $\{(x, x) \mid x \in \Delta\} \subseteq R^{\mathcal{I}^{\prime}}$ iff $\mathcal{I}^{\prime}$ 2-satisfies $\operatorname{Re} f(R)$ iff $\mathcal{I}^{\prime} 2$-satisfies $\pi(\operatorname{Ref}(R))$.

9. $\mathbf{A}=\operatorname{Irr}(\mathbf{R}): \mathcal{I}$ 4-satisfies $\operatorname{Irr}(R)$ iff $\{(x, x) \mid x \in \Delta\} \subseteq p^{-}\left(R^{\mathcal{I}}\right)$ iff $\{(x, x) \mid x \in \Delta\} \subseteq R^{\prime \mathcal{I}^{\prime}}$ iff $\mathcal{I}^{\prime}$ 2-satisfies $\operatorname{Ref}\left(R^{\prime}\right)$ iff $\mathcal{I}^{\prime}$ 2-satisfies $\pi(\operatorname{Irr}(R))$.

10. $\mathbf{A}=\operatorname{Dis}(\mathbf{R}, \mathbf{S}): \mathcal{I}$ 4-satisfies $\operatorname{Dis}(R, S)$ iff $\left(p^{+}\left(R^{\mathcal{I}}\right) \subseteq p^{-}\left(S^{\mathcal{I}}\right)\right.$ and $\left.p^{+}\left(S^{\mathcal{I}}\right) \subseteq p^{-}\left(R^{\mathcal{I}}\right)\right)$ iff $\left(R^{\mathcal{I}^{\prime}} \subseteq S^{\prime \mathcal{I}^{\prime}}\right.$ and $\left.S^{\mathcal{I}^{\prime}} \subseteq R^{\prime \mathcal{I}^{\prime}}\right)$ iff $\mathcal{I}^{\prime} 2$ satisfies $R \sqsubset S^{\prime}$ and $S \sqsubset R^{\prime}$ iff $\mathcal{I}^{\prime}$ 2-satisfies $\pi(\operatorname{Dis}(R, S))$.

Proposition 39 Let $K B$ be a $\mathcal{S R O} \mathcal{I} Q 4$ knowledge base. For any $\mathcal{S R O I} \mathcal{Q} 4$ axiom $A$ :

$$
K B=_{\mathcal{S R O} \mathcal{I} \mathcal{Q}_{4}} A \text { iff } \pi(K B) \models_{\mathcal{S R O} \mathcal{I} \mathcal{Q}} \pi(A) .
$$

Proof. (LR) Suppose $K B \models$ SROIQ $4 A$ and let $\mathcal{I}^{\prime}$ 2-satisfy $\pi(K B)$. Assume $w \log$ that $\mathcal{I}^{\prime}$ is the primed-counterpart of 4-interpretation $\mathcal{I}$ of $K B$. Since $\mathcal{I}^{\prime} 2$-satisfies $\pi(K B)$, by Prop. 38 $\mathcal{I}$ 4-satisfies $K B$ and hence $A$. By Prop. 38 $\mathcal{I}^{\prime}$ 2-satisfies $\pi(A)$. Generalizing on $\mathcal{I}^{\prime}$, it follows that $\pi(K B) \models \mathcal{S R O I \mathcal { Q }} \pi(A)$. (RL) Suppose $\pi(K B)=_{\mathcal{S R O I} \mathcal{Q}} \pi(A)$ and that $\mathcal{I}$ 4-satisfies $K B$. Then there is a 2-interpretation $\mathcal{I}^{\prime}$ that is the primed-counterpart of $\mathcal{I}$. By Prop. 38, since $\mathcal{I} 4$ satisfies $K B, \mathcal{I}^{\prime}$ 2-satisfies $\pi(K B)$ and so $\pi(A)$. By Prop 38, $\mathcal{I} 4$-satisfies $A$. Generalizing on $\mathcal{I}$, $K B=\mathcal{S R O I O}_{\mathcal{O} 4} A$.

\section{A.5. Tractable DLs}

Proof of Proposition 49. Let $K B \cup\{A\}$ be a set of Horn- $\mathcal{S H O I} \mathcal{O} \circ$ assertions, with all GCIs being $\sqsubset$-axioms. If $\pi_{\text {Horn }}(\pi(K B)) \models_{2} \pi_{\text {Horn }}(\pi(A))$, then $\pi(K B) \models_{2} \pi(A)$.

Proof. Suppose $\pi_{\mathrm{Horn}}(\pi(K B)) \quad \models_{2} \quad \pi_{\mathrm{Horn}}(\pi(A))$ and let $\mathcal{I}$ be a model of $\pi(K B)$. Since $B^{=}$does not appear in $\pi(K B)$, we may define a new interpretation $\mathcal{I}^{\prime}$ by extending $\mathcal{I}$ with $B^{=\mathcal{I}^{\prime}}=$ $\left(\neg B^{\prime}\right)^{\mathcal{I}}$. Clearly, $\mathcal{I}^{\prime}$ models every axiom of the form $B^{=} \sqcap B^{\prime} \sqsubset \perp$. Furthermore, since $B^{=\mathcal{I}^{\prime}}=$ $\left(\neg B^{\prime}\right)^{\mathcal{I}}$, the interpretations of each concept description in $\pi(K B)$ and corresponding description in $\pi_{\mathrm{Horn}}(\pi(K B))$ is the same, and so $\mathcal{I}^{\prime} 2$ satisfies $\pi_{\text {Horn }}(\pi(K B))$. Since $\pi_{\text {Horn }}(\pi(K B)) \models_{2}$ $\pi_{\text {Horn }}(\pi(A)), \mathcal{I}^{\prime} 2$-satisfies $\pi_{\text {Horn }}(\pi(A))$. However, since the interpretations of each concept description in $\pi(A)$ and corresponding description in $\pi_{\text {Horn }}(\pi(A))$ are the same, $\mathcal{I} 2$-satisfies $\pi(A)$.

Proof of Proposition 50. Every DL-Lite ${ }_{\text {core }}$ and DL-Lite $_{\mathcal{R}}$ knowledge base is 4-satisfiable.

Proof. Let $\Delta^{\mathcal{I}}=\{d\}$. Let $C^{\mathcal{I}}=\langle\{d\},\{d\}\rangle$ for each $C \in N_{C}$, and let $a^{\mathcal{I}}=d$ for each $a \in N_{I}$. For each role $R$, let $R^{\mathcal{I}}=\langle\{(d, d)\},\{(d, d)\}\rangle$. Observe 
that for each $C \in N_{C}, C^{\mathcal{I}}=(\neg C)^{\mathcal{I}}$, and for each role $R,(\neg R)^{\mathcal{I}}=R^{\mathcal{I}}$, and $(\exists R)^{\mathcal{I}}=(\neg \exists R)^{\mathcal{I}}=$ $\langle\{d\},\{d\}\rangle$. It is clear that each GCI, RIA, class assertion, and role assertion is 4 -satisfied.

For the remaining proofs about DL-Lite, we abbreviate $\pi_{\text {Lite }}$ to $\pi$. Let $\mathcal{I}$ be a four-valued interpretation. In order to accommodate the modified paraconsistent semantics for DL-Lite and its translation, we extend the definition of the primed counterpart $\mathcal{I}^{\prime}$ of $\mathcal{I}$ : For each role $R \in N_{R} \cup N_{R}^{-}$, $R^{=\mathcal{I}^{\prime}}=_{\text {def }} \Delta-p^{-}\left(R^{\mathcal{I}}\right)$.

Lemma 65. For any 4-interpretation $\mathcal{I}$, primed counterpart $\mathcal{I}^{\prime}$, and DL-Lite concept $C, p^{+}\left((C)^{\mathcal{I}}\right)$ $=\pi(C)^{\mathcal{I}^{\prime}}$, and $p^{-}\left((C)^{\mathcal{I}}\right)=\pi(\neg C)^{\mathcal{I}^{\prime}}$.

Proof. The cases for atomic concepts $A$ and their

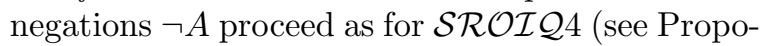
sition 37). We need only consider $\exists R$ and $\neg \exists R$.

1. If $C=\exists R$, then $\pi(\exists R)=\exists R$.

(a) $p^{+}\left((\exists R)^{\mathcal{I}}\right)=\left\{x \mid(\exists y)\left[(x, y) \in p^{+}\left(R^{\mathcal{I}}\right)\right]\right\}$.

However, $p^{+}\left(R^{\mathcal{I}}\right)=R^{\mathcal{I}^{\prime}}$, and so $p^{+}\left((\exists A)^{\mathcal{I}}\right)=(\exists R)^{\mathcal{I}^{\prime}}=\pi(\exists R)^{\mathcal{I}^{\prime}}$.

(b) $p^{-}\left((\exists R)^{\mathcal{I}}\right)=\left\{x \mid(\forall y)\left[(x, y) \in p^{-}\left(R^{\mathcal{I}}\right)\right]\right\}$. However, $R^{=\mathcal{I}^{\prime}}=\Delta-p^{-}\left(R^{\mathcal{I}}\right)$, and so $p^{-}\left((\exists R)^{\mathcal{I}}\right)=\left\{x \mid(\forall y)\left[(x, y) \notin R^{=\mathcal{I}^{\prime}}\right]\right\}$. So, $p^{-}\left((\exists R)^{\mathcal{I}}\right)=\left(\neg \exists R^{=}\right)^{\mathcal{I}^{\prime}}=\pi(\neg \exists R)^{\mathcal{I}^{\prime}}$.

2. If $C=\neg \exists R$, then $\pi(\neg \exists R)=\neg \exists R^{=}$.

(a) $p^{+}\left((\neg \exists R)^{\mathcal{I}}\right)=p^{-}\left((\exists R)^{\mathcal{I}}\right)=$ $\left\{x \mid(\forall y)\left[(x, y) \in p^{-}\left(R^{\mathcal{I}}\right)\right]\right\}$. Since $R^{=\mathcal{I}^{\prime}}=$ $\Delta-p^{-}\left(R^{\mathcal{I}}\right)$, it follows that $p^{+}\left((\neg \exists R)^{\mathcal{I}}\right)$ $=\left\{x \mid(\forall y)\left[(x, y) \notin R^{=\mathcal{I}^{\prime}}\right]\right\}$. And so $p^{+}\left((\neg \exists R)^{\mathcal{I}}\right)=\left(\neg \exists R^{=}\right)^{\mathcal{I}^{\prime}}$.

(b) $p^{-}\left((\neg \exists R)^{\mathcal{I}}\right)=\left\{x \mid(\exists y)\left[(x, y) \in p^{+}\left(R^{\mathcal{I}}\right)\right]\right\}$. However, $p^{+}\left(R^{\mathcal{I}}\right)=R^{\mathcal{I}^{\prime}}$, so $p^{-}\left((\neg \exists R)^{\mathcal{I}}\right)$ $=(\exists R)^{\mathcal{I}^{\prime}}=\pi(\exists R)^{\mathcal{I}^{\prime}}=\pi(\neg \neg \exists R)^{\mathcal{I}^{\prime}}$.

Lemma 66. Let $\mathcal{I}$ be a 4-interpretation and $\mathcal{I}^{\prime}$ its 2-counterpart. $\mathcal{I}$ is a 4-model of DL-Lite axiom $A$ iff $\mathcal{I}^{\prime}$ is a 2-model of $\pi(X)$.

Proof. For axioms of the form $C(a), R(a, b), C \sqsubset$ $D, C \rightarrow D, C \mapsto D$, the proof for Proposition 38 can be used (with Lemma 65 substituted appropriately). The cases for RIAs are given below.

1. $\mathbf{A}=(\mathbf{R} \sqsubset \mathbf{S}): \mathcal{I}$ 4-satisfies $R \sqsubset S$ iff $p^{+}\left(R^{\mathcal{I}}\right) \subseteq p^{+}\left(S^{\mathcal{I}}\right)$ iff $R^{\mathcal{I}^{\prime}} \subseteq S^{\mathcal{I}^{\prime}}$ iff $\mathcal{I}^{\prime} 2$ satisfies $R \sqsubseteq S$ iff $\mathcal{I}^{\prime} 2$-satisfies $\pi(R \sqsubset S)$.

2. $\mathbf{A}=(\mathbf{R} \sqsubset \neg \mathbf{S}): \mathcal{I}$ 4-satisfies $R \sqsubset \neg S$ iff $p^{+}\left(R^{\mathcal{I}}\right) \subseteq p^{-}\left(S^{\mathcal{I}}\right)$ iff $R^{\mathcal{I}^{\prime}} \subseteq S^{\prime \mathcal{I}^{\prime}}$ iff $\mathcal{I}^{\prime} \quad$ 2satisfies $R \sqsubseteq S^{\prime}$ iff $\mathcal{I}^{\prime} 2$-satisfies $\pi(R \sqsubset \neg S)$.
Proof of Proposition 52, For any DL-Lite ontology $K B$,

$$
K B=_{4} A \text { iff } \pi_{\text {Lite }}(K B) \models_{\text {DL-Lite }} \pi_{\text {Lite }}(A) .
$$

Proof. (LR) Suppose $K B=_{4} A$ and let $\mathcal{I}_{1}$ be a 2-model of $\pi(K B)$. For each role $R, R^{=\mathcal{I}_{1}} \subseteq$ $\Delta-R^{\prime \mathcal{I}_{1}}$. Since $R^{\prime}$ can only appear on the righthand side of an axiom of the form $S \sqsubseteq R^{\prime}, \mathcal{I}_{1}$ can be extended to a 2-model $\mathcal{I}^{\prime}$ of $\pi(K B)$ such that $R^{=\mathcal{I}^{\prime}}=\Delta-R^{\prime \mathcal{I}_{1}}$. Since $\mathcal{I}^{\prime}$ 2-satisfies $\pi(K B)$, then by Lemma 66 I $\mathcal{I}$-satisfies $K B$. And so $\mathcal{I}$ 4 -satisfies $A$. As such, $\mathcal{I}^{\prime} 2$-satisfies $\pi(A)$. We now show $\mathcal{I}_{1} 2$-satisfies $\pi(A)$, considering the form of A.

If $A$ is $C(a), R(a, b)$, GCI $C \sqsubset D$, RIA $C \sqsubset \exists R$, or RIA $R \sqsubseteq S$, where $R$ is atomic and $C, D$, and $S$ are atomic or simple negations, then since $\mathcal{I}$ and $\mathcal{I}_{1}$ differ only in the extension of $R^{=}$and $R^{=}$doesn't appear in $\pi(A), \mathcal{I}_{1} 2$-satisfies $\pi(A)$.

If $A=C \sqsubset \neg \exists R$, then $\pi(A)=\pi(C) \sqsubset \neg \exists R^{=}$, and so $\pi(C)^{\mathcal{I}^{\prime}} \subseteq\left(\neg \exists R^{=}\right)^{\mathcal{I}^{\prime}}$. Note that $\pi(C)^{\mathcal{I}^{\prime}}=$ $\pi(C)^{\mathcal{I}_{1}}$. Since $R^{=\mathcal{I}^{\prime}}=\Delta-R^{\prime \mathcal{I}_{1}},\{x \mid(\forall y)[(x, y) \notin$ $\left.\left.R^{=\mathcal{I}^{\prime}}\right]\right\}$ is a subset of $\left\{x \mid(\forall y)\left[(x, y) \notin R^{=\mathcal{I}_{1}}\right]\right\}$. From this, $\left(\neg \exists R^{=}\right)^{\mathcal{I}^{\prime}} \subseteq\left(\neg \exists R^{=}\right)^{\mathcal{I}_{1}}$. As such, $\pi(C)^{\mathcal{I}_{1}} \subseteq$ $\left(\neg \exists R^{=}\right)^{\mathcal{I}_{1}}$, and so $\mathcal{I}_{1}$ 2-satisfies $\pi(C \sqsubset \neg \exists R)$.

(RL) Suppose $\pi(K B) \models \pi(A)$ and $\mathcal{I}$ 4-satisfies $K B$. $\mathcal{I}$ corresponds to a 2 -interpretation $\mathcal{I}^{\prime}$ of $\pi(K B \cup\{A\})$. By Lemma 66, $\mathcal{I}^{\prime} 2$-satisfies $\pi(K B)$ and hence $\pi(A)$. By Lemma 66 I 4 -satisfies $A$.

\section{A.6. $E L P$}

Proof of Proposition 57, Let $K B$ be a $\supset$-rule base and $K B^{\prime}$ the result of applying one of $\mathrm{H} 1, \ldots, \mathrm{H} 4$, $\mathrm{B} 1, \ldots, \mathrm{B} 3$ to $K B . K B$ is 4 -satisfiable iff $K B^{\prime}$ is.

Proof. H2, H3, and B2 are trivial enough to omit. We consider the other cases. Let $r$ and $r^{\prime}$ denote the old and new rules, respectively. $K B^{\prime}$ potentially introduces new variables and atomic concepts. However, each interpretation for $K B^{\prime}$ extends one for $K B$, and each interpretation for $K B$ can be extended into one for $K B^{\prime}$.

H1: Since $\operatorname{body}(r)=\operatorname{body}\left(r^{\prime}\right), \mathcal{I}, \sigma_{\mathcal{I}} \not \models_{t} \operatorname{body}(r)$ iff $\mathcal{I}, \sigma_{\mathcal{I}} \not \forall_{t}$ body $\left(r^{\prime}\right)$. Obviously, $t^{\mathcal{I}} \in p^{+}\left((C \sqcap D)^{\mathcal{I}}\right)$ iff $\mathcal{I}, \sigma_{\mathcal{I}} \models_{t}(C(t) \wedge D(t))$. The rest of head $\left(r^{\prime}\right)$ is unchanged from $r$, and so $\mathcal{I}, \sigma_{\mathcal{I}} \models_{t}$ head $(r)$ iff $\mathcal{I}, \sigma_{\mathcal{I}} \models_{t}$ head $\left(r^{\prime}\right)$. So, $\mathcal{I}, \sigma_{\mathcal{I}} \models_{t} r$ iff $\mathcal{I}, \sigma_{\mathcal{I}} \models_{t} r^{\prime}$. 
H4: Let $s=A(x) \supset C(x)$ be the axiom added. (LR) Suppose $\mathcal{I}, \sigma_{\mathcal{I}} \models_{t} r$, and let $\mathcal{I}^{\prime}$ be obtained by extending $\mathcal{I}$ so that $A^{\mathcal{I}^{\prime}}=C^{\mathcal{I}}$ (ensuring $\mathcal{I}^{\prime}, \sigma_{\mathcal{I}^{\prime}} \models_{t} s$ ). Since $A^{\mathcal{I}^{\prime}}=C^{\mathcal{I}}$, it must be that $\mathcal{I}, \sigma_{\mathcal{I}} \models_{t} r^{\prime}$. (RL) Suppose $\mathcal{I}, \sigma_{\mathcal{I}}=_{t} r^{\prime}$ and $\mathcal{I}, \sigma_{\mathcal{I}} \models_{t} s$ for some $\mathcal{I}$. If $\mathcal{I}, \sigma_{\mathcal{I}} \models_{t} \operatorname{body}\left(r^{\prime}\right)$, then $\mathcal{I}, \sigma_{\mathcal{I}}=_{t} r$. If $\mathcal{I}, \sigma_{\mathcal{I}} \models_{t}$ $\operatorname{head}\left(r^{\prime}\right)$, then $\mathcal{I}, \sigma_{\mathcal{I}} \models_{t} \exists R . A(t)$, and since $\mathcal{I}, \sigma_{\mathcal{I}}=_{t} s$, it follows that $\mathcal{I}, \sigma_{\mathcal{I}}={ }_{t} \exists R . C(t)$. Since the rest of head $\left(r^{\prime}\right)$ matches head $(r)$, $\mathcal{I}, \sigma_{\mathcal{I}} \models_{t} \operatorname{head}(r)$, and so $\mathcal{I}, \sigma_{\mathcal{I}} \models_{t} r$.

B1: Suppose $\mathcal{I}, \sigma_{\mathcal{I}} \models_{t} r$. Then either $\mathcal{I}, \sigma_{\mathcal{I}} \forall_{t}$ $\operatorname{body}(r)$ or else $\mathcal{I}, \sigma_{\mathcal{I}} \models_{t} \operatorname{head}(r)$. If $\mathcal{I}, \sigma_{\mathcal{I}} \models_{t}$ $\operatorname{head}(r)$, then $\mathcal{I}, \sigma_{\mathcal{I}} \models_{t} \operatorname{head}\left(r^{\prime}\right)$, and so $\mathcal{I}, \sigma_{\mathcal{I}} \models_{t} r^{\prime}$. If $\mathcal{I}, \sigma_{\mathcal{I}} \not \models_{t} \operatorname{body}(r)$, then $\mathcal{I}, \sigma_{\mathcal{I}} \not \models_{t}$ $A$ for some $A \in \operatorname{body}(r)$. If $A \in \operatorname{body}\left(r^{\prime}\right)$, then $\mathcal{I}, \sigma_{\mathcal{I}} \forall_{t} \operatorname{body}\left(r^{\prime}\right)$. If $A \notin \operatorname{body}\left(r^{\prime}\right)$, then $A=(C \sqcap D)(t)$. As such, either $\mathcal{I}, \sigma_{\mathcal{I}} \nvdash_{t} C(t)$ or else $\mathcal{I}, \sigma_{\mathcal{I}} \nvdash_{t} D(t)$. Either way, $\mathcal{I}, \sigma_{\mathcal{I}} \not \nvdash_{t}$ $C(t) \wedge D(t)$. And so $\mathcal{I}, \sigma_{\mathcal{I}} \not \neq_{t} \operatorname{body}\left(r^{\prime}\right)$, which implies $\mathcal{I}, \sigma_{\mathcal{I}}=_{t} r^{\prime}$.

The other direction proceeds similarly.

B3: $\operatorname{head}(r)=\operatorname{head}\left(r^{\prime}\right)$, and so $\mathcal{I}, \sigma_{\mathcal{I}} \models_{t}$ head $(r)$ iff $\mathcal{I}, \sigma_{\mathcal{I}}=_{t}$ head $\left(r^{\prime}\right)$. We consider the bodies: (LR) Suppose $\mathcal{I}, \sigma_{\mathcal{I}} \nvdash_{t} \operatorname{body}(r)$. If this is due to an unrelated atom (not $\exists R . C(t)$ ), then $\mathcal{I}, \sigma_{\mathcal{I}} \not \nvdash_{t} \operatorname{body}\left(r^{\prime}\right)$. So suppose $\mathcal{I}, \sigma_{\mathcal{I}} \not \nvdash_{t}$ $\exists R . C(t)$. By definition, $t^{\mathcal{I}} \notin\{z \mid(\exists y)[(z, y) \in$ $p^{+}\left(R^{\mathcal{I}}\right)$ and $\left.\left.y \in p^{+}\left(C^{\mathcal{I}}\right)\right]\right\}$. So for all $d \in \Delta^{\mathcal{I}}$, either $\left(t^{\mathcal{I}}, d\right) \notin p^{+}\left(R^{\mathcal{I}}\right)$ or else $d \notin p^{+}\left(C^{\mathcal{I}}\right)$. In other words, for all $d$, either $\mathcal{I}, \sigma_{\mathcal{I}}[x / d] \forall_{t}$ $C(x)$ or else $\mathcal{I}, \sigma_{\mathcal{I}}[x / d] \quad \forall_{t} R(t, x)$. Either way, $\mathcal{I}, \sigma_{\mathcal{I}} \neq_{t} \operatorname{body}\left(r^{\prime}\right)$. (RL) Now suppose $\mathcal{I}, \sigma_{\mathcal{I}} \nvdash_{t} \operatorname{body}\left(r^{\prime}\right)$, and assume that for all $d \in \Delta^{\mathcal{I}}$, either $\mathcal{I}, \sigma_{\mathcal{I}}[x / d] \forall_{t} C(x)$ or else $\mathcal{I}, \sigma_{\mathcal{I}}[x / d] \quad \forall_{t} R(t, x)$. Either way, $\mathcal{I}, \sigma_{\mathcal{I}} \nvdash_{t}$ $\exists R$.C. And so $\mathcal{I}, \sigma_{\mathcal{I}} \not_{t} \operatorname{body}(r)$.

Proof of Proposition 58. If $\mathcal{P}$ is a well-formed formula of ELP, $\mathcal{I}$ is a 4-interpretation of $\mathcal{P}$, and $\mathcal{I}^{\prime}$ is the primed counterpart of $\mathcal{I}$, then for any assignment $\sigma_{\mathcal{I}}, \mathcal{I}, \sigma_{\mathcal{I}}={ }_{t} \mathcal{P}$ iff $\mathcal{I}, \sigma_{\mathcal{I}^{\prime}}=\pi(\mathcal{P})$.

Proof. Let $\mathcal{I}$ be a 4-interpretation and $\mathcal{I}^{\prime}$ its primed counterpart. We induct on the degree of $\mathcal{P}$, considering selected cases. The rest are similar.

1. $\mathbf{C}(\mathbf{t}): \mathcal{I}, \sigma_{\mathcal{I}} \models_{t} C(t)$ iff $\sigma_{\mathcal{I}}(t) \in p^{+}\left(C^{\mathcal{I}}\right)$ iff $\sigma_{\mathcal{I}}(t) \in \pi(C)^{\mathcal{I}^{\prime}}$ iff $\mathcal{I}^{\prime}, \sigma_{\mathcal{I}} \models \pi(C)(t)$.

2. $\neg \mathbf{R}\left(\mathbf{t}_{\mathbf{1}}, \mathbf{t}_{\mathbf{2}}\right): \mathcal{I}, \sigma_{\mathcal{I}}=_{t} \neg R\left(t_{1}, t_{2}\right)$ iff $\left(\sigma_{\mathcal{I}}\left(t_{1}\right), \sigma_{\mathcal{I}}\left(t_{2}\right)\right) \in p^{-}\left(R^{\mathcal{I}}\right)$ iff
$\left(\sigma_{\mathcal{I}}\left(t_{1}\right), \sigma_{\mathcal{I}}\left(t_{2}\right)\right) \in R^{\prime \mathcal{I}}$ iff $\left(\sigma_{\mathcal{I}}\left(t_{1}\right), \sigma_{\mathcal{I}}\left(t_{2}\right)\right) \in$ $\pi(\neg R)^{\mathcal{I}^{\prime}}$ iff $\mathcal{I}^{\prime}, \sigma_{\mathcal{I}}=\pi(\neg R)\left(t_{1}, t_{2}\right)$.

3. $(\mathcal{P} \wedge \mathcal{Q}): \mathcal{I}, \sigma_{\mathcal{I}} \models_{t}(\mathcal{P} \wedge \mathcal{Q})$ iff $\left(\mathcal{I}, \sigma_{\mathcal{I}} \models_{t} \mathcal{P}\right.$ and $\left.\mathcal{I}, \sigma_{\mathcal{I}} \models_{t} \mathcal{Q}\right)$ iff $\left(\mathcal{I}^{\prime}, \sigma_{\mathcal{I}}=\pi(\mathcal{P})\right.$ and $\mathcal{I}, \sigma_{\mathcal{I}^{\prime}} \models_{t}$ $\pi(\mathcal{Q}))$ iff $\mathcal{I}^{\prime}, \sigma_{\mathcal{I}} \models \pi(\mathcal{P} \wedge \mathcal{Q})$.

4. $(\mathcal{P} \supset \mathcal{Q}): \mathcal{I}, \sigma_{\mathcal{I}} \models_{t}(\mathcal{P} \supset \mathcal{Q})$ iff $\left(\mathcal{I}, \sigma_{\mathcal{I}} \models_{t} \mathcal{P}\right.$ or $\left.\mathcal{I}, \sigma_{\mathcal{I}} \models_{t} \mathcal{Q}\right)$ iff $\left(\mathcal{I}^{\prime}, \sigma_{\mathcal{I}} \vDash \pi(\mathcal{P})\right.$ or $\mathcal{I}, \sigma_{\mathcal{I}^{\prime}} \models_{t}$ $\pi(\mathcal{Q}))$ iff $\left(\mathcal{I}^{\prime}, \sigma_{\mathcal{I}}=\pi(\mathcal{P}) \mapsto \pi(\mathcal{Q})\right)$ iff $\mathcal{I}^{\prime}, \sigma_{\mathcal{I}} \models$ $\pi(\mathcal{P} \supset \mathcal{Q})$.

5. $(\forall \mathbf{x}) \mathcal{P}, x$ not safe: $\mathcal{I}, \sigma_{\mathcal{I}} \models_{t}(\forall x) \mathcal{P}$ iff $\mathcal{I}, \sigma_{\mathcal{I}}[x / d] \models_{t} \mathcal{P}$ for all $d \in \Delta$ iff $\mathcal{I}^{\prime}, \sigma_{\mathcal{I}}[x / d] \models$ $\mathcal{P}$ for all $d \in \Delta$ iff $\mathcal{I}^{\prime}, \sigma_{\mathcal{I}}=(\forall x) \mathcal{P}$.

6. $(\forall \mathbf{x}) \mathcal{P}, x$ safe: $\mathcal{I}, \sigma_{\mathcal{I}}=_{t}(\forall x) \mathcal{P}$ iff $\mathcal{I}, \sigma_{\mathcal{I}}[x / d] \models{ }_{t} \mathcal{P}$ for all named $d \in \Delta$ iff $\mathcal{I}^{\prime}, \sigma_{\mathcal{I}}[x / d] \models \mathcal{P}$ for all named $d \in \Delta$ iff $\mathcal{I}^{\prime}, \sigma_{\mathcal{I}}=(\forall x) \mathcal{P}$.

Proof of Proposition 59, Let $K B$ be an ELP rule base and $\mathcal{P}$ a well-formed formula of ELP. $K B \mid{ }_{E L P 4} \mathcal{P}$ iff $\pi(K B) \models_{E L P} \pi(\mathcal{P})$.

Proof. (LR) Suppose $K B \models_{E L P 4} \mathcal{P}$ and let $\mathcal{I}^{\prime}$ be a model of $\pi(K B)$ and $\sigma_{\mathcal{I}^{\prime}}$ an assignment for $\mathcal{I}^{\prime}$. Assume $w \log$ that $\mathcal{I}^{\prime}$ is the primedcounterpart of a 4-interpretation $\mathcal{I}$. Since $\mathcal{I}^{\prime}$ models $\pi(K B), \mathcal{I}^{\prime}, \sigma_{\mathcal{I}^{\prime}} \models_{E L P} \pi(K B)$. From Prop. $58 . \mathcal{I}, \sigma_{\mathcal{I}} \models_{E L P 4} K B$ and so $\mathcal{I}, \sigma_{\mathcal{I}} \models_{E L P 4} \mathcal{P}$. From Prop. 58, $\mathcal{I}^{\prime}, \sigma_{\mathcal{I}^{\prime}} \models_{E L P} \pi(\mathcal{P})$. (RL) Suppose $\pi(K B) F_{E L P} \pi(\mathcal{P})$ and let $\mathcal{I} 4$-model $K B$ and let $\sigma_{\mathcal{I}}$ be an assignment for $\mathcal{I}$. Let $\mathcal{I}^{\prime}$ be the primed-counterpart of $\mathcal{I}$. Since $\mathcal{I}$ 4-satisfies $K B$, $\mathcal{I}, \sigma_{\mathcal{I}}={ }_{E L P 4} K B$. From Prop. $58, \mathcal{I}^{\prime}, \sigma_{\mathcal{I}^{\prime}}=_{E L P}$ $\pi(K B)$ and so $\mathcal{I}^{\prime}, \sigma_{\mathcal{I}^{\prime}} \models_{E L P} \pi(\mathcal{P})$. From Prop. 58 $\mathcal{I}, \sigma_{\mathcal{I}}=_{E L P 4} \mathcal{P}$.

\section{References}

[1] Carlos E. Alchourrón, Peter Gärdenfors, and David Makinson. On the Logic of Theory Change: Partial Meet Contraction and Revision Functions, Journal of Symbolic Logic, 50(2): 510-530, 1985.

[2] Marcelo Arenas, Leopoldo Bertossi, and Jan Chomicki. Consistent query answers in inconsistent databases. Proceedings of the eighteenth ACM SIGMODSIGACT-SIGART symposium on Principles of database systems (PODS '99), pages 68-79. ACM, 1999.

[3] Ofer Arieli. Paraconsistent reasoning and preferential entailments by signed quantified boolean formulae. ACM Transactions on Computational Logic, 8(3):18, 2007. 
[4] Ofer Arieli. On the application of the disjunctive syllogism in paraconsistent logics based on four states of information. Proceedings of the Twelfth International Conference on the Principles of Knowledge Representation and Reasoning (KR 2010), Toronto, Ontario, Canada, May 9-13, 2010. AAAI Press, 2010.

[5] Ofer Arieli and Arnon Avron. Reasoning with logical bilattices. Journal of Logic, Language and Information, 5(1):25-63, 1996.

[6] Ofer Arieli and Arnon Avron. The value of the four values. Artificial Intelligence, 102(1):97-141, 1998.

[7] Ofer Arieli and Marc Denecker. Reducing preferential paraconsistent reasoning to classical entailment. Journal of Logic and Computation, 13(4):557-580, 2003.

[8] Franz Baader, Sebastian Brandt, Carsten Lutz. Pushing the $\mathcal{E} \mathcal{L}$ envelope. Proceedings of the 19th international joint conference on artificial intelligence (IJCAI-05), Edinburgh, UK, 2005, pages 364-369. Morgan-Kaufmann Publishers, 2005.

[9] Nuel D. Belnap. How a computer should think. G. Ryle, editor, Contemporary Aspects of Philosophy. Oriel Press, 1977.

[10] Nuel D. Belnap. A useful four-valued logic. G. Epstein and J. Dunn, editors, Modern uses of multiple valued logics, pages 8-37. D. Reidel, Dordrecht, 1977.

[11] Leopoldo E. Bertossi, Anthony Hunter and Torsten Schaub, editors. Inconsistency Tolerance. LNCS vol. 3300. Springer, 2005.

[12] Diego Calvanese, Giuseppe De Giacomo, Domenico Lembo, Maurizio Lenzerini, and Riccardo Rosati. Tractable reasoning and efficient query answering in description logics: The DL-Lite family. Journal of $\mathrm{Au}$ tomated Reasoning, 39(3):385-429, 2007.

[13] Andrea Calì, Domenico Lembo, and Riccardo Rosati. On the decidability and complexity of query answering over inconsistent and incomplete databases. Proceedings of the twenty-second ACM SIGMOD-SIGACTSIGART symposium on Principles of database systems (PODS '03), pages 260-271. ACM, 2003.

[14] Walter A. Carnielli, Marcelo Coniglio, and João Marcos. Logics of Formal Inconsistency. D. Gabbay and F. Guenthner, editors, Handbook of Philosophical Logic (2nd ed.), Volume 14, pages 15-107. SpringerVerlag, 2005.

[15] Walter A. Carnielli and João Marcos. A taxonomy of $\mathcal{C}$-Systems. Paraconsistency: The Logical Way to the Inconsistent. Proceedings of the II World Congress on Paraconsistency (WCP'2000), pages 1-94. Marcel Dekker, 2001.

[16] Newton C. A. da Costa. On the theory of inconsistent formal systems. Notre Dame Journal of Formal Logic 15(4): 497-510, 1974.

[17] Newton C. A. da Costa, Jean-Yves Béziau, and Otávio Bueno. Professor Newton C.A. da Costa awarded Nicholas Copernicus University medal of merit. Logic and Logical Philosophy 7: 7-10, 1999

[18] Newton C. A. da Costa, D. Krause, and Otávio Bueno. Paraconsistent logics and paraconsistency: Technical and philosophical developments. CLE ePrints 4(3), May 2004. http://www.cle.unicamp.br/ e-prints/vol_4,n_3,2004.html.
[19] Carlos Viegas Damásio and Luís Moniz Pereira. A Paraconsistent semantics with contradiction support detection. Proceedings of the 4 th International Conference on Logic Programming and Nonmonotonic Reasoning (LPNMR '97), Dagstuhl Castle, Germany, July 28-31, 1997, pages 224-243. Springer-Verlag, 1997.

[20] Carlos Viegas Damásio and Luís Moniz Pereira. A survey of paraconsistent semantics for logic programs. Philippe Besnard, Anthony Hunter, Dov M. Gabbay, and Philippe Smets, editors, Handbook of Defeasible Reasoning and Uncertainty Management Systems, pages 241-320. Kluwer Academic Publishers, 1998.

[21] Sandra de Amo and Mônica Sakuray Pais. A paraconsistent logic programming approach for querying inconsistent databases. International Journal of Approximate Reasoning 46(2): 366-386, 2007.

[22] Jon Doyle. A Truth Maintenance System. Artificial Intelligence, 12(3): 231-272, 1979.

[23] L. Dubikajtis. The life and works of stansisław jaśkowski. Studia Logica 34(2), 109-116, 1975.

[24] Peter Gärdenfors and David Makinson. Revision of knowledge systems using epistemic entrenchment. Moshe Y. Vardi, editor, Proceedings of the Second Conference on Theoretical Aspects of Reasoning about Knowledge (TARK'88), pages 83-95. Morgan Kaufmann Publishers, 1988.

[25] Michael Gelfond and Vladimir Lifschitz. Classical negation in logic programs and disjunctive databases. New Generation Computing, 9(3/4): 365-386, 1991

[26] Allen Van Gelder, Kenneth A. Ross, and John S. Schlipf. The well-founded semantics for general logic programs. Journal of the ACM, 38(3): 620-650, 1991.

[27] Yuanbo Guo, Zhengxiang Pan, and Jeff Heflin. LUBM: A benchmark for OWL knowledge base systems. Web Semantics 3(2-3): 158-182, 2005.

[28] Benjamin N. Grosof, Ian Horrocks, Raphael Volz, and Stefan Decker. Description logic programs: combining logic programs with description logic. Proceedings of the 12th International World Wide Web Conference (WWW-03), Budapest, Hungary, pages 48-57. ACM, 2003.

[29] Qing Han and Zuoquan Lin. Paraconsistent default reasoning. James P. Delgrande and Torsten Schaub, editors, Proceedings of the 10th International Workshop on Non-Monotonic Reasoning (NMR 2004), Whistler, Canada, June 6-8, 2004, pages 197-203. 2004.

[30] Pascal Hitzler, Markus Krötzsch, Bijan Parsia, Peter Patel-Schneider, and Sebastian Rudolph, editors. OWL 2 Web Ontology Language: Primer. W3C Recommendation, 27 October 2009. Available at http: //www.w3.org/TR/owl2-primer/

[31] Pascal Hitzler, Markus Krötzsch, and Sebastian Rudolph. Foundations of Semantic Web Technologies. Chapman \& Hall/CRC, 2009.

[32] Pascal Hitzler and Frank van Harmelen. A reasonable Semantic Web. Semantic Web Journal 1(1-2): 39-44, 2010.

[33] Matthew Horridge and Sean Bechhofer. The OWL API: A Java API for OWL ontologies. Semantic Web 
Journal 2(1): 1-21, 2011.

[34] Ian Horrocks, Oliver Kutz, and Ulrike Sattler. The even more irresistible $\mathcal{S} \mathcal{R O} \mathcal{I} \mathcal{Q}$. Patrick Doherty, John Mylopoulos, and Christopher A. Welty, editors, Proceedings, Tenth International Conference on Principles of Knowledge Representation and Reasoning, Lake District of the United Kingdom, June 2-5, 2006, pages 57-67. AAAI Press, 2006.

[35] Ian Horrocks and Peter F. Patel-Schneider. Reducing OWL entailment to description logic satisfiability. Journal of Web Semantics 1(4): 345-457, 2004.

[36] Zhisheng Huang, Frank Van Harmelen, and Annette ten Teije. Reasoning with inconsistent ontologies. Proceedings of the 19th international joint conference on artificial intelligence (IJCAI'O5), pages 454-459. Morgan Kaufmann Publishers Inc, 2005.

[37] Anthony Hunter. Reasoning with contradictory information using quasi-classical logic. Journal of Logic and Computation, 10(5):677-703, 2000.

[38] Stanisław Jaśkowski. Propositional calculus for contradictory deductive systems. Studia Logica 24: 143-160, 1969

[39] Aditya Kalyanpur, Bijan Parsia, Evren Sirin, and James A. Hendler. Debugging unsatisfiable classes in OWL ontologies. Journal of Web Semantics, 3(4): 268-293, 2005.

[40] Aditya Kalyanpur, Bijan Parsia, Matthew Horridge, and Evren Sirin. Finding all justifications of OWL DL entailments. Proceedings of the 6th international The semantic web and 2nd Asian conference on Asian semantic web conference (ISWC'07/ASWC'07), pages 267-280. Springer-Verlag, 2007.

[41] Norihiro Kamide. Paraconsistent description logics revisited. Volker Haarslev, David Toman, and Grant Weddell, editors, Proceedings of the 23rd International Workshop on Description Logics (DL2010), Waterloo, Canada, 2010. Volume 573 of CEUR Workshop Proceedings. CEUR-WS.org, 2010.

[42] Stephen Kleene. Introduction to Metamathematics. North-Holland, 1952.

[43] Markus Krötzsch, Sebastian Rudolph, and Pascal Hitzler. Complexity boundaries for Horn description logics. Proceedings of the Twenty-Second AAAI Conference on Artificial Intelligence, July 22-26, 2007, Vancouver, British Columbia, Canada, pages 452-457. AAAI Press, 2007

[44] Markus Krötzsch, Sebastian Rudolph, and Pascal Hitzler. ELP: Tractable rules for OWL 2. A. Sheth, S. Staab, M. Dean, M. Paolucci, D. Maynard, T. Finin, and K. Thirunarayan, editors, Proceedings of the 7th International Semantic Web Conference (ISWC-08), Karlsruhe, Germany, October 26-30, 2008, volume 5318 of Lecture Notes in Computer Science, pages 649-664. Springer, 2008.

[45] Andreas Christian Lang. Four-valued logics for paraconsistent reasoning. Diplomarbeit. Dresden University of Technology, 2006.

[46] Yue Ma and Pascal Hitzler. Paraconsistent reasoning for OWL 2. Axel Polleres and Terrance Swift, editors, Proceedings of the Third International Conference on Web Reasoning and Rule Systems (RR 2009), Chan- tilly, VA, USA, October 25-26, 2009, volume 5837 of Lecture Notes in Computer Science, pages 197-211. Springer Berlin/Heidelberg, 2009.

[47] Yue Ma, Pascal Hitzler, and Zuoquan Lin. Paraconsistent reasoning with OWL - algorithms and the ParOWL reasoner. Technical Report 1390. AIFB, University of Karlsruhe, Germany, 2006.

[48] Yue Ma, Pascal Hitzler, and Zuoquan Lin. Algorithms for paraconsistent reasoning with OWL. Enrico Franconi, Michael Kifer, and Wolfgang May, editors, Proceedings of the 4th Europoean Semantic Web Conference (ESWC 2007), Innsbruck, Austria, June 2007, volume 4519 of Lecture Notes in Computer Science, pages 399-413. Springer, 2007.

[49] Yue Ma, Pascal Hitzler, and Zuoquan Lin. Paraconsistent resolution for four-valued description logics. Diego Calvanese, Enrico Franconi, Volker Haarslev, Domenico Lembo, Boris Motik, Anni-Yasmin Turhan, and Sergio Tessaris, editors, Proceedings of the 2007 International Workshop on Description Logics (DL2007), Brixen-Bressanone, Italy, 8-10 June, 2007, volume 250 of CEUR Workshop Proceedings. CEURWS.org, 2007.

[50] Yue Ma, Pascal Hitzler, and Zuoquan Lin. Paraconsistent reasoning for expressive and tractable description logics. Franz Baader, Carsten Lutz, and Boris Motik, editors, Proceedings of the 21st International Workshop on Description Logics (DL 2008), Dresden, Germany, May 2008, volume 353 of CEUR Workshop Proceedings. CEUR-WS.org, 2008.

[51] Yue Ma, Zuoquan Lin, and Zhangang Lin. Inferring with inconsistent OWL DL ontology: A multi-valued logic approach. Torsten Grust, et al., editors, Current Trends in Database Technology - EDBT 2006, volume 4254 of Lecture Notes in Computer Science, pages 535-553. Springer, 2006.

[52] Yue Ma, Guilin Qi, Pascal Hitzler, and Zuoquan Lin. Measuring inconsistency for description logics based on paraconsistent semantics. Khaled Mellouli, editor, Proceedings of the 9th European Conference on Symbolic and Quantitative Approaches to Reasoning with Uncertainty, (ECSQARU 2007), Hammamet, Tunisia, October 31 - November 2, 2007, volume 4724 of Lecture Notes in Computer Science, pages 30-41. Springer, 2007.

[53] Frederick Maier. Extending paraconsistent $\mathcal{S} \mathcal{R O} \mathcal{I} \mathcal{Q}$. Pascal Hitzler and Thomas Lukasiewicz, editors, Proceedings of the Fourth International Conference on Web Reasoning and Rule Systems (RR 2010), Bressanone/Brixen, Italy, September 22-24, 2010, volume 6333 of Lecture Notes in Computer Science, pages 118-132. Springer-Verlag Berlin/Heidelberg, 2010.

[54] Carlo Meghini, Fabrizio Sebastiani, Umberto Straccia, and Consiglio Nazionale. MIRLOG: A logic for multimedia information retrieval. Fabio Crestani, Mounia Lalmas and Cornelis J. van Rijsbergen, editors, Information Retrieval: Uncertainty and Logics. Advanced models for the representation and retrieval of information, pages 151-185. Kluwer Academic Publishing, 1998.

[55] Carlo Meghini and Umberto Straccia. A relevance ter- 
minological logic for information retrieval. SIGIR '96: Proceedings of the 19th annual international ACM SIGIR conference on Research and development in in formation retrieval, pages 197-205. ACM, 1996.

[56] Boris Motik. Reasoning in description logics using resolution and deductive databases. PhD Thesis, University of Karlsruhe, Germany, 2006.

[57] Boris Motik, Bernardo Cuenca Grau, Ian Horrocks, Zhe $\mathrm{Wu}$, Achille Fokoue, and Carsten Lutz, editors. OWL 2 Web Ontology Language: Profiles. W3C Recommendation 27 October, 2009. Available at http:// www.w3.org/TR/2009/REC-owl2-profiles-20091027/

[58] Donald Nute. Defeasible logic. D. Gabbay and C. Hogger, editors, Handbook of logic in artificial intelligence and logic programming (vol. 3): nonmonotonic reasoning and uncertain reasoning, pages 353-395. Oxford University Press, 1994

[59] Peter Patel-Schneider. A hybrid, decidable, logicbased knowledge representation system. Computational Intelligence, 3(1):64-77, 1987.

[60] Peter Patel-Schneider. A four-valued semantics for terminological logics. Articial Intelligence, 38(3):319351, 1989.

[61] Karl Popper. What is dialectic? Mind, 49(196):403426, 1940.

[62] Graham Priest. Logic of paradox. Journal of Philosophical Logic, 8(1):219-241, 1979.

[63] Raymond Reiter. A Logic for default reasoning. Artificial Intelligence, 13:81-132, 1980.

[64] Raymond Reiter. A theory of diagnosis from first principles. Artificial Intelligence, 32:57-95, 1987.

[65] Sebastian Rudolph, Markus Krötzsch, and Pascal Hitzler. Cheap Boolean role constructors for description logics. S. Hölldobler et al., editors, Proceedings of the 11th European Conference on Logics in Artificial Intelligence (JELIA'08), volume 5293 of LNAI, pages 362-374. Springer, 2008.

[66] Stefan Schlobach and Ronald Cornet. Non-standard reasoning services for the debugging of description logic terminologies. Georg Gottlob and Toby Walsh, editors, Proceedings of the Eighteenth International Joint Conference on Artificial Intelligence (IJCAI03), Acapulco, Mexico, August 9-15, 2003, pages 355360. Morgan Kaufmann, 2003.

[67] Evren Sirin, Bijan Parsia, Bernardo Cuenca Grau, Aditya Kalyanpur, and Yarden Katz. Pellet: A practical OWL-DL reasoner. Journal of Web Semantics
$5(2): 51-53,2007$

[68] Umberto Straccia. Document retrieval by relevance terminological logics. Proceedings of the Final Workshop on Multimedia Information Retrieval (MIRO-95) Glasgow, Scotland, UK, 18-20 September 1995, pages 128-133. Springer-Verlag, 1995.

[69] Umberto Straccia. A sequent calculus for reasoning in four-valued description logics. Didier Galmiche, editor, Proceedings of the 6th International Conference on Automated Reasoning with Analytic Tableaux and Related Methods (TABLEAUX 1997), volume 1227 of Lecture Notes in Computer Science, pages 343-357. Springer-Verlag, 1997

[70] Yue Ma Xiaowang Zhang, Guilin Qi and Zuoquan Lin. Quasi-classical semantics for expressive description logics. Bernardo Cuenca Grau, Ian Horrocks, Boris Motik, Ulrike Sattler, editors, Proceedings of the 22nd International Workshop on Description Logics (DL2009), Oxford, UK, July 27-30, 2009. CEUR Workshop Proceedings vol. 477. CEUR-WS.org, 2009.

[71] Chiaki Sakama and Katsumi Inoue. Paraconsistent stable semantics for extended disjunctive Programs. Journal of Logic and Computation, 5(3): 265-285, 1995.

[72] Chiaki Sakama. Extended well-founded semantics for paraconsistent logic programs, Proceedings of the International Conference on Fifth Generation Computer Systems, June 1-5, 1992, Tokyo, Japan, pages 592599. IOS Press, 1992.

[73] Anbu Yue and Zuoquan Lin. A coherent and paraconsistent variant of the default logic. Proceedings of the 8th International Symposium on Logical Formalizations of Commonsense Reasoning (COMMONSENSE 2007). Technical Report SS-07-05. AAAI Press, 2007.

[74] Yao-Hua Tan and Johan van den Akker. QML: a paraconsistent default logic. Logique \& Analyse 143-144: 311-328, 1993.

[75] Hai Wang, Matthew Horridge, Alan Rector, Nick Drummond, and Julian Seidenberg. Debugging OWLDL ontologies: A heuristic approach. Gil, Yolanda and Motta, Enrico and Benjamins, V. and Musen, Mark, editors, Proceedings of the 4 th International Semantic Web Conference (ISWC 2005), Galway, Ireland, November 6-10 2005. LNCS 3729, pages 745 757. Springer Berlin / Heidelberg, 2005. 